\author{
Aus der Abteilung für \\ Unfallchirurgie, Plastische und Wiederherstellungschirurgie \\ (Prof. Dr. med. K.M. Stürmer) \\ Im Zentrum Chirurgie \\ der Medizinischen Fakultät der Universität Göttingen
}

\title{
Klinische und biomechanische Untersuchungen zur operativen Versorgung von Mittelhandfrakturen mit resorbierbaren Implantaten
}

\author{
INAUGURAL - DISSERTATION \\ zur \\ Erlangung des Doktorgrades \\ der Medizinischen Fakultät der \\ Georg-August-Universität zu Göttingen
}

vorgelegt von

Daniel Appelt

aus München

Göttingen, 2011 
Dekan:

I. Berichterstatter:

II. Berichterstatter/in:

Tag der mündlichen Prüfung:
Prof. Dr. med. C. Frömmel

Priv.-Doz. Dr. med. C. Dumont

Priv.-Doz. Dr. med. dent. W. Hahn 06.07.2011 


\section{INHALTSVERZEICHNIS}

1 EINLEITUNG

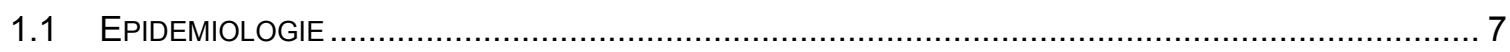

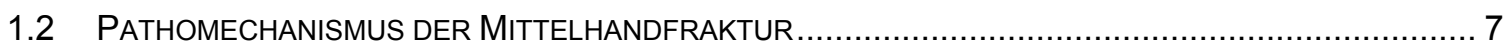

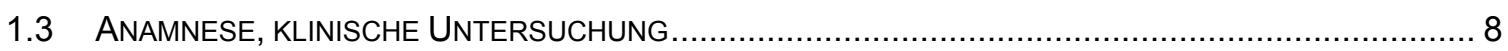

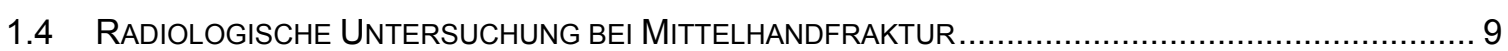

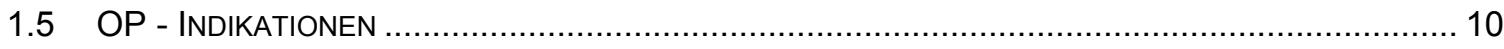

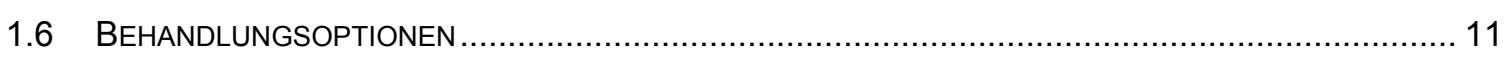

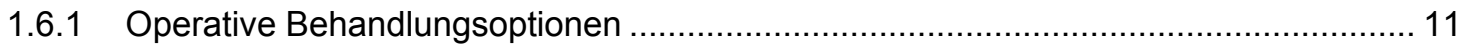

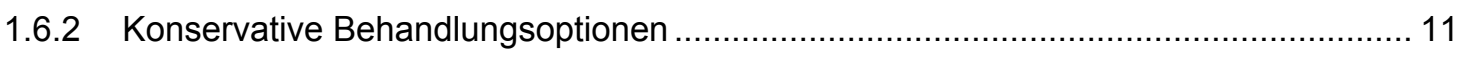

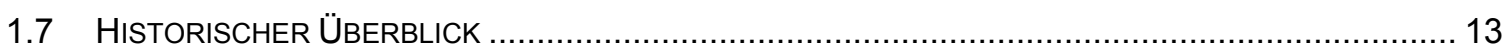

1.8 ENTWICKLUNG RESORBIERBARER OSTEOSYNTHESEMATERIALIEN ......................................... 14

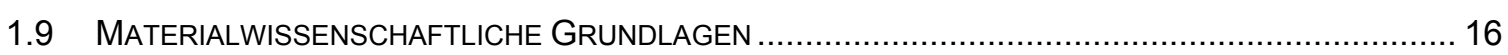

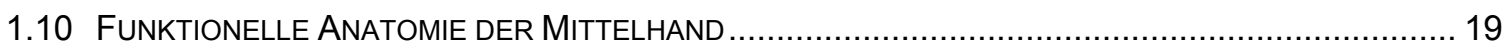

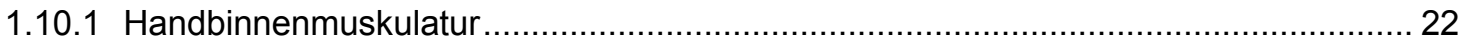

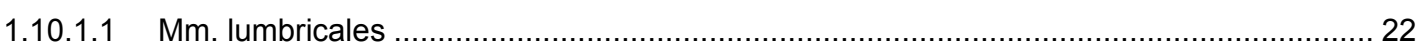

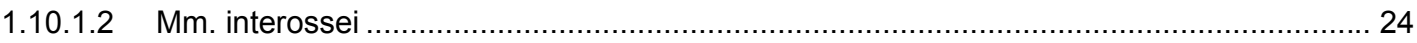

1.11 OP-TECHNIK (PLATTENOStEOSYNTHESE, RESORBIERBARES IMPLANTAT) …............................. 25

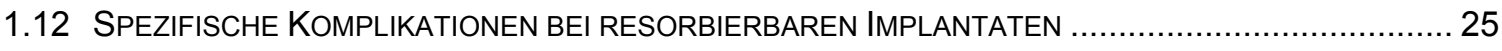

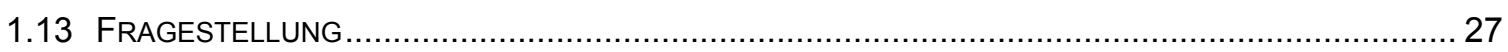

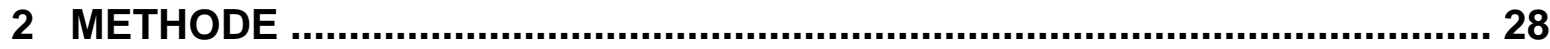

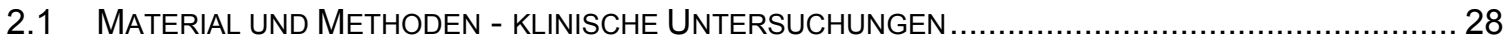

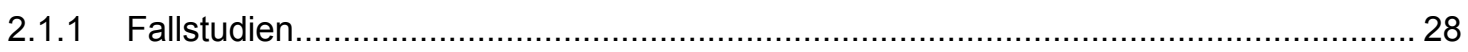

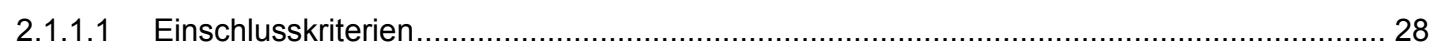

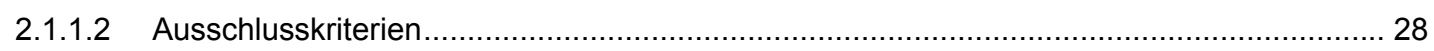

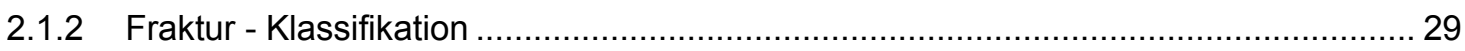

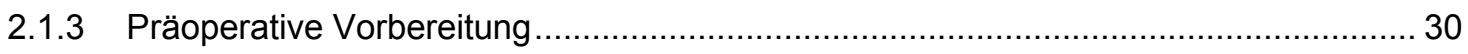

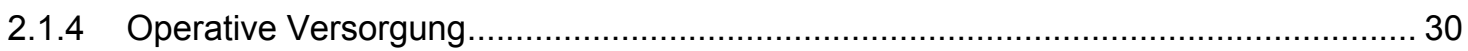

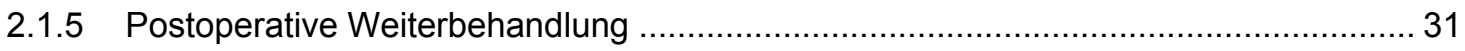

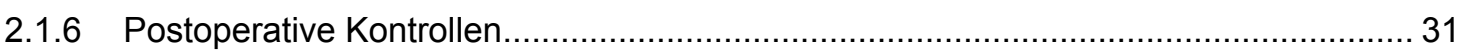

2.1.6.1 Radiologische Auswertungskriterien..................................................................... 31

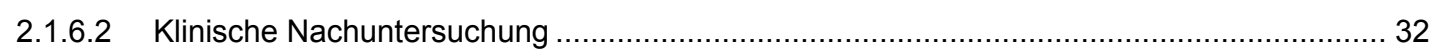

2.1.6.2.1 Visuelle Analogskala (VAS) und DASH - Score ........................................................ 33

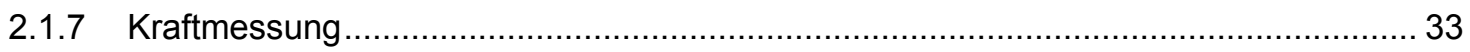

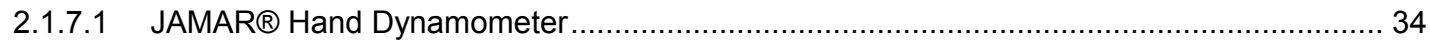

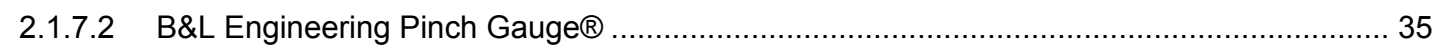

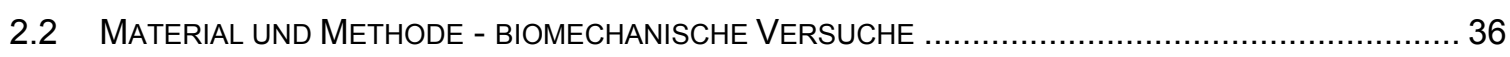

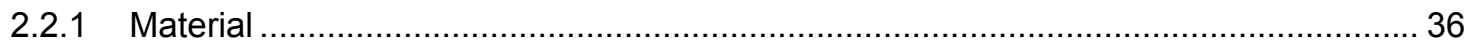

2.2.1.1 Tiermodell für humane Mittelhandknochen ............................................................. 36

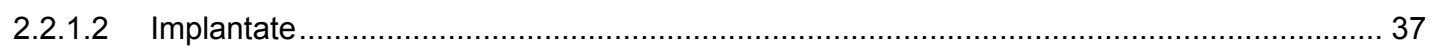




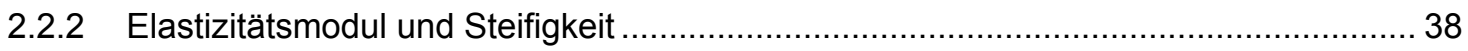

2.2.3 Zwick - Prüfmaschine und Einstellungen................................................................... 39

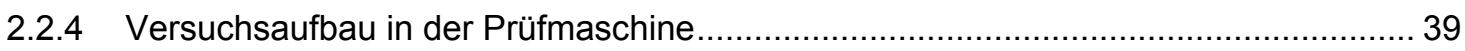

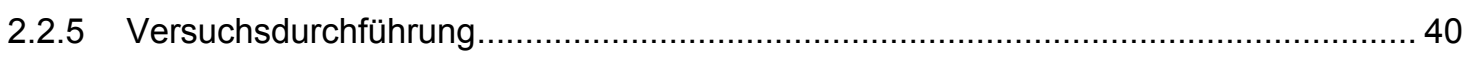

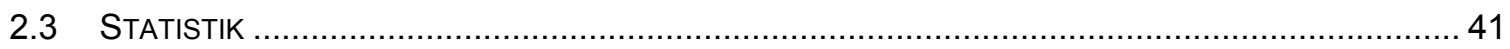

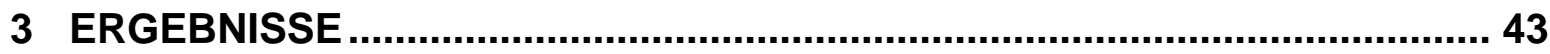

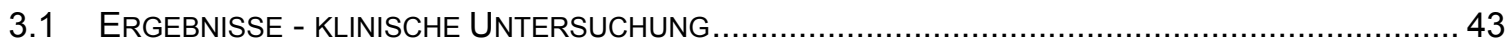

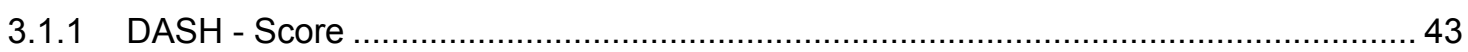

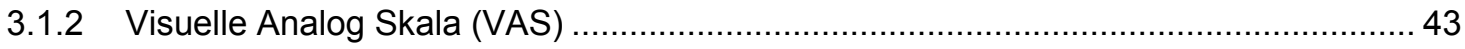

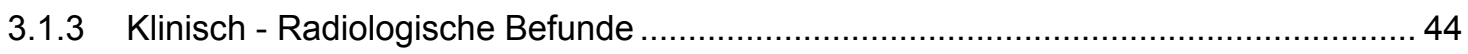

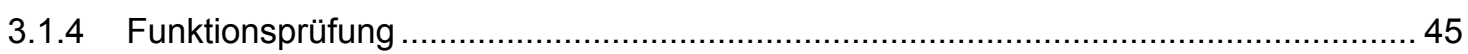

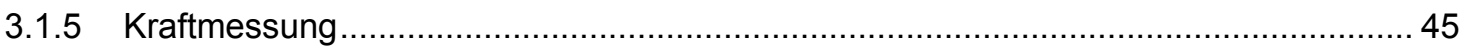

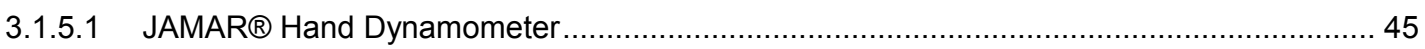

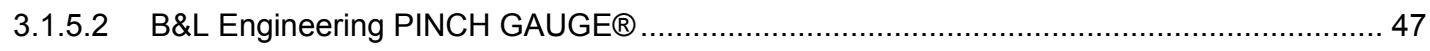

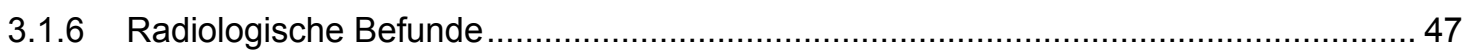

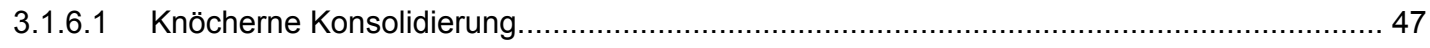

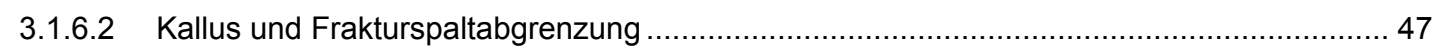

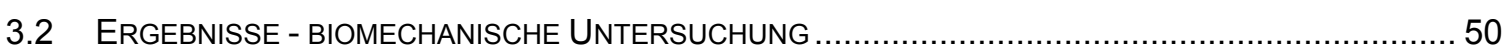

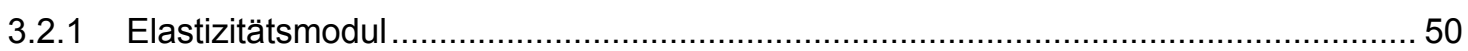

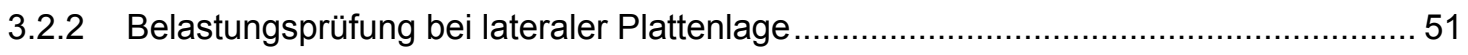

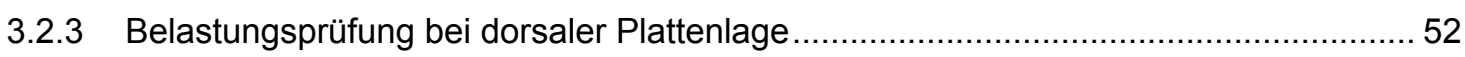

4 DISKUSSION

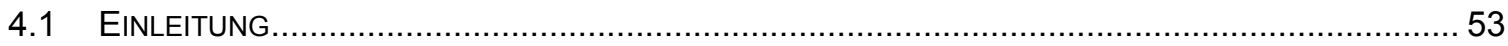

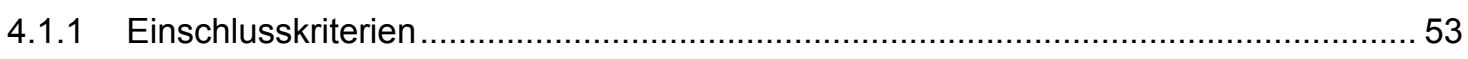

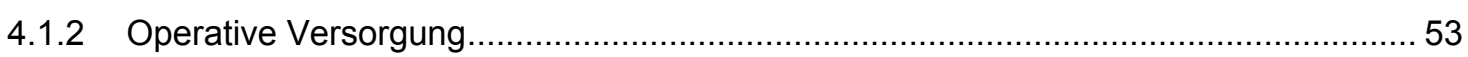

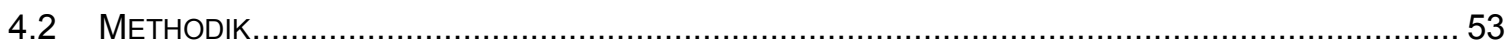

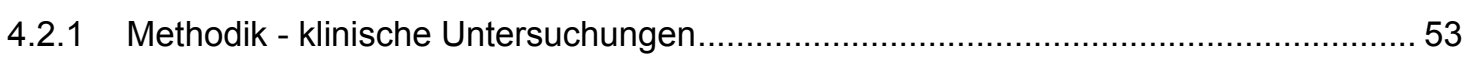

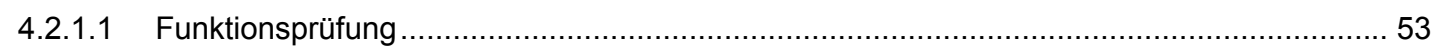

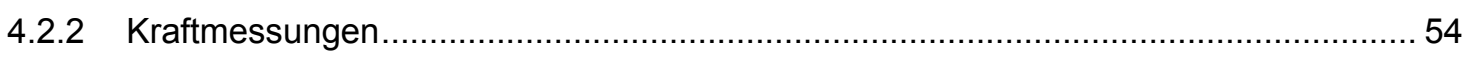

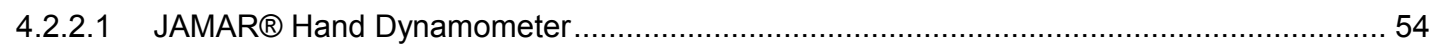

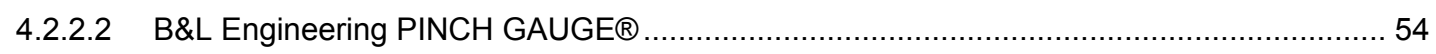

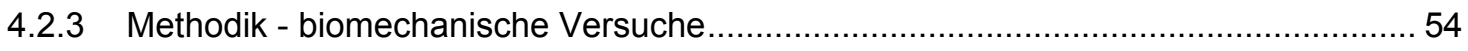

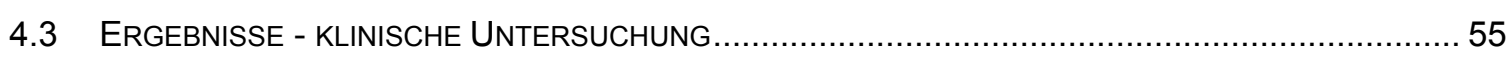

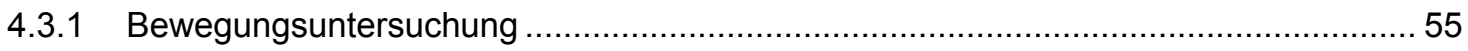

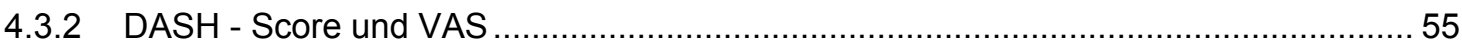

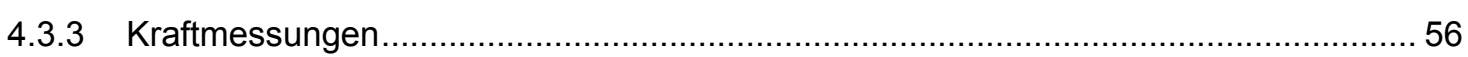

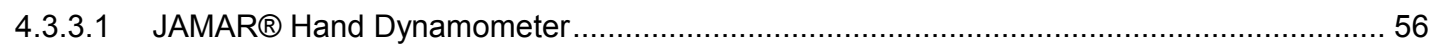

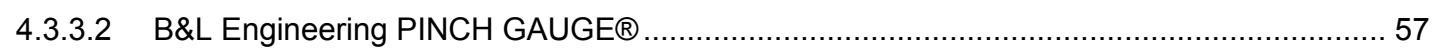

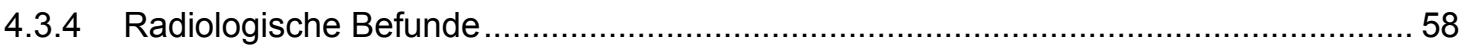

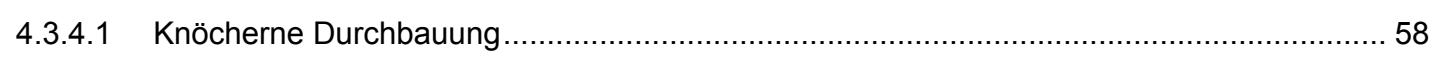

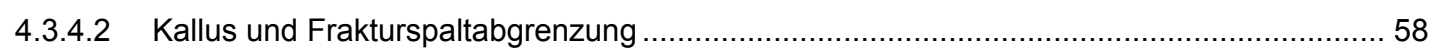




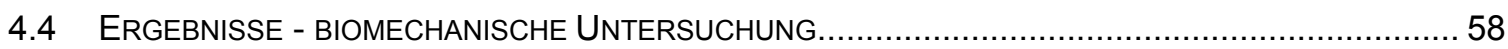

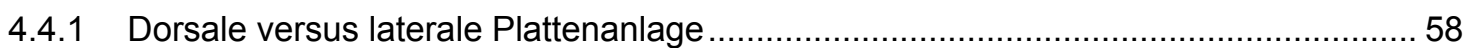

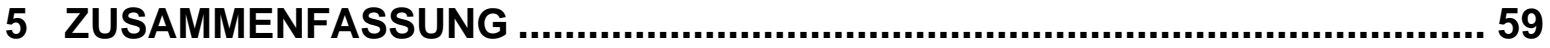

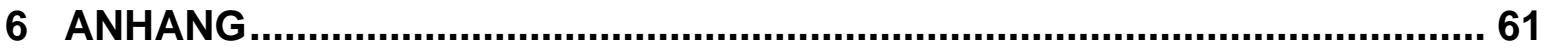

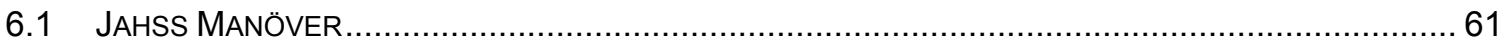

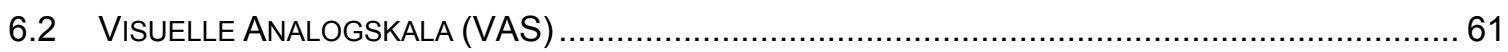

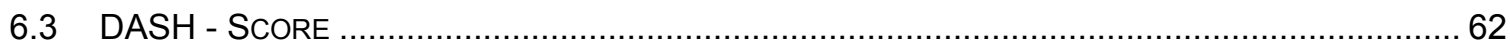

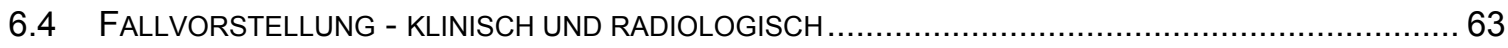

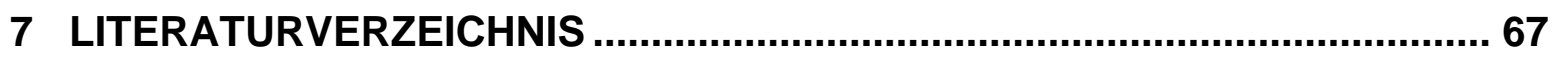

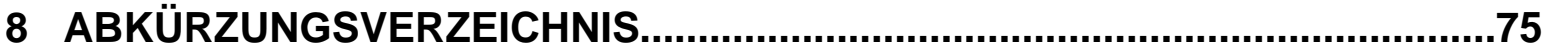




\section{Abbildungsverzeichnis}

ABB. 1:

ABB. 2:

ABB. 3:

ABB. 4:

ABB. 5:

ABB. 6:

ABB. 8:

ABB. 9:

ABB. 10:

ABB. 11:

ABB. 12:

ABB. 13:

ABB. 14:

ABB. 15:

ABB. 16:

ABB. 17:

ABB. 18:

ABB. 19:

ABB. 20:

ABB. 21:

ABB. 22:

ABB. 23:

ABB. 24 - 29:
L - MILCHSÄURE UND GLYKOLSÄURE MONOMERE 18

DREI BÖGEN DES HANDSKELETTS 20

KONVERGIERENDE FINGERACHSEN 21

LÄNGSCHNITTE DURCH HUMANE MITTELHANDKNOCHEN 22

MM. LUMBRICALES (PALMAR) 23

MM. INTEROSSEI PALMARES UND DORSALES 25

FALLBEISPIEL 1213

FALLBEISPIEL 2

ALPHA - NUMERISCHE KODIERUNG DES HANDSKELETTS 29

MITTELHANDORTHESE $\quad 31$

FUNKTIONSPRÜFUNGEN 32

JAMAR® HAND DYNAMOMETER $\quad 35$

B\&L ENGINEERING PINCH GAUGE® (60 LB) 35

ANATOMISCHE ZEICHNUNG EINES SCHWEINEVORDERFUßES 36

RÖNTGENAUFNAHME PORCINER MITTELFUßKNOCHEN 37

VERWENDETE IMPLANTATE $\quad 37$

3 - PUNKT - BIEGEVERSUCHSAUFBAU 39

QUERE MITTELSCHAFTOSTEOTOMIE $\quad 40$

INSTRUMENTARIUM $\quad 41$

DORSO - LATERALE PLATTENANLAGE $\quad 41$

JAHSS - MANÖVER $\quad 62$

VISUELLE ANALOGSKALA 61

$\begin{array}{ll}\text { FALLBEISPIELE } & 64-66\end{array}$ 


\section{Einleitung}

\subsection{Epidemiologie}

Mittelhandfrakturen sind häufige Verletzungen der oberen Extremität, die besonders junge Menschen betreffen (Abdon et al. 1984, Crosby et al. 1994, Prevel et al. 1995, Prevel et al. 1996, Prokop et al. 1999, Prokop et al. 2002, Schaefer und Siebert 2000). Frakturen der Mittelhand und Fingerknochen machen ca. 10\% aller Frakturen aus. Die Randstrahlen, die funktionelle Einheit aus Finger- und Mittelhandknochen, sind häufiger von Frakturen betroffen als die zentralen Strahlen (Barton 1984, De Jonge et al. 1994, Schaefer und Siebert 2000).

\subsection{Pathomechanismus der Mittelhandfraktur}

Häufig entstehen die Mittelhandfrakturen durch Sturz oder Quetschung. In 50\% der Fälle handelt es sich um die Folgen eines Arbeitsunfalls. Bei subcapitalen Mittelhandfrakturen spielen axiale Traumen eine wichtige Rolle (Boxer - Fraktur) (Freeland und Jabaley 1998, Freeland und Orbay 2006, Schmidt und Lanz 1992). Durch Sturz auf die dorsalflektierte Hand kommt es zu Basisfrakturen der Mittelhandknochen, eventuell als Luxationsfraktur (Schaefer und Siebert 2000, Taeger et al. 2007).

Die häufigsten Frakturen der Mittelhand sind die subcapitalen Frakturen am V., gefolgt vom IV. Mittelhandknochen, die dritthäufigsten sind die basisnahen des I. Mittelhandknochens (Schaefer und Siebert 2000, Strauch et al. 1998).

Die Dislokation einer basisnahen Fraktur des IV. MHK von mehr als $50^{\circ}$, des V. Mittelhandknochens von mehr als $70^{\circ}$, gehen häufig mit Begleitverletzungen der benachbarten Mittelhandknochen in Form von Fraktur, Dislokation oder Luxation einher (Strauch et al. 1998).

Mittelhandfrakturen können in drei Ebenen dislozieren: Rotation um die Längsachse, Abkippung in der Frontal- und Sagittalebene, Verkürzung entlang der Längsachse (Schaefer und Siebert 2000, Strauch et al. 1998). Die Mittelhandfrakturen des II. und V. Strahls neigen zu starker Dislokation, da sie nur einseitig von intermetacarpalen Ligamenten unterstützt werden. Die Mittelhandknochen des III. und IV. Strahls erfahren eine beidseitige Unterstützung durch die intermetacarpalen Ligamente, und sind somit gegen eine Dislokation von mehr als 3 - $4 \mathrm{~mm}$ gesichert, insbesondere bei distalen und diaphysären Frakturen (Freeland und Jabaley 1998, 
Freeland und Orbay 2006, Freeland et al. 2001). Biomechanische Studien an Leichenhänden haben gezeigt, dass Dislokationen von mehr als $30^{\circ}$ und Verkürzungen von mehr als 3 - $4 \mathrm{~mm}$ zu einer signifikanten Veränderung des Kräftegleichgewichts zwischen Extensoren und Flexoren der Hand führen (Low et al. 1995).

Birndorf zeigte, daß sich ab einer palmaren Dislokation von $30^{\circ}$ die Flexorsehnen relativ um $4 \mathrm{~mm}$ verlängerten. Als Folge daraus stieg die durchschnittliche mechanische Arbeit ab diesem Dislokationsgrad signifikant an (Birndorf et al. 1997).

Strauch eruierte an 9 Leichenhänden ein durchschnittliches Extensionsdefizit von $7^{\circ}$ je $2 \mathrm{~mm}$ Verkürzung am MHK II und $\mathrm{V}$. Die MHK II bis $\mathrm{V}$ wiesen dabei keine funktionellen Unterschiede auf (Birndorf et al. 1997, Low et al. 1995, Strauch et al. 1998).

\subsection{Anamnese, klinische Untersuchung}

Das gesamte Ausmaß der Handverletzung, insbesondere des Weichteilmantels, kann häufig in den ersten Stunden nach dem Unfall nicht erkannt werden, weshalb eine exakte Befragung nach der Unfallursache und dem Mechanismus für Prognose und Behandlung von eminenter Bedeutung sind.

Die klinische Untersuchung umfasst Haut, Muskel, Sehnen, Gelenke, Bänder, Nerven und Durchblutung. Schmerzen, Bewegungseinschränkungen, Schwellung sowie Krepitation im verletzten Gebiet können neben Achsabweichungen einen ersten Hinweis auf eine Fraktur geben. Hautabschürfungen und Quetschmarken weisen ebenfalls auf eine Verletzung hin.

Zeigt sich bei Inspektion der Rückseite der Finger in Beugung und Streckung eine veränderte Stellung der Fingernägel, so kann das ein Hinweis auf Vorliegen einer Mittelhandfraktur sein. Tritt durch eine Fraktur eine Rotationsabweichung auf, steht am entsprechenden Finger die Nageloberfläche nicht mehr in einer Ebene mit den anderen Fingern, sondern leicht gedreht (Royle 1990). Bei Palpation der betroffenen Handregion lässt sich ein Druckschmerz auslösen. Funktionell zentrieren sich die Fingerspitzen beim halben Faustschluss bei einer Rotationsfehlstellung nicht auf einen Punkt über dem Os scaphoideum bzw. Os lunatum (Abbildung 5), sondern der betroffene Fingerstrahl - oder bei Serienfrakturen mehrere - zeigt in eine andere Richtung. Je weiter proximal der Mittelhandknochen frakturiert ist, desto mehr wirkt sich eine Rotationsabweichung aus (Schmidt und Lanz 1992). 


\subsection{Radiologische Untersuchung bei Mittelhandfraktur}

Frakturen der Mittelhand werden mit Röntgenaufnahmen in mindestens zwei Ebenen (dorsopalmarer und schräger Strahlengang) nachgewiesen. Häufig wird eine dritte Aufnahme im streng seitlichen Strahlengang zum Ausschluss karpometakarpaler Luxationen benötigt (Tavassoli et al. 2005). Bei der Darstellung des II. und III. Strahls wird die Schrägaufnahme in $45^{\circ}$ - Pronationsstellung mit Zentrierung auf die Basis von MHK II angefertigt. Bei Darstellung der Strahlen IV und V in $45^{\circ}$ - Supinationsstellung (Zitherspieler-Projektion, Abbildung 1) mit Zentrierung auf die Basis von MHK IV, (Arbeitskreis "bildgebende Diagnostik in der Rheumatologie“ 1997).

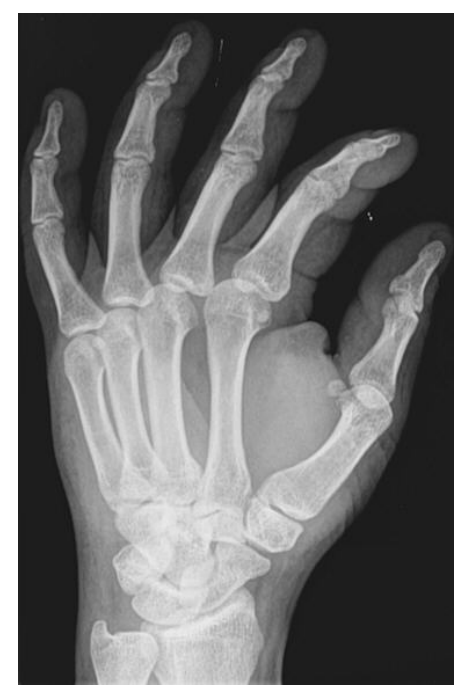

Abbildung 1: Zitherspieler-Projektion

Nur bei dislozierten Frakturen der Mittelhandknochenbasis und des Mittelhandknochenkopfes ist gelegentlich eine weiterführende Diagnostik (CT, MRT) erforderlich. Zur besonderen Beurteilung der MCP - Gelenke und Identifikation von dislozierten Avulsionsfrakturen dient eine Spezialaufnahme nach Brewerton (Brewerton 1967, Lourie et al. 2006). Hierbei kann der grubenförmige Übergang an den Außenseiten des Mittelhandknochenkopfes zum distalen Schaftabschnitt gut beurteilt werden (Schaefer und Siebert 2000, Strauch et al. 1998).

Für die radiologische Beurteilung des Dislokationsausmaßes im konventionellen Röntgenbild gelten folgende Regeln: Die Verkürzung eines Mittelhandknochens wird anhand der Verbindungslinie entlang der Mittelhandknochenköpfe III - V beurteilt (sog. "Metakarpalzeichen“). Normalerweise verbindet diese Linie die Mittelhandknochen III - V, während sich das Mittelhand - Köpfchen II deutlich unter dieser Geraden befindet. 


\subsection{OP - Indikationen}

Von 1997 - 1999 führte die Handgruppe der Deutschen Sektion der AO International eine multizentrische Studie zum Thema „Frakturen der Mittelhand - Indikationen und Behandlungsoptionen" durch (Prokop et al. 1999). Aus dieser Studie ergaben sich die Richtlinien zur operativen Behandlung von Mittelhandfrakturen.

Bereits eine Verkürzung durch Einstauchung einer Mittelhandfraktur von $2 \mathrm{~mm}$ führt zu einem Streckdefizit von ca. $7^{\circ}$ (Freeland und Jabaley 1998, Freeland und Orbay 2006, Freeland et al. 2001, Hughes 2006, Low et al. 1995, Strauch et al. 1998). Eine Abweichung von mehr als $30^{\circ}$ sowie eine Einstauchung von mehr als $4 \mathrm{~mm}$ führen zu einer Störung der intrinsischen Muskel - Sehnen - Dynamik. Dies kann zu muskulärer Schwäche, Verlust der Ausdauer, Krämpfen und der „Klauenhand“ führen (Strauch et al. 1998).

Rotationsabweichungen der Mittelhandknochen können zu einer Störung des Faustschlusses führen. Eine Rotationsabweichung von $5^{\circ}$ wirkt sich als Überlappung der Finger von 1,5 cm bei Flexion aus (Freeland und Jabaley 1998, Freeland und Orbay 2006, Freeland et al. 2001, Hughes 2006).

Folgende Frakturtypen sollten operativ stabilisiert werden (Küntscher et al. 2003, Larkin et al. 1997, Prokop et al. 1999):

- Mehrfragment- und Trümmerfrakturen

- Serienfrakturen

- Intraartikuläre Frakturen

- Dislozierte Frakturen:

o Rotationsabweichung

o mehr als $10^{\circ}$ laterale oder dorsale Dislokation

o mehr als $2 \mathrm{~mm}$ Verkürzung

o mehr als $30^{\circ}$ palmare Dislokation für distale Frakturen des 4 . und 5 . MHK

o mehr als $20^{\circ}$ palmare Dislokation für MHK - Schaftfrakturen

- Offene Frakturen

- Versagen der konservativen Therapie 


\subsection{Behandlungsoptionen}

\subsubsection{Operative Behandlungsoptionen}

Folgende Formen der Reposition bzw. Osteosynthese haben sich für die einzelnen Frakturlokalisationen bewährt (Küntscher et al. 2003, Petracic und Siebert 1998, Pietrzak et al. 1996, Pietrzak et al. 1997, Prevel et al. 1995, Prevel et al. 1996, Prokop et al. 2002, Prokop et al. 1999):

o Diaphysäre Frakturen des 2. - 5. MHK

0 perkutane antegrade intramedulläre K-Draht-Osteosynthese

o offene Reposition und Platten- bzw. Schraubenosteosynthese

o Distale Frakturen des 2. - 5. MHK

o Metaphysär: antegrade intramedulläre K-Draht-Osteosynthese

o Intraartikulär: offene Reposition und Platten bzw. Schraubenosteosynthese geschlossene Reposition und KDraht-Osteosynthese

o Proximale, basisnahe Frakturen des 2. - 5. MHK:

o Metaphysär: offene Reposition (Platten- bzw. Schraubenosteosynthese) oder geschlossene Reposition und K-DrahtOsteosynthese

o Intraartikulär: offene Reposition (Platten- bzw. Schraubenosteosynthese).

\subsubsection{Konservative Behandlungsoptionen}

Bain und Guy stellten 2003 eine Tape - Verbandtechnik als konservative Behandlungsmethode vor. Hierbei wurde unter Bruchspaltanästhesie die Reposition der Fraktur mit Hilfe des Jahss - Manövers durchgeführt (Anhang 6.1). Um das Repositionsergebnis zu sichern, wurde der Finger des frakturierten MHK mit dem Nachbarfinger mit Tape verbunden, sogenanntes Buddy - Taping. Durch die Anlage des Tapes in einer Zugrichtung von radial über palmar nach ulnar konnte der entstehende Pronationsstress die Frakturenden in Position halten. Die Weiterbehandlung erfolgte für eine Woche in einer Schiene; der Tape - Verband wurde für weitere drei Wochen beibehalten. Nach insgesamt vier Wochen durften die Patienten wieder an Kontaktsportarten teilnehmen (Bain und Guy 2003). 
Tavassoli et al. benutzen drei verschiedene Gipstechniken, um Mittelhandfrakturen konservativ zu behandeln. Je eine Technik wurde von je einem Chirurgen unabhängig vom Frakturtyp angewandt (Tavassoli et al. 2005). Bei der 1. Gips - Technik wurde das MCP - Gelenk des betroffenen Strahls in Flexion immobilisert, die IP Gelenke blieben frei. Dadurch wurde Traktion nur auf den frakturierten MHK ausgeübt.

In der 2. Variante wurden alle MCP - Gelenke in Extension eingegipst. Die IP Gelenke blieben ebenfalls frei.

Als 3. Version wurde das MCP - Gelenk in Flexion eingegipst und die IP - Gelenke in Extension immobilisiert. Es wurde keine wesentliche Zunahme der Rotationsabweichung $\left(3^{\circ}\right.$, Standardabweichung $\left.4^{\circ}\right)$ beobachtet. Neun Wochen nach Reposition wurde der Gips entfernt. Im Verglich zur gesunden Gegenseite betrug die Griffstärke 85 - 90\%. Der Bewegungsumfang erreichte 82 - 89\% (Tavassoli et al. 2005).

Prokop et al. fassten 2002 die Behandlungsmaßnahmen bei Mittelhandfrakturen zusammen: Mittelhandfrakturen werden primär konservativ behandelt, wenn keine Gelenkstufen, Achsabweichungen über $30 \mathrm{Grad}$, Rotationsfehlstellungen, Verkürzungen von über $5 \mathrm{~mm}$, Serienfrakturen oder offene Frakturen bestehen. Stabile, nicht dislozierte Frakturen sollten frühfunktionell behandelt werden (Prokop et al. 2002). Buddy - Tapes für drei bis vier Wochen verhindern eine Rotationsfehlstellung und ermöglichen eine funktionelle Nachbehandlung (Küntscher et al. 2003, Schaefer und Siebert 2000).

Geschlossene Repositionen der am häufigsten beobachteten dislozierten subkapitalen Boxerfrakturen am 5. Mittelhandknochen führen nicht zu einem befriedigenden Ergebnis. Bei Dislokationen über $30 \mathrm{Grad}$ nach palmar sollte eine übungsstabile intramedulläre Schienung erfolgen (Prokop et al. 1999, Prokop et al. 2002).

An einem Patientenbeispiel lässt sich der Einfluss eines guten Operationsergebnisses verdeutlichen: Der Patient hatte sich die linksseitigen MHK III und IV gebrochen und wurde im Anschluss operativ mittels resorbierbarer Plattenosteosynthese versorgt. Das klinische Ergebnis ist sehr zufriedenstellend. Ca. 11/2 Jahre zuvor hatte sich der Patient den rechtsseitigen MHK V gebrochen, wurde aber nicht operiert. Nach Ausheilung der rechtsseitigen MHK $\vee$ Fraktur blieb eine Rotationsabweichung zurück, die sich in einer Überlappung der Fingerendglieder manifestiert (Abbildung 2). 


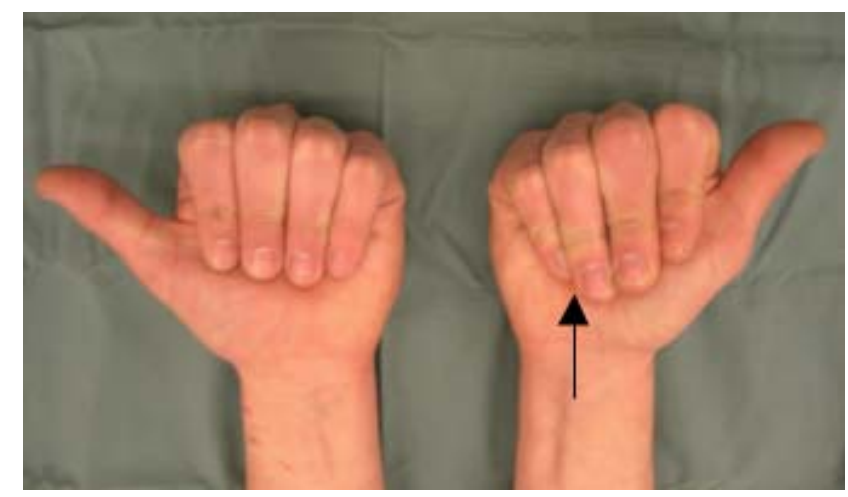

Abbildung 2: Fallbeispiel 1: Im Seitenvergleich zeigt sich eine leichte Überlappung des Ring - und Kleinfingers der rechten Hand, hervorgerufen durch die Rotationsabweichung nach konservativer Therapie bei MHK V - Fraktur rechts

\subsection{Historischer Überblick}

Das Prinzip von Osteosynthesen an der Hand besteht darin, dislozierte oder instabile Frakturen zu reponieren und zu retenieren (Freeland und Jabaley 1998, Freeland und Orbay 2006, Freeland et al. 2001, Müller et al. 1969, Stoffel et al. 2000). Angestrebt wird die achsen -, längen - und rotationsgerechte Frakturreposition und - retention, die eine aktive Frühmobilisierung der Finger und Hände während der Frakturheilung erlaubt (Duncan et al. 1993, Freeland und Jabaley 1998, Freeland und Orbay 2006, Freeland et al. 2001, Müller et al. 1969, Stoffel et al. 2000). Der Leitspruch „function follows form" spiegelt dieses Prinzip wieder, denn die Funktion der Hand hängt wesentlich von der Anatomie des Handskeletts ab (Freeland und Jabaley 1998, Freeland und Orbay 2006, Freeland et al. 2001). Achsabweichungen oder Rotationsabweichungen eines frakturierten Strahls behindern ab einem gewissen Ausmaß die Funktion der benachbarten Strahlen und damit der Hand (Freeland und Jabaley 1998, Freeland und Orbay 2006, Freeland et al. 2001). Die Verkürzung eines Knochens beeinflusst die Muskel - Sehnen - Spannung, was zur Einschränkung des Bewegungsumfangs und der Kraft der Finger führt (Freeland und Jabaley 1998, Freeland und Orbay 2006, Freeland et al. 2001, Low et al. 1995).

Zusammenfassend werden als Ziel bei der Frakturbehandlung die achs -, rotations - und längengerechte Repostion und wenn möglich, übungsstabile Retention einer Fraktur angestrebt (Müller et al. 1969). 


\subsection{Entwicklung resorbierbarer Osteosynthesematerialien}

In der Unfallchirurgie sind Osteosynthesen mit Metallplatten und Schrauben aus Titan als bioinertes Material standardisiert und etabliert (Trevisan 2004, van der Elst 2000, van der Elst et al. 1998, van der Elst et al. 2000). Häufig erfordert das Verfahren jedoch die Materialentfernung in einem zweiten chirurgischen Eingriff, der eine körperliche, psychische und bisweilen auch finanzielle Belastung für die Patienten darstellt (van der Elst 1998). Bei Belassen des Osteosynthesematerials nach Ausheilung der Fraktur bleibt es ohne Funktion und kann die umliegenden Weichteile beeinflussen (Hofmann 1992, van der Elst 2000, van der Elst et al. 1998, van der Elst et al. 2000).

Bei osteosynthetisch versorgten Mittelhandfrakturen durch eine dorsale Plattenanlage wurde die unmittelbare Nähe zu den Extensorsehnen als Quelle von Irritationen identifiziert (Bozic et al. 2001, Hughes 2006). In einigen Fällen sind Sehnenrupturen beschrieben (Bozic et al. 2001, Hughes 2006, van der Elst 2000, van der Elst et al. 1998, van der Elst et al. 2000).

Seit Anfang der 70er Jahre werden resorbierbare Osteosynthesematerialien entwickelt und erforscht (Bozic et al. 2001). Die resorbierbaren Materialien, welche schrittweise an Stabilität verlieren, nachdem sie implantiert worden sind, bieten möglicherweise das Potential für neue Wege in der osteosynthetischen Versorgung (Bozic et al. 2001). Erste Erfahrungen wurden tierexperimentell mit synthetischen biodegradierbaren Nahtmaterialien gewonnen (Hofmann 1992, Hofmann 1997, Hofmann et al. 1990, Hofmann und Wagner 1993, Hofmann et al. 1996).

Es wird angenommen, dass durch den schrittweisen Abbau des resorbierbaren Osteosynthesematerials weniger "stress shielding" entsteht, wobei die Extistenz des "stress shieldings“ in der Literatur widersprüchlich diskutiert wird (Hughes 2006, Stoffel et al. 2000).

Bozic et al. und Hughes schlagen vor, daß Metallimplantate der Festigkeit und Steifigkeit von Knochen entsprechen sollen. Dadurch werden direkt nach der Implantation die Beweglichkeit der angrenzenden Gelenke und vor allem die Belastbarkeit des frakturierten Knochens erreicht (Bozic et al. 2001, Hughes 2006). Während der Entwicklung der Osteosyntheseimplantate hat sich gezeigt, dass die Knochenheilung durch zu steife Implantate negativ beeinflusst wird (Stürmer et al. 1996).

In der biomechanischen Studie von Bozic et al. wurde eine standardisierte diaphysäre Osteotomie am synthetisch hergestellten Modell eines humanen Mittelhand- 
knochens mit Titanplatten oder bioresorbierbaren Platten versorgt und zu unterschiedlichen Zeitpunkten einem 4 - Punkt - Biegeversuch unterzogen. Um den in vivo - Degradationsprozess zu simulieren, wurden die Knochen in der Zeit zwischen Osteosynthese und Versuch in erwärmter Salzlösung gelagert (Bozic et al. 2001). Als Ergebnis dieser Studie lässt sich festhalten, daß die bioresorbierbaren Implantate eine konstante Festigkeit und Steifigkeit bis zum Untersuchungszeitpunkt 8 Wochen nach Osteosynthese aufwiesen. Dies entspricht dem Zeitraum, der in der Regel notwendig ist, um die knöcherne Konsolidierung zu erreichen (Bozic et al. 2001, Pietrzak et al. 1996, Pietrzak et al. 1997). Die Wahrscheinlichkeit der Irritation der Extensorsehnen wird deutlich reduziert (Bozic et al. 2001).

\section{Dynamik der Knochenheilung und Abnahme der Lactosorb - Festigkeit}

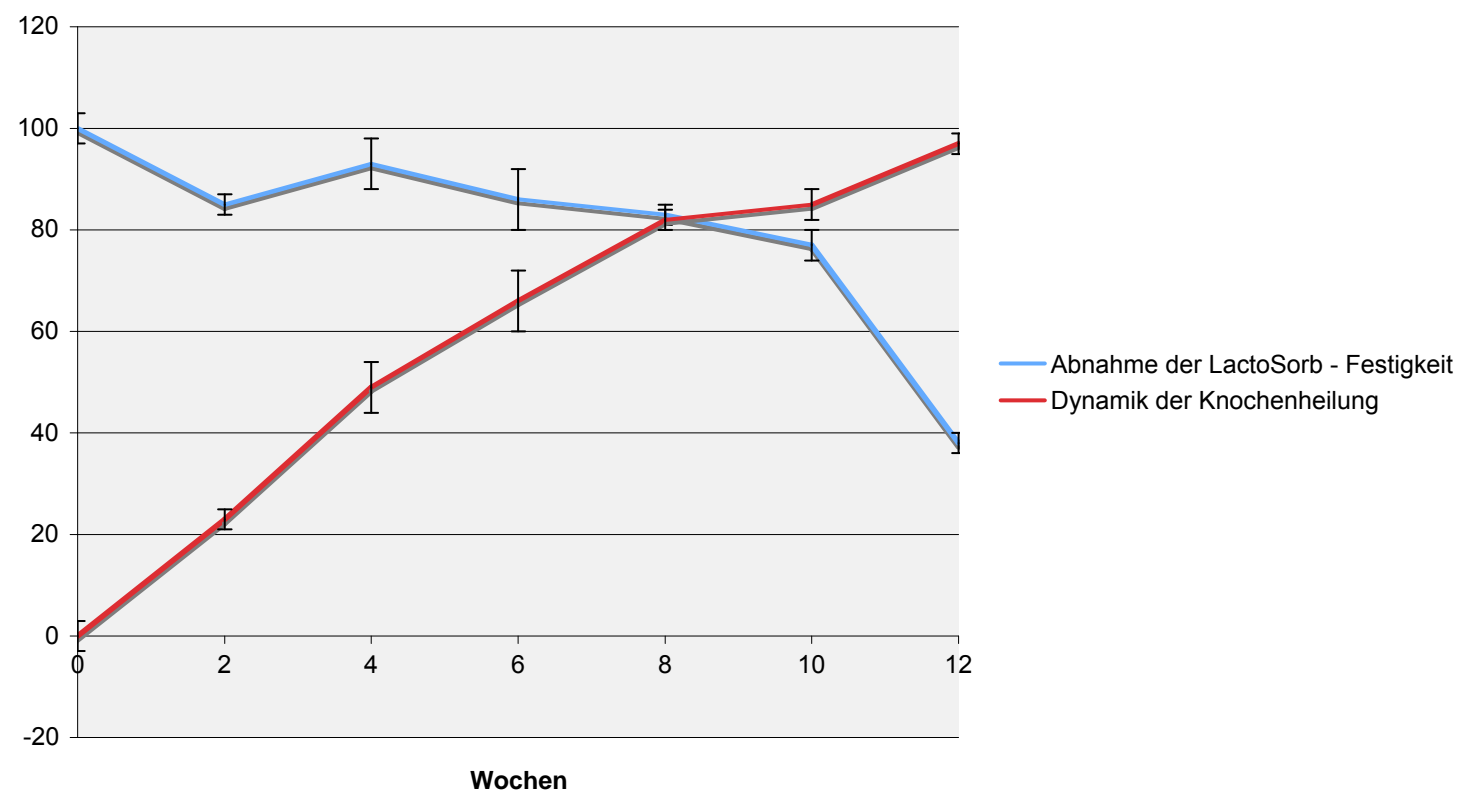

Diagramm 1: modifiziert nach Bozic et al. 2001 und Pietrzak et al. 1996 und 1997

Verschiedene Probleme haben die Verbreitung von biodegradierbaren Implantaten behindert. Bei Implantaten, die aus Polyglykolsäure (PGA) bestehen, war der in vivo Abbau zu schnell vorangeschritten, sodass Refrakturen aufgetreten waren (Waris et al. 2002, Waris et al. 2004). Andererseits wurden sogar noch nach 5 Jahren Bestandteile des ursprünglichen Implantats gefunden (Bozic et al. 2001, Navarro et al. 2008, Waris et al. 2002, Waris et al. 2004). Dies führte zu der Frage, ob PGA - Implantate nicht zu „biostabil“ seien, um in der modernen Chirurgie Ver- 
wendung finden zu können (Bozic et al. 2001, Hofmann 1992, Hofmann 1997, Hofmann et al. 1990, Hofmann und Wagner 1993, Hofmann et al. 1996, Waris et al. 2002, Waris et al. 2004, Weiler 1996). Darüber hinaus waren bei der Degradation von bioresorbierbaren Implantaten so genannte "sterile Entzündungen“ aufgetreten (siehe auch: Kapitel 1.12 Komplikationen bei resorbierbaren Implantaten). Einige Theorien sehen die mangelnde Resorptionsfähigkeit des umgebenden bradytrophen Gewebes als Ursache solcher Reaktionen (Hofmann 1992, Hofmann 1997, Hofmann et al. 1990, Hofmann und Wagner 1993, Hofmann et al. 1996). Befürchtungen, unter resorbierbaren Implantaten käme es zu verstärkten Frakturspaltbewegungen und einer erhöhten Inzidenz von Pseudarthrosen, wurde mit der Entwicklung von „self - reinforced“ Materialien begegnet (Hughes 2006, Nunley und Joneschild 2006, Navarro et al. 2008). Diese „selbst - wiederverstärkten“ Implantate bestehen aus den monomeren Grundeinheiten Milchsäure und Glykolsäure (Abbildung 2). Die strukturelle Rigidität und Stabilität der „self - reinforced“ Implantate beruht auf der Ausbildung dreidimensionaler Netzwerke der monomeren Einheiten, worauf im folgenden Kapitel näher eingegangen wird (Hofmann 1992, Hofmann 1997, Hofmann und Wagner 1993, Hofmann et al. 1990, Hofmann et al. 1996, Navarro et al. 2008).

\subsection{Materialwissenschaftliche Grundlagen}

Jedes Polymer weist amorphe und kristalline Mikrostrukturen auf (Petracic und Siebert 1998, Pietrzak et al. 1996, Pietrzak 1997, van der Elst 2000, van der Elst et al. 1998, van der Elst et al. 2000).

Die Mikrostruktur eines Polymers entscheidet, ob es amorph oder kristallin ist (Petracic und Siebert 1998, Pietrzak et al. 1996, Pietrzak 1997, van der Elst 2000, van der Elst et al. 1998, van der Elst et al. 2000). In einer amorphen Mikrostruktur sind die einzelnen Polymerketten zufällig angeordnet. Sie sind leicht gegeneinander verschiebbar. Kristalline Strukturen sind geordnete Zustände, in denen benachbarte Polymerketten parallel und mit sehr kleinem Abstand angeordnet sind (Petracic und Siebert 1998, Pietrzak et al. 1996, Pietrzak 1997, van der Elst 2000, van der Elst et al. 1998, van der Elst et al. 2000). Kristalline Polymere haben eine hohe Dichte und sind belastbarer als amorphe Polymere. 
Alle Polymere besitzen eine charakteristische „Erweichungstemperatur", unter welcher sie rigide und verwindungssteif und über welcher sie thermoplastisch sind (Petracic und Siebert 1998, Pietrzak et al. 1996, Pietrzak 1997, van der Elst 2000 , van der Elst et al. 1998, van der Elst et al. 2000). 
Die folgenden Polymere sind bis heute erforscht (Barth, Navarro et al. 2008):

- Polyesteramide,

- Poly - beta - Hydroxybutyrate,

- Poly - beta - Hydroxypropionate, die sich in Versuchen jedoch nicht für eine Frakturversorgung eigneten

- Polydiaxanone (PDS), die jedoch wegen fehlender Festigkeit keine Verwendung in der Frakturversorgung finden,

- Polyglykolide (Dexane, Vicryl), diese zeigten zum Teil osteolytische Veränderungen am Knochen, und die

- Milchsäurecopolymere, die mit Glykolsäure dreidimensionale Strukturen bilden (Abbildung 3).<smiles>C[C@H](O)C(=O)O</smiles>

L-Milchsäure<smiles>O=C(O)CO</smiles>

Glycolsäure

Abbildung 3: L - Milchsäure und Glykolsäure als Monomere, wie sie im Lactosorb® - Copolymer vorkommen (zur Verfügung gestellt von der Fa. Biomet - Merck). 
Der Copolymerabbau verläuft unter Hydrolyse, wobei 2-Hydroxypropionat, das Salz der $\mathrm{L}$ - Milchsäure entsteht. Dieser Abbau geschieht in vier Schritten über die Hydratation mit der Auflösung der Wasserstoffbrückenbindungen, über den Festigkeitsverlust, den Formverlust durch Spaltung kovalenter Bindungen bis zum Masseverlust durch Auflösung niedermolekularer Verbindungen und Phagozytose kleinster Fragmente. Biochemisch wird L - Lactat über den Citratzyklus zu Adenosintriphosphat (ATP) und $\mathrm{CO}_{2}$ abgebaut (Petracic und Siebert 1998, Pietrzak et al. 1996, Pietrzak 1997).

\subsection{Funktionelle Anatomie der Mittelhand}

In der Mittelhand sind die fünf Strahlen der Hand ausgebildet, die sich zum Daumen und den vier Langfingern fortsetzen. Die Hand besteht aus 27 Knochen, davon 19 Röhrenknochen: 5 Mittelhandknochen und 14 Fingerknochen. Diese bilden 17 Gelenke. Jeder Fingerstrahl bildet eine polyartikuläre Kette, die aus einem Mittelhandknochen und drei Fingerknochen besteht. Der Daumen besteht aus zwei Fingerknochen (Schmidt und Lanz 1992).

Die Hand weist 3 funktionelle Bögen auf (Abbildung 4): einen queren Mittelhandbogen (a), der sich auf die Metacarpophalangealgelenke (MCP - Gelenke) projiziert, einen queren karpalen Bogen (b), sowie einen auf den 3. Strahl zentrierten Längsbogen (c) (Kapandji 1980). Der Längsbogen wird durch die dorsale Wölbung der Mittelhandknochen gebildet, die biomechanisch die Greiffunktion unterstützt (Schmidt und Lanz 1992). Der quere Mittelhandbogen ist durch die hohe Beweglichkeit an die Erfordernisse des Greifens anpassbar (Kapandji 1980). Die Krümmung des queren karpalen Bogens der Handwurzel kann im Gegensatz zum queren Mittelhandbogen nicht aktiv ausgeglichen werden (Kapandji 1980). 


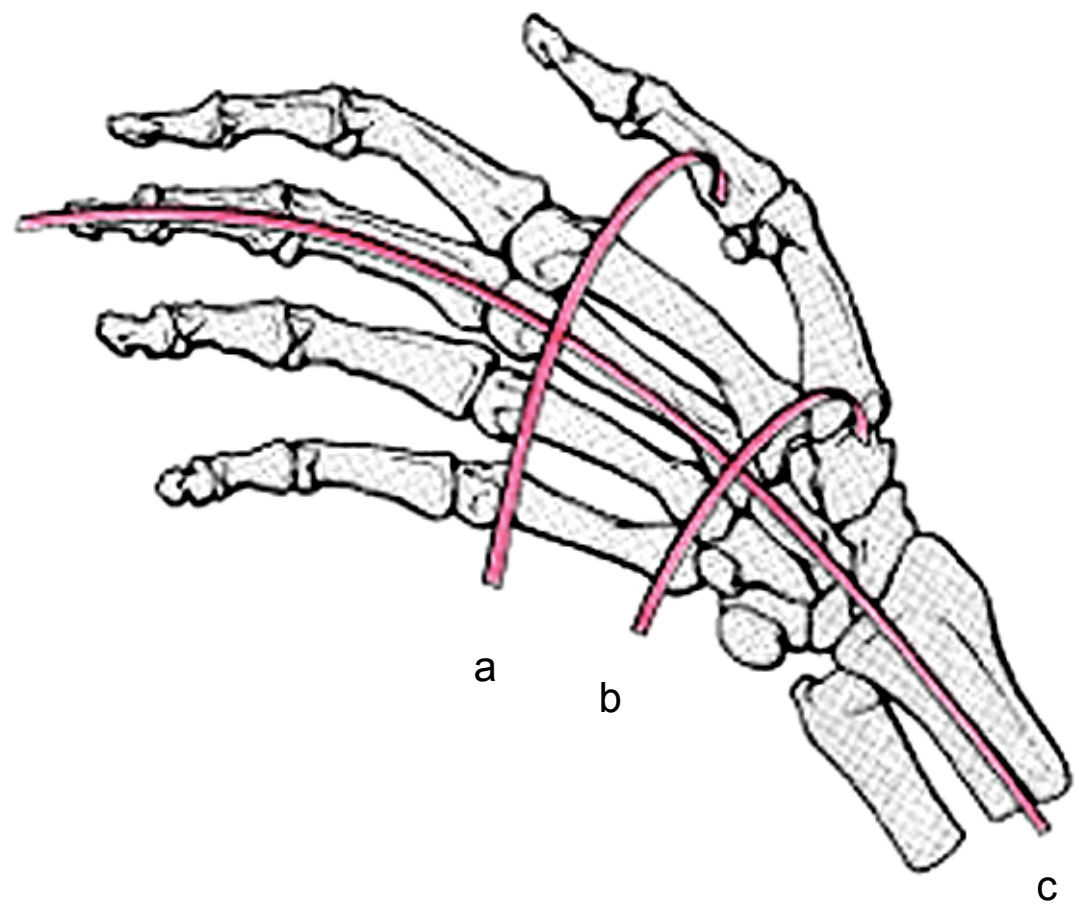

Abbildung 4: Drei Bögen des Handskeletts: a) metakarpaler Bogen, b) karpaler Bogen, c) Längsbogen (Kapandji 1980)

Die Basen der Mittelhandknochen 2 bis 5 sind mit der distalen Handwurzelreihe durch straffe Bänder verbunden (Kapandji 1980). Die Beweglichkeit der Carpometacarpal (CMC) - Gelenke 2 und 3 beträgt weniger als $5^{\circ}$, stabilisiert die Strahlen 2 und 3 aber hierdurch. Die Beweglichkeit des 4. und 5. CMC - Gelenkes beträgt $20^{\circ}$ bis $30^{\circ}$ (Aleksandrowicz et al. 1974, Dubousset 1981, Koebke und Stümpel 1981). Die Kapsel der MCP - Gelenke 2 - 5 ist schlaff, diese Gelenke werden durch kräftige Seitenbänder stabilisiert, die dorsal der Beugeachse liegen. Sie sind in Streckstellung schlaff, in Beugestellung angespannt. Bei einer Immobilisation dieser Gelenke ist deshalb auf eine Beugestellung von ca. $70^{\circ} \mathrm{zu}$ achten, damit es nicht durch Verkürzung dieser Bänder zur Gelenkeinsteifung kommt (Landsmeer 1955). Bei Inspektion der Fingerspitzen in Streckstellung sollten die Fingernägel annähernd parallel stehen. Häufig besteht eine leichte Rotation $\left(3-6^{\circ}\right)$ des Zeige - und Kleinfingers in Richtung Mittelfinger. In Beugestellung der Finger zeigen die Fingerspitzen der Strahlen II und III auf einen gemeinsamen Punkt am Os scaphoideum, die Fingerspitzen der Strahlen IV und V auf einen gemeinsamen Punkt am Os lunatum (Schmidt und Lanz 1992). Bei der Beurteilung von Winkelstellungen und Rotation kann die Hand der Gegenseite als Kontrolle dienen (Schmidt und Lanz 1992). 


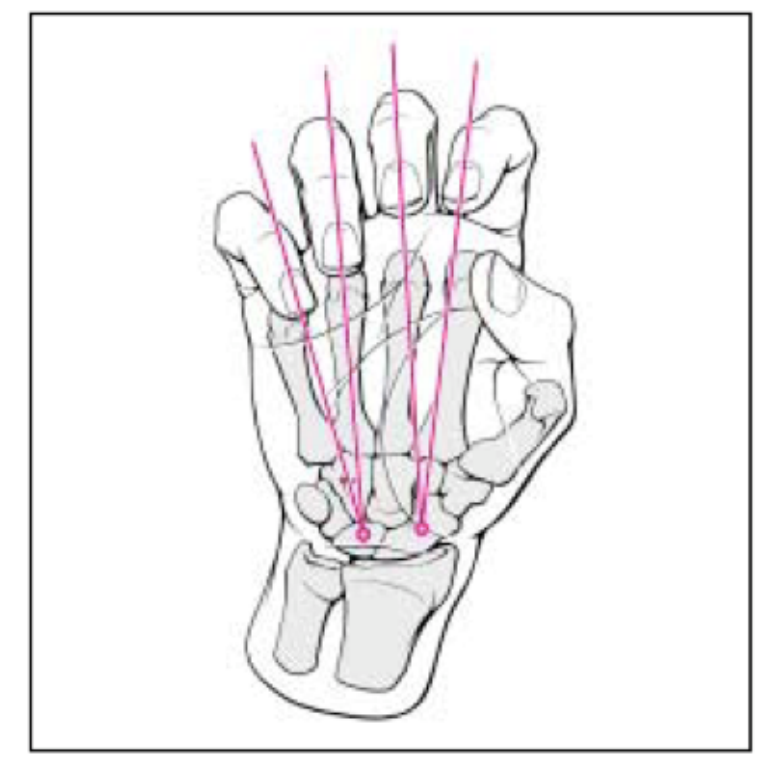

Abbildung 5: Bei Beugung konvergieren die Fingerachsen der Strahlen II und III auf einen gemeinsamen Punkt am Os scaphoideum, die Fingerachsen der Strahlen IV und V auf einen gemeinsamen Punkt am Os lunatum (Schmidt und Lanz 1992).

Mittelhandknochen sind kurze Röhrenknochen, die einen schlanken Schaft aufweisen. Der Schaft ist nach dorsal leicht konvex, nach palmar stärker konkav gekrümmt. An Längs - und Querschnitten durch die Mittelhandknochen erkennt man, daß die Kompaktadicken sehr unterschiedlich angelegt sind (Abbildung 4). Nach Berechnungen von Lazar und Schulter - Ellis 1980 ist die Dicke der Kortikalis aller MHK palmar um 20\% stärker als dorsal (Lazar und Schulter - Ellis 1980). Sie nimmt außerdem nach proximal und distal kontinuierlich ab. Dem entspricht eine mit Spongiosabälkchen durchsetzte weite Markhöhle an den Endabschnitten der MHK. Dagegen ist die Markhöhle im Bereich der Diaphyse des Knochens eng und nicht mit Spongiosa durchsetzt, bedingt durch die Dickenzunahme der Kompakta. Die Diaphysen der MHK II - V sind optimal an Biegebeanspruchungen angepasst. Die Richtung, in der Mittelhandknochen einer Biegebeanspruchung bestmöglich standhalten, verläuft am Zeige-, Mittel- und Ringfinger dorso - radial und am Kleinfinger dorso - ulnar (Lazar und Schulter - Ellis 1980). Die Basis der Mittelhandknochen ist breiter als der Schaft. An ihren radialen und ulnaren Seiten zeigen die Basen weitere stark variierende Gelenkareale, die den Gleitflächen zwischen den Mittelhandknochen entsprechen. Der Basisbereich der Mittelhandknochen ist unregelmäßig gestaltet, da Blutgefässe eintreten und Bänder ansetzen. Den distalen Teil des Mittelhandknochens bildet das Caput metacarpale. Die dorso - palmare Achse der Basis stimmt nicht mit der des Caput überein: Als Folge fällt die Torsion 
der Mittelhandknochen II - V unterschiedlich aus. Die Köpfe des zweiten und dritten MHK sind gegenüber der Basis im Sinne der Pronation, die des vierten und fünften dagegen im Sinne der Supination torquiert (Kapandji 1980, Schmidt und Lanz 1992). Daraus erklärt sich die funktionell wichtige Beobachtung, dass die knöchernen Transversalbögen an der palmaren Seite des Metakarpus proximal stärker, distal schwächer gekrümmt sind (Abbildung 6). Da die Metacarpalknochen von proximal nach distal fächerförmig auseinanderweichen, wird der distale Bogen zusätzlich abgeflacht (Kapandji 1980, Schmidt und Lanz 1992).
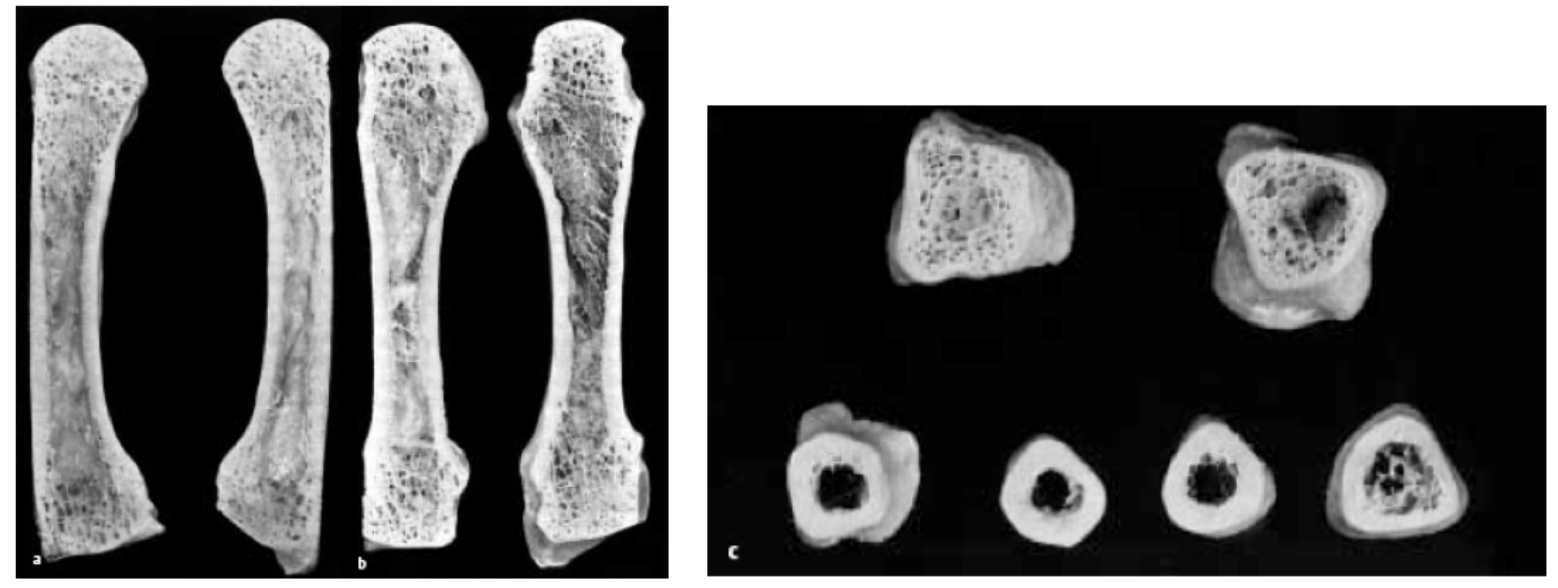

Abbildung 6: Längschnitte in sagittaler Richtung $(a, b)$ und Querschnitte (c) durch humane Mittelhandknochen (Schmidt und Lanz 1992).

\subsubsection{Handbinnenmuskulatur}

\subsubsection{Mm. lumbricales}

In jeder Hand gibt es vier Mm. lumbricales. Die schlanken Muskeln sind in das Bindegewebe zwischen Palmaraponeurose, Beugesehnen und Mittelhandknochen eingebettet. Palmar vom Lig. metacarpale transversum profundum ziehen sie in bindegewebig begrenzten Kanälen zu den Fingern. Die Mm. lumbricales sind aktiv an der Reduzierung der Beugekräfte am Finger und einer Steigerung der Streckwirkung beteiligt (Schmidt und Lanz 1992). Sie werden passiv von den schrägen Landsmeer'schen Retinakula unterstützt (Landsmeer 1955). Die Mm. lumbricales gehören zu den am stärksten variierenden Muskeln im menschlichen Körper. Die Variationshäufigkeit nimmt vom I. bis zum IV. Lumbrikalmuskel sowohl im Ursprung als auch im Ansatz zu. Der M. lumbricalis I löst sich regelhaft von der radialen Seite der Profundussehne für den Zeigefinger. Der Muskel ist einfach gefiedert und zieht zur radialen Seite der Dorsalaponeurose des II. Finger. Er kann auch doppelt ange- 
legt oder zweiköpfig sein, wobei beide Köpfe in der Dorsalaponeurose inserieren. Am Mittelfinger lösen sich ebenfalls von der Radialseite der Profundussehne die Fasern des M. lumbricalis 2, der in 95\% der Fälle einköpfig zur zugehörigen Dorsalaponeurose verläuft. In den übrigen $5 \%$ der Fälle ist der Muskel zweiköpfig. Dies bedeutet einen zusätzlichen Ursprung an der ulnaren Seite der Profundussehne des II. Fingers. Der M. lumbricalis III ist in den meisten Fällen (81\%) doppelt gefiedert. Er entspringt von der radialen Seite der tiefen Beugesehne für den III. Finger. Der Ansatz befindet sich in der Regel radialseitig am Mittelfinger. In der überwiegenden Zahl der Fälle (63\%) entwickelt sich der M. lumbricalis IV zweiköpfig von der tiefen Beugesehne für den IV. Finger (ulnar) und für den V. Finger (radial). Die Insertion des IV. Lumbrikalmuskels liegt zu $85 \%$ an der radialen Seite des Kleinfingers (Frohse und Fränkel 1908).

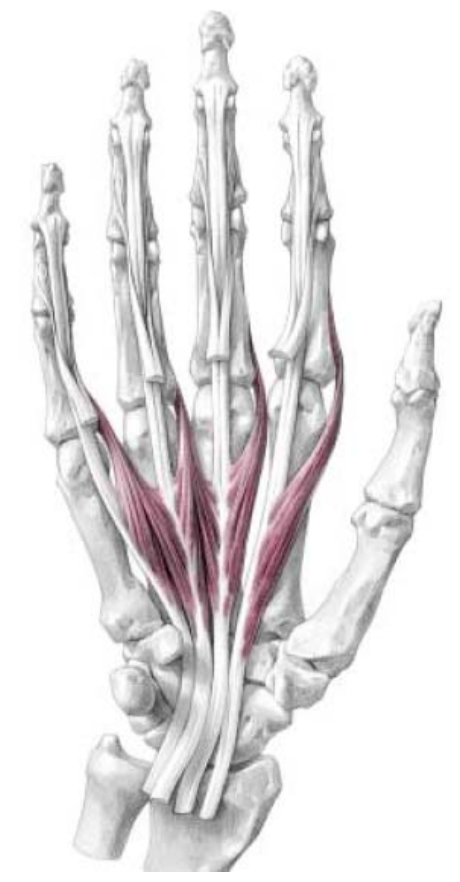

Abbildung 7: Mm. lumbricales (palmar) (Schmidt und Lanz 1992) 


\subsubsection{Mm. interossei}

Entwicklungsgeschichtlich besaß jeder Finger ein Paar kurzer tiefer Beugemuskeln (Frohse und Fränkel 1908). Diese inserierten an beiden Seiten der Grundphalangen oder an den zugehörigen Sesambeinen. Vier von diesen zehn Muskeln verlagerten sich nach dorsal in die interossären Räume zwischen den Mittelhandknochen, Mm. Interossei dorsales. Drei weitere verblieben palmar, Mm. interossei palmares. Radial wurden zwei der kurzen tiefen Beuger in das Caput profundum des M. flexor pollicis brevis bzw. das Caput obliquum des Mm. adductor pollicis aufgenommen. Am Kleinfinger ist der ulnare Muskel im M. opponens digiti minimi enthalten. Die vier Mm. interossei dorsales entspringen zweiköpfig von den jeweils zugewandten Seiten des I. bis V. MHK (Frohse und Fränkel 1908). Der erste dorsale Interosseusmuskel liegt zwischen den MHK des Daumens und des Zeigefingers. Er entspringt als kräftiger radialer Kopf von der ulnaren Kante des Corpus metacarpale I. Der ulnare Kopf entspringt der Basis des II. MHK und der radialen Fläche des Schaftes.

Die Ansatzsehnen vom radialen sowie dem oberflächlichen Teil des ulnaren Kopfes des M. interosseus I ziehen gemeinsam zu ihrem knöchernen Ansatz an der radialen Seite der Basis phalangis proximalis I. Vom ulnaren Kopf löst sich ein tiefer Anteil, der die genannte Ansatzsehne von palmar spiralig eingedreht unterfängt, bevor er zusammen mit dem M. lumbricalis I in die Lamina intertendinea (interosseus hood) der Streckaponeurose einstrahlt. Die übrigen dorsalen Interosseusmuskeln schließen die metacarpalen Zwischenräume bis auf kleine Lücken, die den Rami perforantes der Aa. metacarpales dorsales als Durchtrittpforten zum tiefen Hohlhandbogen dienen. Die drei palmaren Interosseusmuskeln lösen sich einköpfig von den MHK II, IV und V von den jeweils dem Mittelfinger zugewandten Seiten. Sie sind doppelt gefiedert und setzen mit Ihren Ansatzsehnen an der Dorsalaopneurose des Fingers an (Frohse und Fränkel 1908, Schmidt und Lanz 1992). 

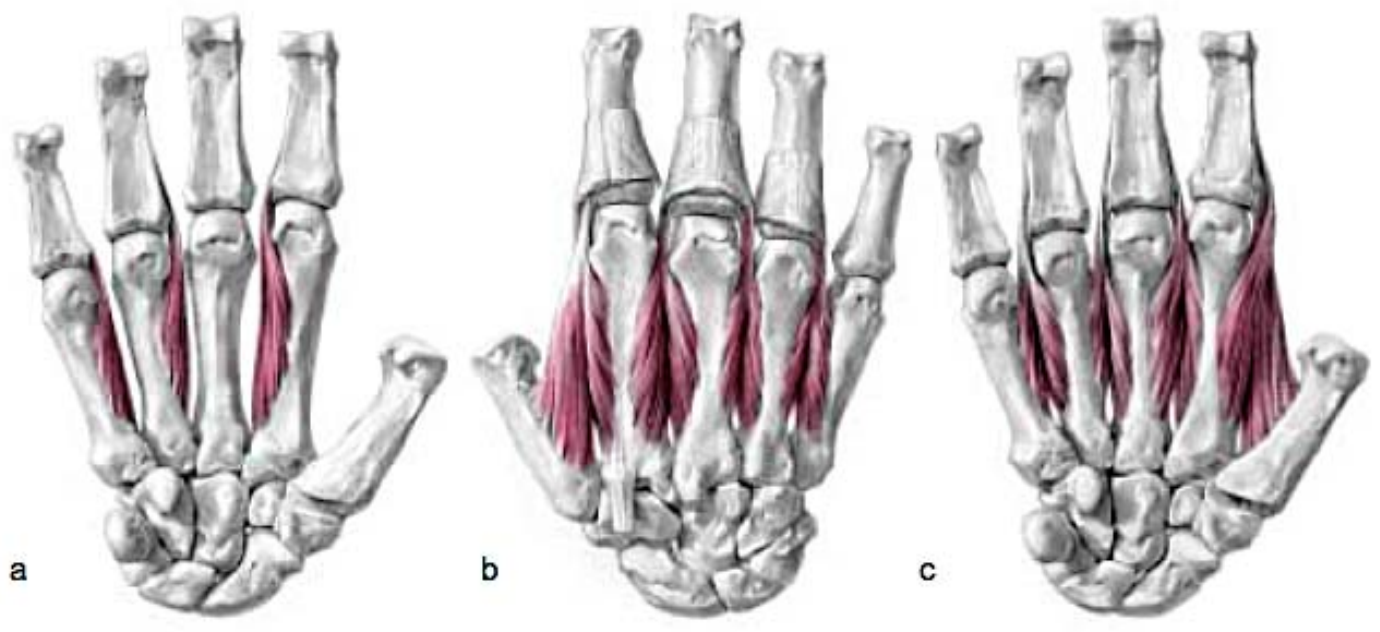

Abbildung 8: $\mathrm{Mm}$. interossei palmares und dorsales: a) $3 \mathrm{Mm}$. interossei palmares (Ansicht von palmar rechts), b) Mm. interossei dorsales superficiales (Ansicht von dorsal rechts), c) Mm. interossei dorsales profundes (Ansicht von palmar rechts) (Schmidt und Lanz 1992).

\subsection{OP-Technik (Plattenosteosynthese, resorbierbares Implantat)}

Die offene Repostion und interne Fixation einer Mittelhandfraktur kann in allgemeiner Intubationsnarkose oder Plexusanästhesie erfolgen. Der Patient wird auf dem Rücken liegend gelagert, die verletzte Hand wird auf einem Armtisch abgelegt, eine Oberarmblutsperre oder Blutleere wird angelegt. Nach dreimaliger Hautdesinfektion und steriler Abdeckung erfolgt die dorsale Längsinzision seitlich des Mittelhandknochens bzw. zwischen den Mittelhandknochen. Extensorsehnen werden beiseite geschoben, peritendinöses Gewebe verbleibt an der Sehne. Anschließend wird die Fraktur durch eine kurze Inzision des Periosts dargestellt und eingeschlagenes Weichteilgewebe entfernt. Die Fraktur wird reponiert. Nach Anlage der resorbierbaren Platte von lateral oder auch dorsal folgt die Rücknaht des Periosts und der Mm. interossei. Eine subtile Blutstillung nach Öffnen der Blutsperre oder Blutleere vor Verschluss der Haut und eine spannungsfreie Hautnaht mit dünnem atraumatischem Nahtmaterial beenden die Operation (Dumont et al. 2007, Nunley und Joneschild 2006, Pechlaner et al. 1998, Strauch et al. 1998).

\subsection{Spezifische Komplikationen bei resorbierbaren Implantaten}

Von den Komplikationen bei chirurgischen Eingriffen, wie Blutung, Nachblutung, neuro - vaskuläre Schädigungen und symphatische Reflexdystrophie abgesehen, 
birgt der Einsatz von resorbierbaren Implantaten zusätzliche Risiken (Hofmann et al. 1996, Hofmann et al. 1990, Hofmann 1997, Hofmann und Wagner 1993, Hofmann 1992, Trevisan et al. 2004, van der Elst 1998, van der Elst et al. 2000, Waris et al. 2002, Waris et al. 2004).

Weiler et al. beschrieben 1996 im Tierversuch bei Merino Schafen das Auftreten von Osteolysen und Bildung von Spontansinus im Rahmen von Fremdkörperreaktion nach Implantation von Polyglycolid (PGA) - Osteosynthesematerial (Weiler et al. 1996). Zwar konnte bei allen Versuchstieren eine primäre Wundheilung erreicht werden, aber bei 6 von 10 Schafen traten fistel - ähnliche Verbindungen und Osteolysen auf. Diese wurden auf die Ansammlung von Makrophagen am Knochen - Implantat - Interface zurückgeführt, die durch den Abbau von Polyglycolid aktiviert wurden. Teilchen, die beim Abbau von Polyglycolid entstehen, werden zuerst von Makrophagen und segmentkernigen Granulozyten phagozytiert. Dies führt möglicherweise zum Absterben von Makrophagen und konsekutiv zu einer Entzündung. Der Zelltod scheint im Zusammenhang mit der Masse phagozytierter Teilchen zu stehen. Diese Vorstellung führte zu dem Schluss, man könne die Biokompatibilität durch einen langsameren Abbau des resobierbaren Materials erhöhen (Becker et al. 1999, Bergsma et al. 1995, Böstmann et al. 1990, Fini et al. 1995, Fuchs et al. 2003, Fuchs et al. 1998, Hofmann et al. 1996, Hofmann et al. 1990, Hofmann 1997, Hofmann und Wagner 1993, Hofmann 1992, Weiler et al. 1996).

Seit Erscheinen von Berichten über Weichteilreaktionen bei PGA - Implantaten wurden vermehrt resorbierbare Implantate bestehend aus Poly - L - Lactid (PLA) verwendet. Obwohl sich diese Implantate aus PLA durch eine bessere Biokompatibilität auszeichnen, bleiben Weichteilreaktionen nicht aus. Die Inzidenz und Intensität der Weichteilreaktionen haben sich jedoch reduziert (Hanemann et al. 2005, Heidemann 2003, Hofmann et al. 1996, Hofmann et al. 1990, Hofmann 1997, Hofmann und Wagner 1993, Hofmann 1992, Kulkarni et al. 1966, Kujala et al. 2004, Kunz et al. 1995, Leenslag et al. 1987, Litsky 1993.)

In einem Update über „Bioabsorbable Implants in the Treatment of Hand Fractures“ beschreibt auch Hughes, dass biodegradierbare Implantate nicht ohne Nachteil sind. So ist die Rigidität sehr ähnlich der von Titanimplantaten, aber eben nicht gleich. Der biochemische Abbau von resorbierbaren Implantaten aus früheren Generationen führt zu einem rapiden Verlust an initialer Stabilität und damit zu einer höheren Inzidenz von Implantatversagen und Refrakturen. Zusätzlich führen bio- 
chemische Prozesse zu synovialen Reaktionen, sterilen Flüssigkeitsansammlungen, Sinusbildung und fibrösen Einkapselungen (Hughes 2006).

Der Entstehungsmechanismus lokaler Fremdkörperreaktionen auf biodegradierbare Implantate ist weiter ungeklärt. Die Theorien über die Auslöser dieser Reaktionen reichen vom beigefügten Farbstoff, über erhöhten osmotischen Druck durch Abbau der Implantate und somit Extrusion von Débris in die Weichteile, $\mathrm{pH}$ Verschiebungen am Implantatlager, bis hin zu lokalen aseptischen Entzündungsreaktionen (Bergsma et al. 1995, Böstmann et al. 1990, Brüser et al. 1999, Mollaoglu 2003, Schmidt und Lanz 1992).

Hoffmann et al. 1997 entwarfen eine Klassifikation zur Einteilung der Fremdkörperreaktionen im extraartikulären und intraartikulären Bereich, sowie dem Auftreten von Osteolysen. Unter Zuhilfenahme dieser Klassifikation soll es möglich sein, die Fremdkörperreaktionen nach möglichst standardisierten Kriterien zu erfassen (Hoffmann 1997).

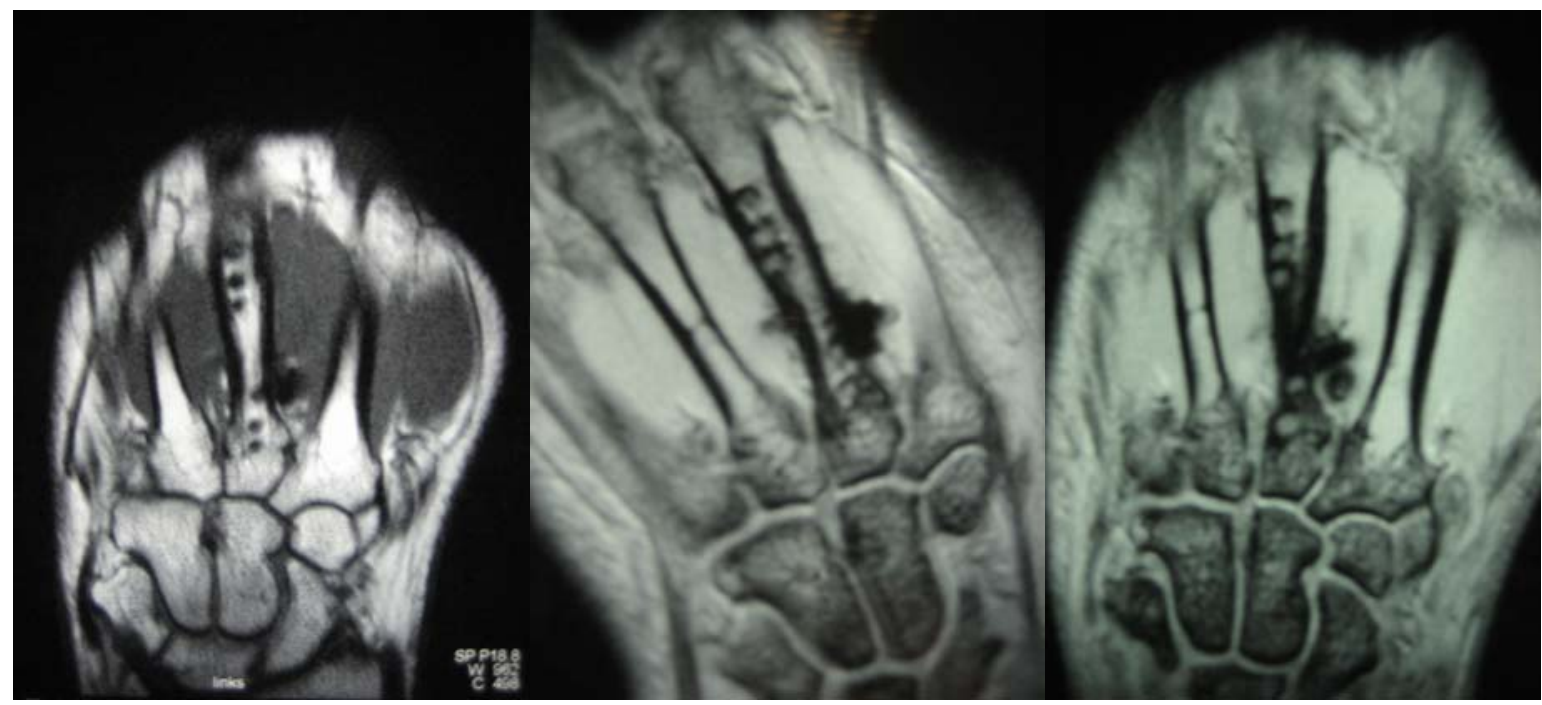

Abbildung 1: Fallbeispiel 2: Magnetresonanztomographie der rechten Hand nach Plattenosteosynthese an MHK III. 12 Wochen postoperativ beklagte der Patient eine neu aufgetretene Schwellung der Hand verbunden mit einem Streckdefizit der Finger. Kernspintomographisch zeigt sich ein hyperintenses Areal im medialen proximalen Schaftbereich von MHK III, dass möglicherweise ursächlich für das Streckdefizit der Finger sein kann. Das Areal könnte bildmorphologisch als Fremdkörperreaktion interpretiert werden.

\subsection{Fragestellung}

Die dorsale Plattenanlage steht mit Komplikationen im Bereich der Strecksenen im Zusammenhang. Die im Rahmen des Abbauprozesses enstehenden Partikel können durch den bradytrophen Extensorsehnenapparat der Mittelhand nicht gut 
aufgenommen werden. Dies kann die Entstehung von Spontansinus und Wundheilungsstörungen begünstigen (Bozic et al. 2001).

Das Osteosyntheseverfahren wurde modifiziert, und die Platte von lateral an den Mittelhandknochen fixiert. Dabei kam die Platte unter der Handbinnenmuskulatur zu liegen, die eine bessere Durchblutung als der Extensorsehnenapparat aufweist (Dumont et al. 2007). Die laterale Plattenlage stellt nicht die stabilste Osteosynthese am Mittelhandknochen dar (Fini et al. 1995, Fuchs et al. 1998, Low et al. 1995, Massengill et al.1979, Massengill et al. 1982, van der Elst et al. 1998, van der Elst et al. 2000).

Somit ergeben sich die folgenden Fragestellungen:

1. Ist das modifizierte Osteosyntheseverfahren ausreichend stabil für die operative Behandlung von Mittelhandfrakturen?

2. Treten unter dem modifizierten Osteosyntheseverfahren Komplikationen, wie Wundheilungsstörungen oder Spontansinusbildungen auf?

\section{Methode}

\subsection{Material und Methoden - klinische Untersuchungen}

\subsubsection{Fallstudien}

\subsubsection{Einschlusskriterien}

In die Studie wurden 16 Patienten bei Mittelhandfraktur II - V im Alter von 18 bis 48 Jahren eingeschlossen (4 Patienten weiblich, 12 Patienten männlich). Insgesamt lagen 18 Mittelhandfrakturen vor. Die Patienten wurden im Zeitraum von 2004 bis 2006 in der Abteilung für Unfallchirurgie, Plastische und Wiederherstellungschirurgie des Universitätsklinikums Göttingen operiert.

\subsubsection{Ausschlusskriterien}

Aus der Studie wurden alle Patienten ausgeschlossen, die nicht in die Studie eingewilligt haben, sowie Patienten unter 18 Jahren. Patienten mit einer Fraktur des I. MHK wurden ebenfalls ausgeschlossen. 


\subsubsection{Fraktur - Klassifikation}

Die Klassifikation der Mittelhandfrakturen erfolgte nach dem Schema der Arbeitsgemeinschaft Osteosynthese AO. Das hier verwendete Schema wurde 1998 von Petracic und Siebert veröffentlicht (Petracic und Siebert 1998).

Es ermöglicht eine genaue alphanumerische Kodierung der Frakturen durch einen insgesamt 6 - stelligen Code nach folgendem Prinzip:

Die 1. Stelle kennzeichnet die Frakturen der Handwurzel, Mittelhand und der Finger mit der Ziffer 7.

Die 2. Stelle legt die vertikale Knochenreihe (Mittelhand und Finger) eines Fingers fest. (Auf die Kodierung der Handwurzel wird aufgrund der geringen Relevanz für diese Arbeit nicht eingegangen.) So wird mit der Nummer 71 die Knochenreihe des I. Strahls (Daumen und MHK I), mit 73 die Knochenreihe des III. Strahls (Mittelfinger und MHK III) gekennzeichnet.

Die 3. Stelle bezeichnet das betroffene knöcherne Segment innerhalb der vertikalen Knochenreihe. Im Gegensatz zur AO - Klassifikation wird hier das Wort Segment nicht für einen Abschnitt innerhalb eines Knochens verwendet, sondern bezeichnet einen einzelnen Knochen. So ist zum Beispiel mit der Zahlenreihe 720 der Mittelhandknochen des II. Strahls, mit 721 die Grundphalanx, mit 722 die Mittelphalanx des Zeigefingers kodiert (Abbildung 10).

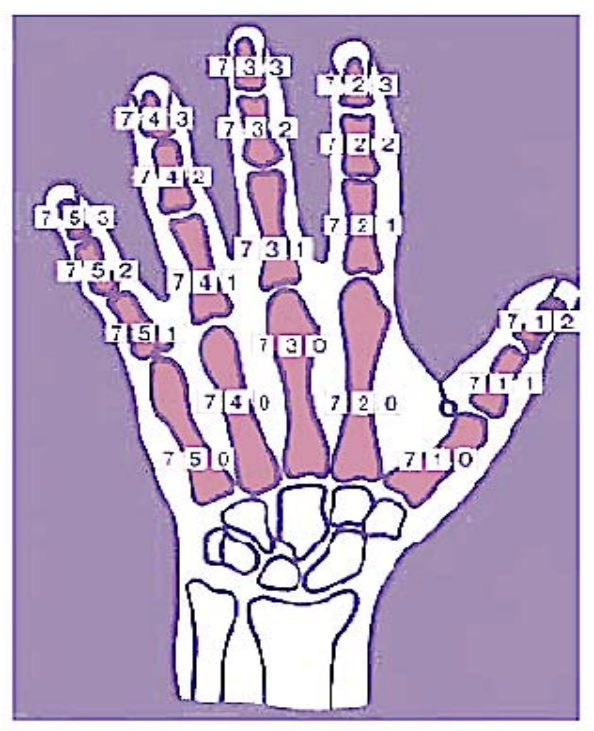

Nummerierung der Knochen der Hand

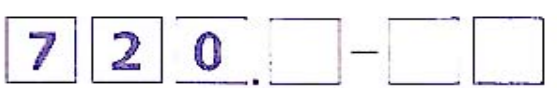

Abbildung 2: Alpha - numerische Kodierung des Handskeletts (Petracic und Siebert 1998) 
Zur genaueren Lokalisation der Fraktur in den einzelnen Knochen wird die vierte Zahl benutzt: 1 = proximal, 2 = diaphysär, 3 = distal.

Mit dem anschließenden 2 - stelligen alphanumerischen Code wird die Lokalisation und Morphologie der Fraktur gekennzeichnet. A entspricht den diaphysären, B den metaphysären und $C$ den Gelenkfrakturen. Gemäß der AO - Klassifikation wird der Frakturtyp in aufsteigender Schwere der Verletzung kodiert. "1" entspricht einer einfachen Zweifragmentfraktur, quer oder spiralförmig schräg, wobei nach Reposition die Fragmente guten Kontakt haben, ohne Defekt. „2" bestimmt eine Fraktur mit einem Biegungskeil oder einem weiteren Fragment. Dieser Frakturtyp weist nach Reposition einen relativ guten Knochenkontakt auf. „3“ entspricht einer Mehrfragment - oder Trümmerfraktur, bei der nach Reposition ein Knochendefekt bleibt.

\subsubsection{Präoperative Vorbereitung}

Nach der schriftlichen und mündlichen Aufklärung sowie dem üblichen präoperativen Procedere (Laborkontrolle, Anästhesieaufklärung), wurden die Patienten durchschnittlich 7,9 Tage (3 - 13 Tage) nach dem Unfall operiert.

\subsubsection{Operative Versorgung}

Die operative Versorgung der Frakturen erfolgte jeweils nach dem „Open Reduction Internal Fixation - ORIF" Verfahren mit resorbierbaren Implantaten LactoSorb® Copolymer 82\% - L - Lactid und 18\% - Glykolsäure der Firma BIOMET durch erfahrene unfallchirurgische Fachärzte oder Handchirurgen der Abteilung für Unfallchirurgie, Plastische und Wiederherstellungschirurgie des Universitätsklinikums Göttingen. Die operativen Eingriffe wurden, wie in Abschnitt 1.11 beschrieben, durchgeführt. Nach durchgeführter Reposition wurde die Fraktur entweder mit resorbierbaren Schrauben oder mit thermoplastischen Platten stabilisiert (LactoSorb® Biomet Deutschland $\mathrm{GmbH}$ ). Diese können nach Erwärmung für ca. 10 Sekunden individuell geformt werden. Gründlicher Blutstillung und Wundspülung folgend wurde eine Saugdrainage der Größe 8 eingelegt und die Wunde schichtweise verschlossen (Dumont et al 2007). 


\subsubsection{Postoperative Weiterbehandlung}

Postoperativ wurde den Patienten protektiv eine Mittelhandorthese (Abbildung 11) verordnet (Küntscher et al 2003, Schleikis 2006).

Nach der stationären Behandlung stellten sich die Patienten in den Abständen 2, 4, 6, 12, 26 und 52 Wochen postoperativ in der Handsprechstunde der Universität Göttingen vor. Die Wundverhältnisse, die Narbenbildung, sowie die Beweglichkeit der einzelnen Fingerstrahlen wurden klinisch untersucht und dokumentiert.

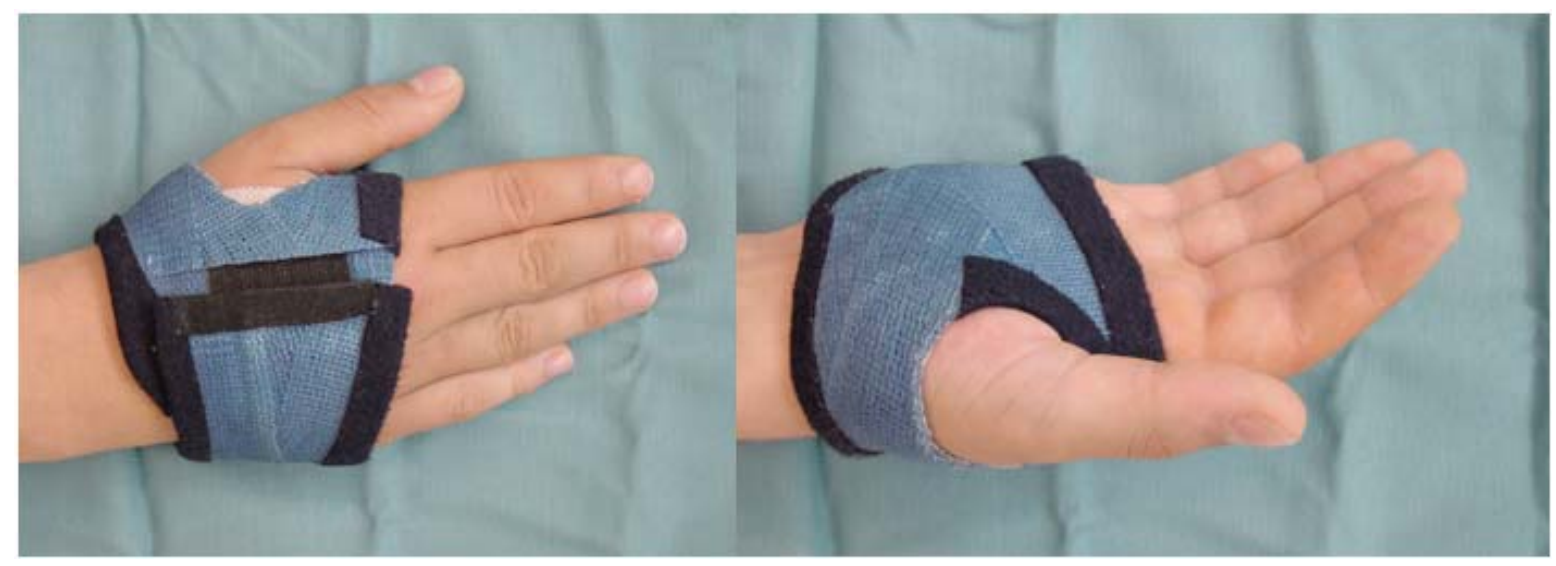

Abbildung 11: Mittelhandorthese bei einer operativ versorgten diaphysären Mittelhandschaftfraktur (Schleikis 2006).

\subsubsection{Postoperative Kontrollen}

In den Abständen 2, 6, 12, 26, 52 Wochen postoperativ wurden gemäß Studienprotokoll Röntgenbilder in zwei Ebenen (posterior - anteriorer und schräger Strahlengang) der betroffenen Mittelhandknochen angefertigt. Die Aufnahmen wurden nach radiologischem Standard, wie unter „1.10 Radiologische Untersuchung bei Frakturen der Mittelhandknochen“ beschrieben, angefertigt.

\subsubsection{Radiologische Auswertungskriterien}

- Knöcherne Konsolidierung der Fraktur

- Abgrenzung des Frakturspalts

- Auftreten von Osteolysen

- Stärke der Kallusbildung 


\subsubsection{Klinische Nachuntersuchung}

Bei den klinischen Untersuchungen wurden folgende Kriterien überprüft (Schmidt und Lanz 1992):

Seitenvergleich: Beim Seitenvergleich soll ein Eindruck über mögliche Symmetrieunterschiede gewonnen werden. Der Patient wurde gebeten, seine Hände in 5 standardisierten Positionen zu halten (Positionen 1 - 5; Abbildung 12):

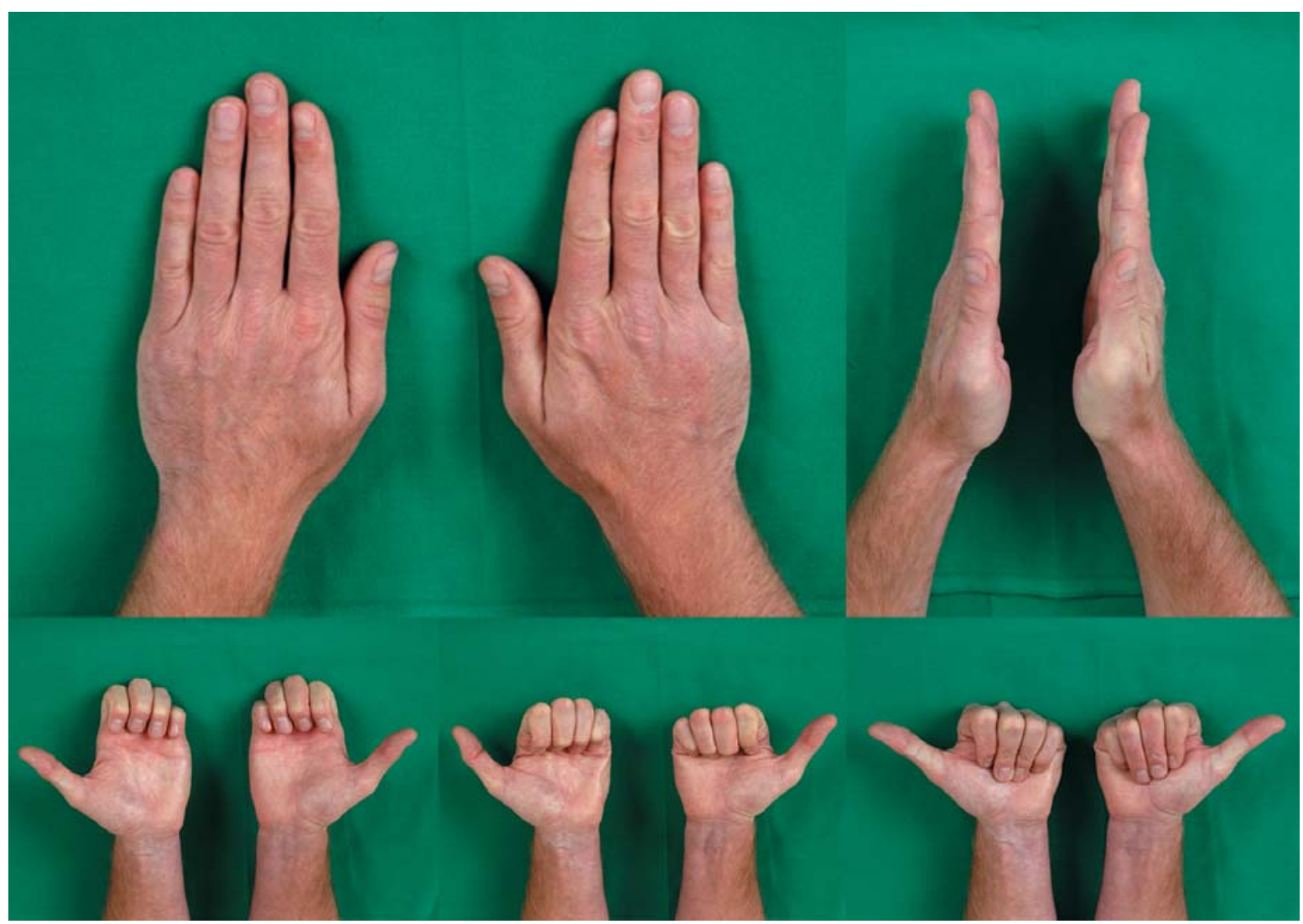

Abbildung 12: Positionen 1-5: Pos. 1: Flachhand im Seitenvergleich; Pos. 2: Handkante zur Beurteilung der Streckung; Pos. 3: Beugung der Finger im proximalen und distalen Interphalangealgelenk (PIP und DIP). Pos. 4: Beugung der Grundgelenke (MCP), PIP und DIP. Die Fingerkuppen berühren die Hohlhandfurche; Pos. 5: Beugung der MCP, PIP ca. $90^{\circ}$. DIP sind gestreckt.

Spitzgriff: Beim Spitzgriff soll die Daumenkuppe die Kuppe der Langfinger berühren.

Fingerkuppenhandabstand: Es wurde der Abstand der Fingerkuppen zur Hohlhandbeugefurche gemessen. Bei gesunden Händen beträgt dieser Abstand $0 \mathrm{~cm}$. Der Fingerkuppenhandabstand wurde für jeden Langfinger der operierten Hand dokumentiert.

Fingernageltischabstand: Der Patient legte seinen Handrücken flach auf den Tisch und versuchte seine Finger möglichst weit zu strecken. Alternativ wurde ein Lineal 
auf den Handrücken gelegt, und der Abstand Fingernagel zu Linealkante gemessen. Hierbei muss die Hand jedoch frei gehalten werden, um eine künstliche Überstreckung der Finger durch das Eigengewicht der Hand bei Auflage auf einen Untergrund zu vermeiden. Auch hier beträgt der Abstand bei gesunden Händen $0 \mathrm{~cm}$. Die Messung des Fingernageltischabstands erfolgte ebenfalls für jeden Langfinger. Fingerabduktion: Die Abduktionsfähigkeit der Langfinger ist abhängig von der Funktion der Handbinnenmuskulatur. Bei der Untersuchung spreizt der Proband die Langfinger gegen den Widerstand des Untersuchers. Es wird der erreichte Kraftgrad dokumentiert.

Fingeradduktion: Die Fähigkeit, die Finger zu adduzieren, wurde in gleicher Weise überprüft und dokumentiert.

\subsection{Visuelle Analogskala (VAS) und DASH - Score}

Die Patienten quantifizierten ihre postoperativen Schmerzen anhand der visuellen Analogskala (VAS) (Scott und Huskisson 1976). Inwieweit sie in der Lage waren, die Routineaktivitäten des alltäglichen Lebens wahrzunehmen, wurde durch den DASH (Disabilities of the Arm Shoulder and Hand) Score erhoben (Germann et al. 1999).

Der DASH - Score ist ein „self - report", der von den Patienten zu Hause oder bei der Untersuchung ausgefüllt werden konnte. Der DASH umfasst die Globalfunktion der oberen Extremität, wobei die beste Funktion mit einem Punkt, die schlechteste mit fünf Punkten bewertet wird. Die Einschätzung der Funktionsminderung errechnet sich aus der Gesamtpunktzahl der Fragen und der Bandbreite des Fragebogens. Einem Punktwert von 100 entspricht die maximale Einschränkung. 0 Punkte entsprechen der freien Funktion (Anhang 6.3), (Germann et al. 1999, Jester et al. 2005).

\subsubsection{Kraftmessung}

Im Rahmen der klinischen und röntgenologischen Untersuchung wurde ab der 12. postoperativen Woche eine Kraftmessung beider Hände mit dem JAMAR® Dynamometer und dem B\&L Engineering PINCH GAUGE® durchgeführt und dokumentiert (Abbildungen 13, 14). Beide Messgeräte haben sich in der Messung der Handkraft bewährt (Bechtol 1954, Mathiowetz et al. 1985). Es liegen Normwerte für die Handkraft bei Erwachsenen vor (Mathiowetz et al. 1985). 
Die Kraftentfaltung der verletzten Hand kann durch multiple Faktoren, wie Schmerzen, Bewegungsablaufstörungen, psychische Faktoren und Fokussierung auf die Verletzung negativ beeinflusst werden. Daraus kann eine übermäßige Schonung wegen mangelnden Vertrauens in die neu erlangte Stabilität nach Frakturheilung resultieren.

Um aus Kraftmessungen aussagekräftige Werte ableiten zu können, müssen diese mehrfach und standardisiert durchgeführt werden. Dies bedeutet, dem Probanden die genaue Handhabung des Untersuchungsgerätes zu erklären und das Untersuchungsgerät, im Falle des JAMAR® Dynamometer, auf die individuelle Handgröße des Probanden einzustellen. Für beide Geräte wurden jeweils 3 Durchläufe in abwechselnder Reihenfolge durchgeführt. Wie in "Hand Strength: Normative Values“ von Crosby et al. beschrieben, wurden nicht die Mittelwerte als Ergebnis verwertet, sondern der bestmöglich erreichte Wert für eine Hand jeweils für das JAMAR® Hand Dynamometer und das B\&L Engineering PINCH GAUGE $®$. Aufgrund der muskulären Ermüdung stellt sich eine Verschlechterung der Messwerte ein (Crosby et al. 1994). Die beste Test - Retest Reliabiliät für dieses Verfahren wurde von Mathiowetz et al. bestätigt (Mathiowetz et al. 1985).

Der Vergleich zur Gegenseite wird erstellt, da ein vorgegebener Messwert naturgemäß nicht existiert. Es muss darauf geachtet werden, dass der Patient zunächst keine Einsicht in die Messergebnisse erhält, um eine willentliche Beeinflussung der Messergebnisse zu vermeiden. Außerdem wurde nach jeder einzelnen Kraftmessung die Seite gewechselt.

Wie von Fess und Moran vorgeschlagen, wurde der Arm in einer standardisierten Position gehalten: Im Sitzen hält der Patient die Schulter adduziert in Neutralstellung, Ellenbogen in $90^{\circ}$ Flexion sowie Unterarm und Handgelenk in Neutralstellung (Fess und Moran 1981, Shyam Kumar et al. 2008).

\subsubsection{JAMAR ${ }^{\circledR}$ Hand Dynamometer}

Zur Messung der Grobkraft wurde das JAMAR® Hand Dynamometer NR verwendet. Es wurde auf die individuelle Handgröße des Patienten eingestellt, so dass die Griffläche zwischen Thenar und den Mittelgliedern der Langfinger lag (Abbildung 13). 


\subsubsection{B\&L Engineering Pinch Gauge ${ }^{\circledR}$}

Mit dem B\&L Engineering Pinch Gauge ${ }^{\circledR}(60 \mathrm{lb})$ ließ sich die Kraftentwicklung zwischen Daumen und einem Langfinger messen. Bei Auflage der Langfingerkuppenspitze und der Daumenkuppenspitze wurde der Spitzgriff überprüft. Mit Auflegen der ulnaren Daumenkuppe und der radialen Zeigefingerkuppe wurde der Schlüsselgriff überprüft (Abbildung 14).

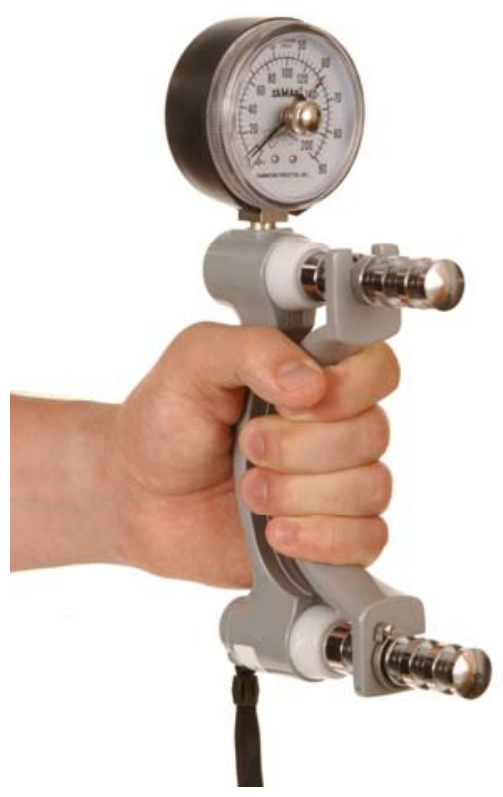

Abbildung 13: JAMAR® Hand Dynamometer

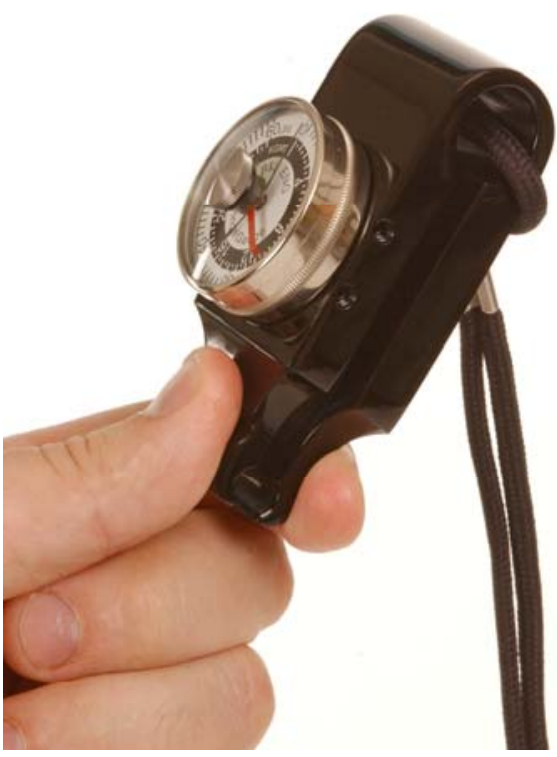

Abbildung 14: B\&L Pinch Gauge $\circledast(60 \mathrm{lb})$ 


\subsection{Material und Methode - biomechanische Versuche}

\subsubsection{Material}

\subsubsection{Tiermodell für humane Mittelhandknochen}

Als Modell für humane Mittelhandknochen wurden frische Mittelfußknochen vom 6 Monate alten Hausschwein verwendet. Der Schweinevorderfuß besteht aus zwei belasteten Mittelstrahlen (III und IV), sowie zwei rudimentären Randstrahlen (II und V), welche ausserhalb der Belastungszone liegen. Form und Beschaffenheit der rudimentären Randstrahlen sind dem humanen Mittelhandknochen ähnlich (Abbildung 15, 16) (Bozic et al. 2001, Massengill et al. 1979, Massengill et al. 1982, van der Elst 2000, Waris et al. 2002, Waris et al. 2004).

Als Modell wurde jedoch nur der II. Mittelfußknochen verwendet, da der V. Mittelfußknochen zumeist verkümmert war. Die Schweinevorderfüße wurden vom Schlachthof Heiligenstadt, Thüringen, bezogen. Im frischen Zustand wurden an 10 Paar Schweinevorderfüßen die II. Mittelfußknochen reseziert, in mit Ringer Lactat - Lösung getränkte Kompressen eingewickelt und einzeln bei $-40^{\circ} \mathrm{C}$ eingefroren.

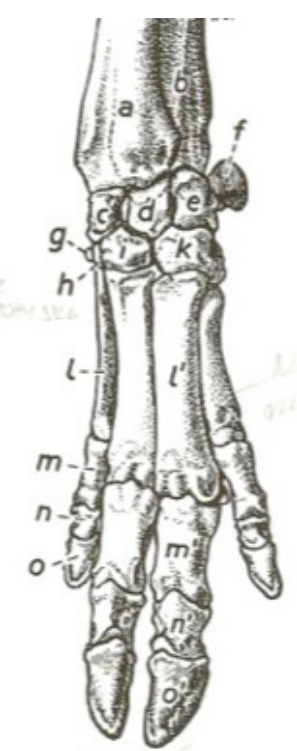

Abbildung 15: anatomische Zeichnung eines Schweinevorderfußes

Die beiden äußeren Strahlen sind rudimentär angelegt und liegen ausserhalb des Belastungsbereichs. 


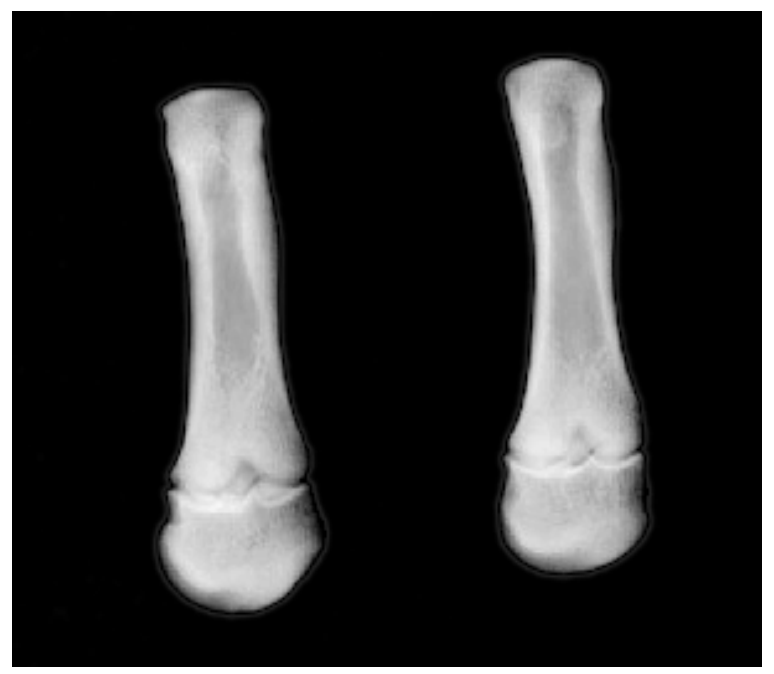

Abbildung 16: Feinschicht Röntgenaufnahme zweier porciner Mittelfußknochen im ap-Strahlengang. Die Epiphysenfugen sind zu erkennen. Die Form der porcinen Mittelfußknochen entspricht der von humanen Mittelhandknochen, somit eignen sie sich gut als Modell (Bozic et al. 2001, Massengill et al. 1979, Massengill et al. 1982).

\subsubsection{Implantate}

Die verwendeten Implantate wurden von Biomet Orthopedics Inc.® (Firma Biomet Merck®) zur Verfügung gestellt. Verwendete Implantate siehe Tabelle 1 und Abbildung 17.

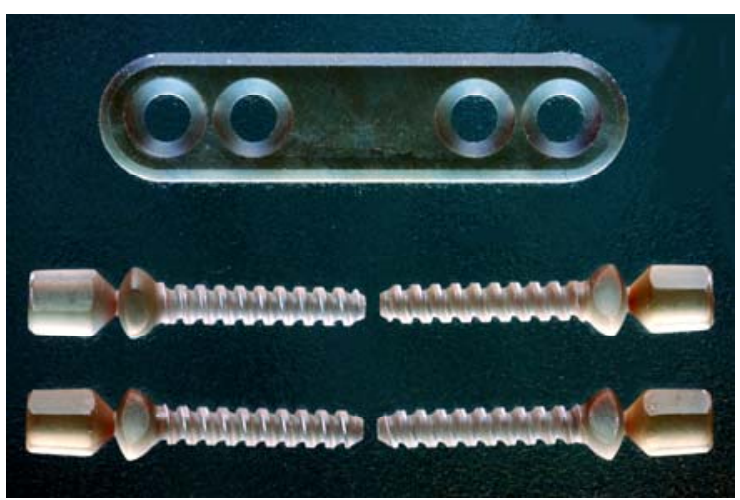

Abbildung 17: Verwendete Implantate

Tabelle 1: Verwendete Implantate

\begin{tabular}{|l|c|}
\hline \multicolumn{1}{|c|}{ Implantat } & Anzahl \\
\hline REUNITE Orthopaedic Plate 4 Hole Straight & 20 \\
REUNITE Orthopaedic Screw $2.0 \times 13 \mathrm{~mm}$ & 12 \\
REUNITE Orthopaedic Screw $2.0 \times 15 \mathrm{~mm}$ & 44 \\
REUNITE Orthopaedic Screw $2.0 \times 17 \mathrm{~mm}$ & 24 \\
\hline
\end{tabular}




\subsubsection{Elastizitätsmodul und Steifigkeit}

Der Elastizitätsmodul (E - Modul) ist ein Materialkennwert aus der Werkstofftechnik, der den Zusammenhang zwischen Spannung und Dehnung bei der Verformung eines festen Körpers bei linear elastischem Verhalten beschreibt (Rösler et al. 2008). Der Elastizitätsmodul hat die Einheit einer Spannung (Elastizitätsmodul [N/mm²]). Anschaulich formuliert, ist der Elastizitätsmodul eines Materials diejenige Zugspannung, bei welcher sich ein Zugstab aus diesem Material in der Länge verdoppelt (Peter Brehm GmbH, Roos und Maile 2008). Der Betrag des Elastizitätsmoduls ist umso größer, je mehr Widerstand ein Material seiner Verformung entgegensetzt (Peter Brehm GmbH). Ein Bauteil aus einem Material mit hohem Elastizitätsmodul $E \approx 2141 \mathrm{~N} / \mathrm{mm}^{2}$ (z. B. Stahl) ist also steif, ein Bauteil aus einem Material mit niedrigem Elastizitätsmodul $E \approx 10 \mathrm{~N} / \mathrm{mm}^{2}$ (z. B. Gummi) ist nachgiebig (Peter Brehm GmbH, Roos und Maile 2008).

Der Elastizitätsmodul im Spannungs - Dehnungs -Diagramm ist als Steigung des Graphen bei einachsiger Belastung innerhalb des linearen Elastizitätsbereichs definiert. Dieser lineare Bereich wird auch als Hookesche Gerade bezeichnet. Die Dehnung ist das Verhältnis von Längenänderung zur ursprünglichen Länge. Der Elastizitätsmodul wird als Materialkonstante bezeichnet, da mit inm und den Querkontraktionszahlen das Elastizitätsgesetz aufgestellt wird. Er hängt von verschiedenen Umgebungsbedingungen, wie Temperatur, Feuchtigkeit oder der Verformungsgeschwindigkeit, ab (Peter Brehm GmbH, Rösler et al. 2008, Roos und Maile 2008).

Dennoch: Für komplexe Geometrien lässt sich kein einfacher Ausdruck für die "Steifigkeit" formulieren (Peter Brehm GmbH). 


\subsubsection{Zwick - Prüfmaschine und Einstellungen}

Die Prüfungen wurden mit einer Materialprüfmaschine der Firma Zwick/Roel|® durchgeführt (Abbildung 18). Die Betriebssoftware testXpert v12.4 wurde verwendet. Es wurden jeweils standardisiert 3 Zyklen am aufgetauten Schweine Mittelfußknochen durchgeführt, im Anschluss wurden je 10 Knochen mit lateral bzw. dorsal angelegter Platte getestet. Die Prüfgeschwindigkeit betrug $0,1 \mathrm{~mm} / \mathrm{s}$. Das Prüfende wurde für das Erreichen der Maximalkraft $F_{\max }\left(F_{\max }=800 N\right)$ oder Bruch des Prüfobjekts definiert.

\subsubsection{Versuchsaufbau in der Prüfmaschine}

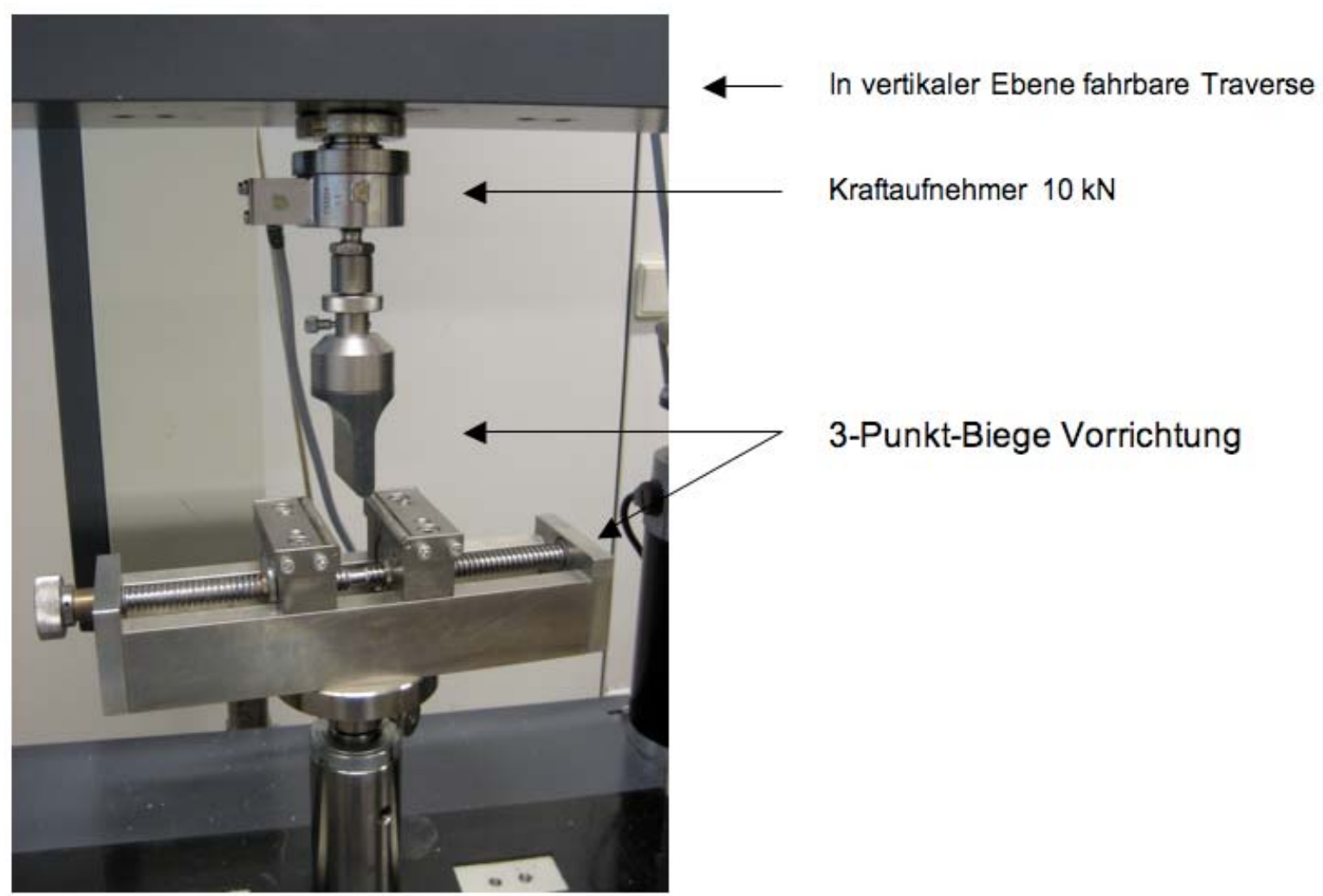

Abbildung 38: 3 - Punkt - Biegeversuchsaufbau in der Zwick® - Prüfmaschine 


\subsubsection{Versuchsdurchführung}

Nachdem die separat eingefrorenen Knochen über 24 Stunden bei $7^{\circ} \mathrm{C}$ aufgetaut waren, wurde bei Zimmertemperatur manuell eine quere Schaftosteotomie durchgeführt (Bozic et al. 2001). Hierzu wurde eine handelsübliche Blattsäge mit Sägelehre verwendet (Abbildung 19).

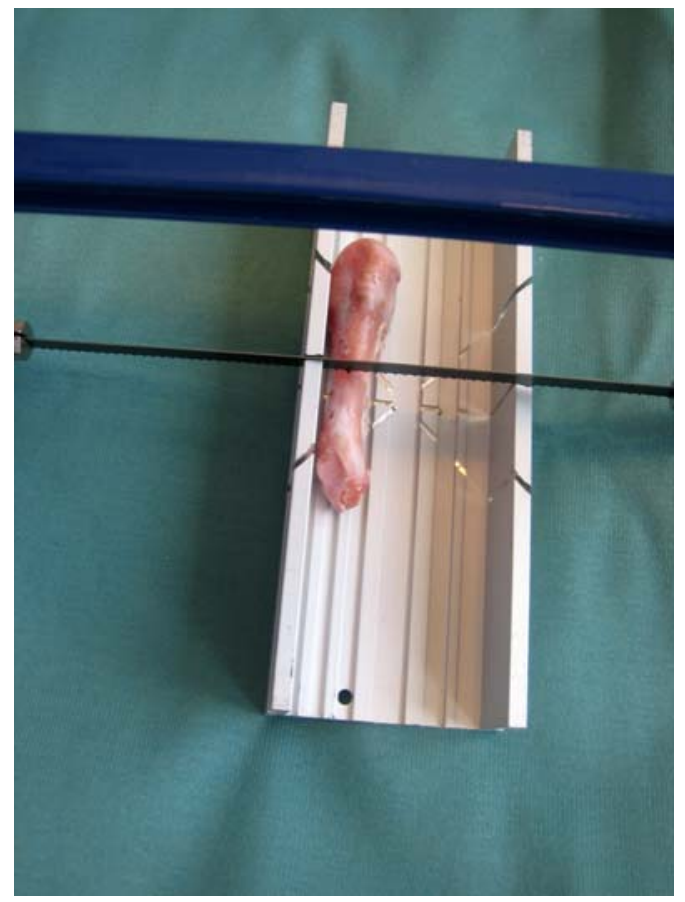

Abbildung 19: Anlegen der queren Mittelschaftosteotomie

Unter Verwendung des ReUnite ${ }^{\mathrm{TM}}$ Orthopaedic Plating System, General Instrumentation V2 (450078) (Abbildung 20) wurden 4 Löcher in die Schweineknochen gebohrt und das Gewinde geschnitten. 


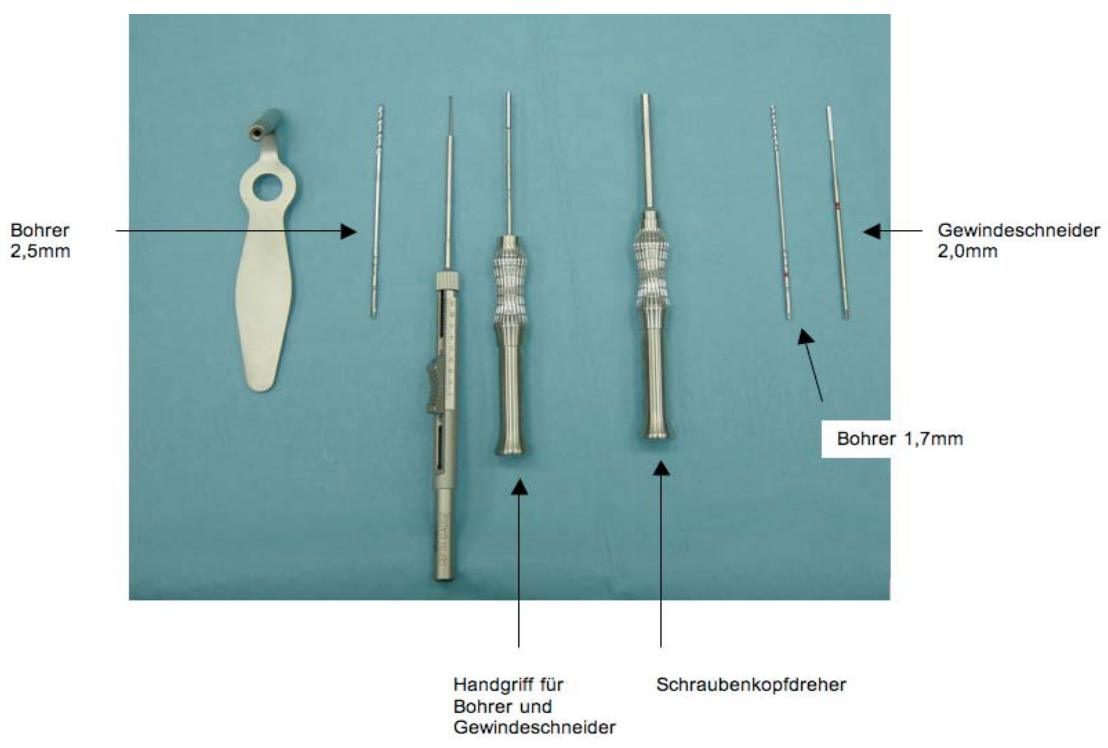

Abbildung 20: Instrumentarium

Anschließend wurde die resorbierbare 4 -Loch Platte mit vier resorbierbaren Schrauben 2,0 mm x 13 - 17 mm am Knochen verschraubt (Abbildung 21).

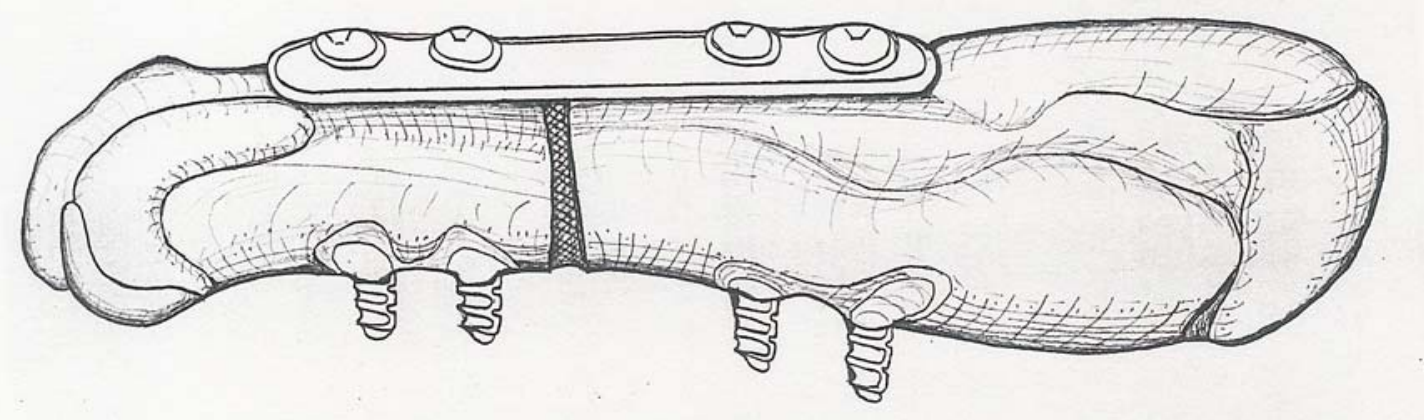

Abbildung 21: Dorso - laterale Plattenanlage nach querer Schaftosteotomie

Nun erfolgte die Einlage des Knochens in die Zwick® - Prüfmaschine, so dass der Stempel mit dem Kraftaufnehmer über der Osteotomie stand. Der Stempel wurde bis auf den Knochen herabgefahren, bis eine Vorspannung von $10 \mathrm{~N}$ anlag. Anschließend wurde die Prüfung mit einer Prüfgeschwindigkeit von 0,1 mm/s gestartet (Bozic et al. 2001).

\subsection{Statistik}

Die erhobenen Daten der klinischen Untersuchung wurden mit dem Statistikprogramm SPSS (Version 15.0, SPSS, USA) deskriptiv, statistisch und graphisch ausgewertet.

Wegen der geringen Stichprobenumfänge waren parameterfreie Tests zur statistischen Auswertung erforderlich. Zur Prüfung, ob die erhobenen Werte einer ge- 
meinsamen Grundgesamtheit angehören, kam der Test nach Kruskal - Wallis für k - unabhängige Stichproben zur Anwendung. Ergaben sich hierfür bestimmte Variablen Signifikanzen, wurden diese mittels Mann - Whitney Test gezielt auf Differenzen untersucht (Signifikanzniveau: $p<0,05)$.

Zur Identifikation von Beziehungen zwischen einzelnen Variablen wurde der Korrelationskoeffizient nach Spearman - Rho $(\rho)$ ermittelt (Signifikanzniveau: $p<0,01$ ). 


\section{Ergebnisse}

\subsection{Ergebnisse - klinische Untersuchung}

\subsubsection{DASH - Score}

Die Einschränkung der Patienten im postoperativen Verlauf wurde mit dem DASH Score erfasst (Germann et al. 1999). Die mittleren Score - Werte, deren Standardabweichungen, Reduktion und Signifikanz (p) sind in Tabelle 2 dargestellt.

Tabelle 2: DASH - Score Ergebnisse

\begin{tabular}{|c|c|c|c|c|}
\hline Zeit & Score - Wert & Standardabweichung & Reduktion & $p$ \\
\hline 2 Wochen & 58,2 & 20,20 & - & - \\
\hline 6 Wochen & 32,3 & 19,40 & $43,9 \%$ & 0,007 \\
\hline 12 Wochen & 11,8 & 11,40 & $63,8 \%$ & 0,010 \\
\hline 26 Wochen & 5,6 & 5,86 & $52,5 \%$ & 0,228 \\
\hline 52 Wochen & 2,96 & 2,44 & $47,1 \%$ & 0,519 \\
\hline
\end{tabular}

In Diagramm 2 sind die DASH - Score - Ergebnisse graphisch dargestellt.

Fehler! Keine gültige Verknüpfung.

Diagramm 2: DASH - Score - Ergebnisse

\subsubsection{Visuelle Analog Skala (VAS)}

Die Auswertung der Visuellen Analog Skala ergab einen zügigen Rückgang der Schmerzverhältnisse im postoperativen Verlauf. Die mittleren VAS - Werte, deren Standardabweichungen, Reduktion und Signifikanz $(p)$ sind in Tabelle 3 dargestellt. Tabelle 3: VAS - Ergebnisse

\begin{tabular}{|c|c|c|c|c|}
\hline Zeit & VAS - Wert & Standardabweichung & Reduktion & $p$ \\
\hline 2 Wochen & 48,5 & 23,0 & - & - \\
\hline 6 Wochen & 23,8 & 15,5 & $50,9 \%$ & 0,012 \\
\hline 12 Wochen & 4,2 & 3,9 & $82,3 \%$ & 0,000 \\
\hline 26 Wochen & 1,1 & 2,0 & $73,8 \%$ & 0,093 \\
\hline 52 Wochen & 0,1 & 0,3 & $90,9 \%$ & 0,282 \\
\hline
\end{tabular}


In Diagramm 3 sind die VAS - Werte graphisch dargestellt.

Fehler! Keine gültige Verknüpfung.Diagramm 3: VAS - Werte

\subsubsection{Klinisch - Radiologische Befunde}

Die Auswertung der Röntgenbilder ergab in $50 \%$ der Fälle eine Fraktur des IV. Strahls. Der III. Strahl war zu 33\%, der V. Strahl zu 11\% frakturiert und zu 6\% der II. Strahl. Die Frakturen waren zu $88 \%$ diaphysär, und zu je $6 \%$ im proximalen und distalen Knochenabschnitt lokalisiert. Am häufigsten (61\%) handelte es sich bei den Mittelhandfrakturen um eine einfache diaphysäre Zweifragmentfraktur (A1), die quer oder spiralförmig schräg verlief. Ein knöcherner Defekt war nach Reposition nicht zu beobachten.

Eine diaphysäre Mittelhandfraktur mit zusätzlichem Fragment oder Biegungskeil (A2) trat in $28 \%$ der Fälle auf.

In 11\% der Fälle lag eine diaphysäre Mehrfragment - oder Trümmerfraktur (A3) vor. Eine metaphysäre Fraktur mit Biegungskeil oder drittem Fragment (B2) konnte zu $6 \%$ beobachtet werden.

Gelenkfrakturen (C1, C2, C3) und weitere metaphysäre Frakturen (B1, B3) konnten nicht festgestellt werden.

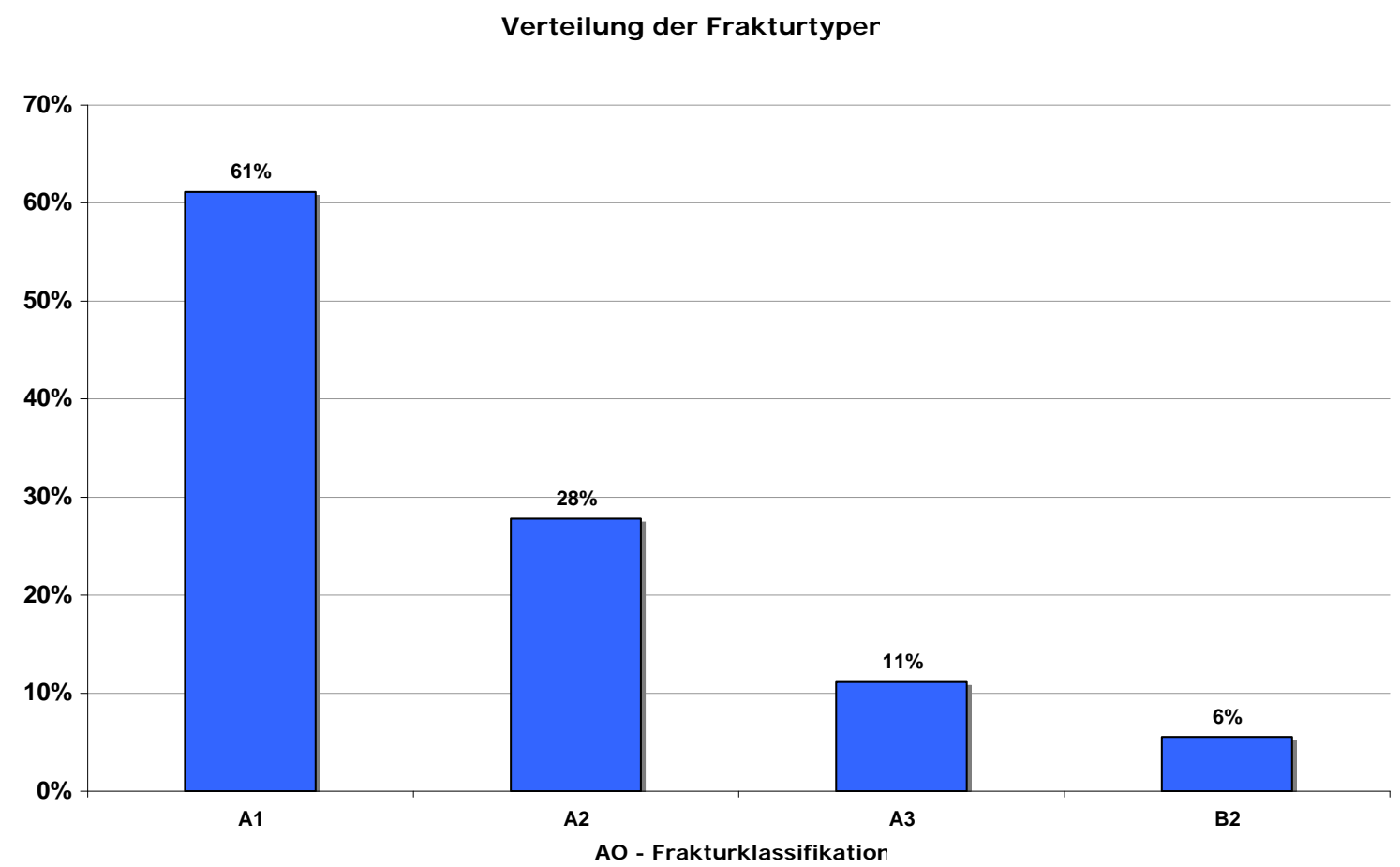

Diagramm 4: Verteilung der Frakturtypen im untersuchten Patientenkollektiv 


\subsubsection{Funktionsprüfung}

Die Untersuchungen der Fingerachsen an der geschlossenen Faust zeigte bei keinem Patienten eine Rotationsabweichung von mehr als $5^{\circ}$. Ein klinisch auffälliger Unterschied zur gesunden Gegenseite war nicht zu beobachten. Die Funktion der Langfinger war uneingeschränkt und seitengleich bei 7 von 8 Patienten nach 12 Wochen.

Nach 12 Wochen postoperativ ließ sich bei einem Patienten ein Flexionsdefizit von $1,5 \mathrm{~cm}$ zur Hohlhandbeugefalte, sowie ein Extensionsdefizit von $4 \mathrm{~cm}$ zur Handrückenebene feststellen. Beide Defizite waren bei klinischer Untersuchung nach 52 Wochen nicht mehr nachweisbar.

Das mittlere Beugedefizit im Patientenkollektiv verbessert sich mit $p=0,421$ bis 0,760 nicht signifikant. Es ist lediglich eine Tendenz erkennbar. Ein erheblicher Rückgang des Beugedefizits ist erst beim Vergleich der Untersuchungsergebnisse nach 2 und 26 Wochen zu verzeichnen $\left(p_{2-26}=0,01\right)$.

Die Rückbildung des Streckdefizits ist nicht signifikant. Die Signifikanzwerte des mittleren Streckdefizits im Patientenkollektiv liegen mit $p=0,401$ bis 0,748 oberhalb des relevanten Niveaus von $p=0,05$. Im Gegensatz zum Beugungsdefizit reduziert sich das Streckdefizit nach 26 Wochen nicht wesentlich.

Fehler! Keine gültige Verknüpfung.

Diagramm 5: Abstand der Fingerkuppe zur Hohlhandbeugefurche (Flexion des frakturierten Strahls)

Fehler! Keine gültige Verknüpfung.Diagramm 6: Abstand der Fingerkuppe zur Handrückenebene (Extension des frakturierten Strahls)

\subsubsection{Kraftmessung}

\subsubsection{JAMAR® Hand Dynamometer}

Die Kraftmessung mit dem JAMAR® Hand Dynamometer an der operierten Hand im Vergleich zur Gegenseite ergab die in Tabelle 4 dargestellten Differenzen ( $\triangle F)$. 
Die signifikante Veränderung $(p)$ trat zwischen der 12. und 26. postoperativen Woche ein. Die übrigen Kraftunterschiede waren nicht signifikant.

Bei einem Patienten war ein Kraftdefizit von $32 \%$ nach 52 Wochen $(30 \mathrm{~kg} \times$ ms und $44 \mathrm{~kg} \times \mathrm{ms}$ ) festzustellen.

Tabelle 4: Kraftunterschied JAMAR® Hand Dynamometer (SD = Standardabweichung)

\begin{tabular}{|c|c|c|c|c|c|c|}
\hline Zeit & $\begin{array}{c}\text { Operierte Hand } \\
(\mathrm{kg} \times \mathrm{ms})\end{array}$ & $\mathrm{SD}$ & $\begin{array}{c}\text { Gesunde Hand } \\
(\mathrm{kg} \times \mathrm{ms})\end{array}$ & $\mathrm{SD}$ & $\triangle \mathrm{F}$ & $\mathrm{p}$ \\
\hline 12 Wochen & 31,8 & 9,5 & 44,3 & 12,8 & $28 \%$ & - \\
\hline 26 Wochen & 36,9 & 11,3 & 42,9 & 12,7 & $14 \%$ & 0,041 \\
\hline 52 Wochen & 40,5 & 13,0 & 43,7 & 13,0 & $7 \%$ & 0,438 \\
\hline
\end{tabular}




\subsubsection{B\&L Engineering PINCH GAUGE®}

In Tabelle 5 sind die Ergebnisse der Kraftmessung mit dem B\&L Engineering PINCH GAUGE $®$ an der operierten Hand im Vergleich zur Gegenseite aufgeführt. Eine signifikante Kraftdifferenz konnte nicht nachgewiesen werden.

Tabelle 5: Kraftunterschied B\&L PINCH GAUGE®

\begin{tabular}{|c|c|c|c|c|c|c|}
\hline Zeit & $\begin{array}{c}\text { Operierte Hand } \\
(\mathrm{kg} \times \mathrm{ms})\end{array}$ & $\mathrm{SD}$ & $\begin{array}{c}\text { Gesunde Hand } \\
(\mathrm{kg} \times \mathrm{ms})\end{array}$ & $\mathrm{SD}$ & $\triangle \mathrm{F}$ & $\mathrm{p}$ \\
\hline 12 Wochen & 9,3 & 3,1 & 11,3 & 3,1 & $17 \%$ & - \\
\hline 26 Wochen & 8,9 & 1,6 & 10,2 & 1,5 & $13 \%$ & 0,424 \\
\hline 52 Wochen & 9,7 & 1,5 & 10,3 & 1,7 & $6 \%$ & 0,076 \\
\hline
\end{tabular}

In Diagramm 7 ist die postoperative Entwicklung der Handkraft gemessen mit dem JAMAR® Hand Dynamometer und dem B\&L Engineering PINCH GAUGE $®$ graphisch dargestellt.

Fehler! Keine gültige Verknüpfung.Diagramm 7: Dargestellt ist die Entwicklung der Kraftzunahme der operierten Mittelhand (Fraktur) über insgesamt 38 Wochen. Es werden annähernd gleiche Werte wie auf der gesunden Seite (GS) erreicht. Dies gilt sowohl für die Messung mit dem JAMAR® Hand Dynamometer, als auch für die Messung mit dem B\&L Engineering PINCH GAUGE®.

\subsubsection{Radiologische Befunde}

\subsubsection{Knöcherne Konsolidierung}

Mittels konventioneller Röntgenbildgebung der Mittelhand in 2 Ebenen wurde die knöcherne Konsolidierung der untersuchten Mittelhandfrakturen nach 6 Wochen bei 11 von 15 Patienten festgestellt. Nach 12 Wochen war die Konsolidierung bei 3 weiteren Patienten eingetreten.

\subsubsection{Kallus und Frakturspaltabgrenzung}

Die Kallusbildung war nach 6 Wochen radiologisch bei 7 von 15 Patienten festzustellen. Nach 12 Wochen war sie bei 11 von 15 Patienten nachweisbar. Der Kallus bildete sich nach 12 Wochen am stärksten aus und erreichte dabei Kortikalisstärke. Der Mittelhandknochen. Danach zeigte sich eine Abnahme des Kallus.

Der Frakturspalt war bei allen Patienten bis einschließlich 6 Wochen abgrenzbar. Nach 12 Wochen ließ sich der Frakturspalt noch bei 10, nach 26 Wochen noch bei 
7 und nach 52 Wochen noch bei 4 Patienten abgrenzen. Danach waren die Frakturspalten nicht mehr erkennbar.

Osteolysen, Pseudarthrosen und Sinusbildungen waren nicht zu beobachten. 


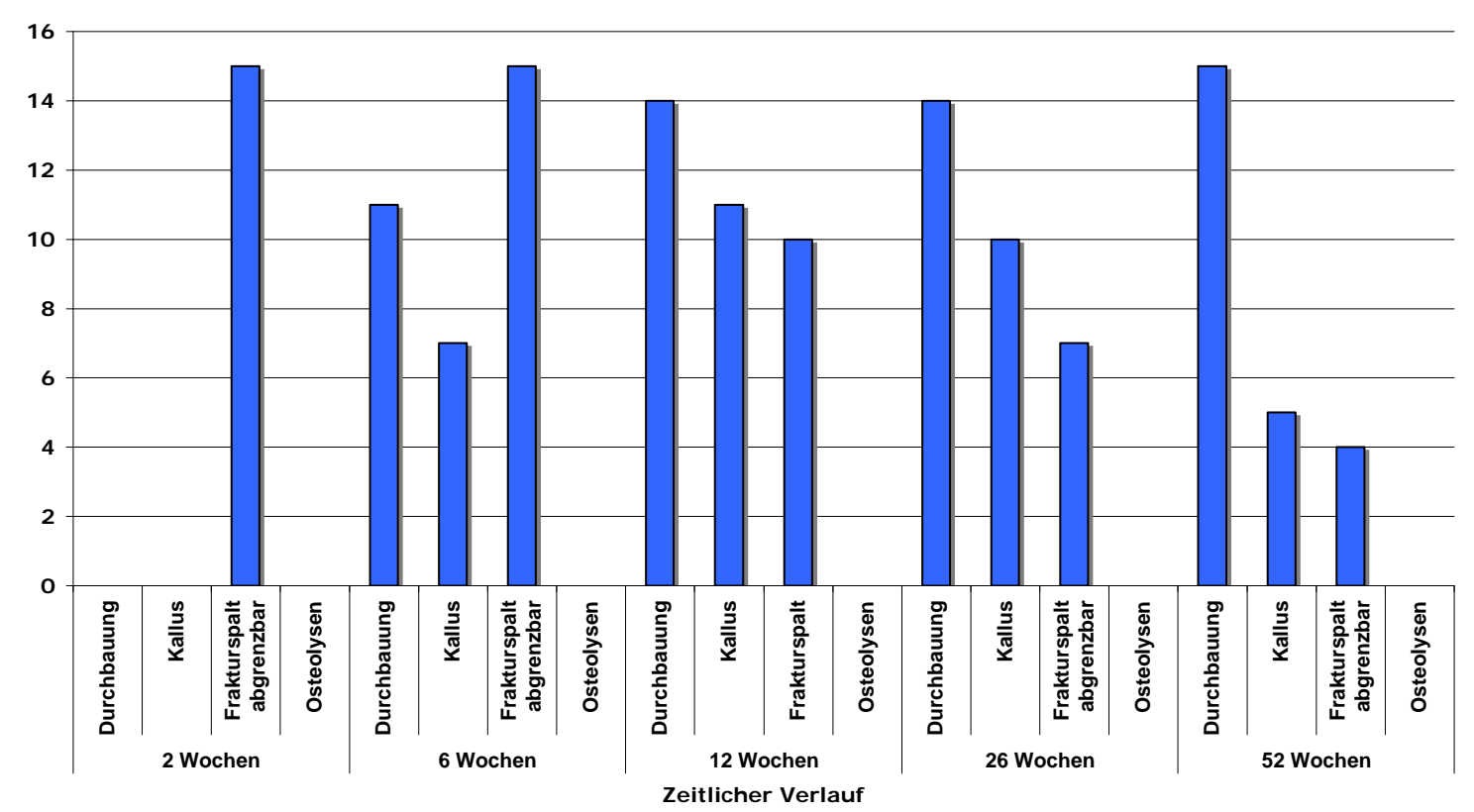

Diagramm 8: Röntgenbefunde

Konsolidierung: Eine knöcherne Durchbauung war ab der 6 . Woche postoperativ zu erkennen (11 von 15 Patienten). Ab der 12. Woche ist dieses bei 14 von 15 Patienten festzustellen.

Kallus: Kallusbildung war ebenfalls ab der 6 . Woche postoperativ zu verzeichnen. Allerdings nur bei einigen Patienten ( 6 Wochen: 7 von 15 Patienten; 12 Wochen: 11 von 15 Patienten; 26 Wochen: 10 von 15 Patienten; 51 Wochen 5 von 15 Patienten).

Frakturspaltabgrenzung: Der Frakturspalt war bis zur 6. Woche postoperativ bei allen Patienten abgrenzbar. Erst danach wurde dies immer seltener möglich (12 Wochen: 10 von 15 Patienten; 26 Wochen: 7 von 15 Patienten; 52 Wochen: 4 von 8 Patienten)

Osteolysen: Es wurden keine Osteolysen beobachtet. 


\subsection{Ergebnisse - biomechanische Untersuchung}

\subsubsection{Elastizitätsmodul}

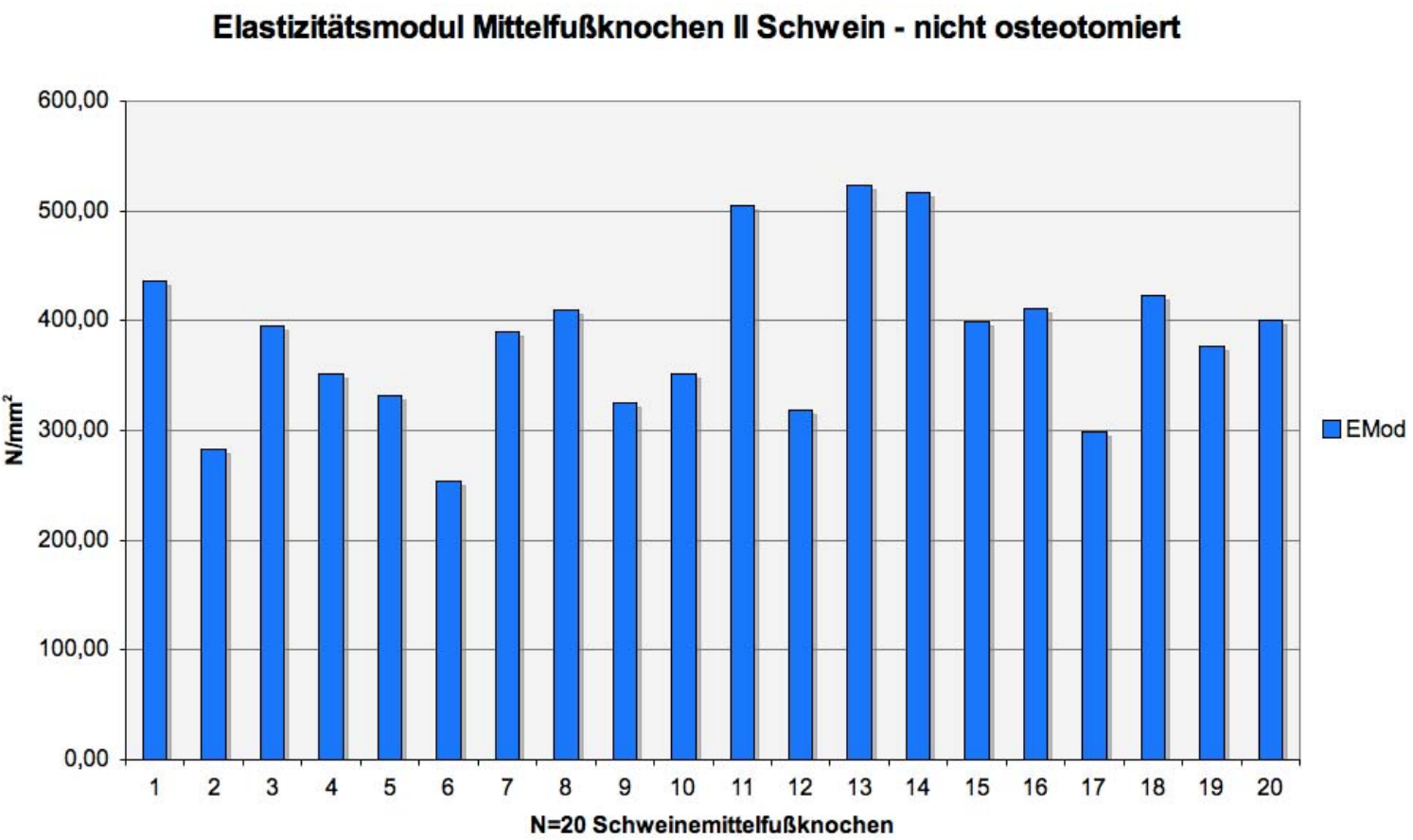

Diagramm 9: Ermittelte Werte des Elastizitätsmoduls

Der maximale Wert beträgt 522,97 N/mm², der minimale $254,35 \mathrm{~N} / \mathrm{mm}^{2}$. Im Mittel liegt der Elastizitätsmodul bei $385,04 \mathrm{~N} / \mathrm{mm}^{2}$ (SD 72,34 N/mm²). 


\subsubsection{Belastungsprüfung bei lateraler Plattenlage}

\section{Fehler! Keine gültige Verknüpfung.Diagramm 10}

Es sind die ermittelten maximalen Kraftwerte dargestellt, die beim Bruch der Platte auftraten. Die maximal erreichte Kraft liegt bei 307,03 N, die minimale bei 22,41 N. Der Mittelwert beträgt 122,05 N (SD 93,12 N). 


\subsubsection{Belastungsprüfung bei dorsaler Plattenlage}

\section{Fehler! Keine gültige Verknüpfung.Diagramm 11}

Es sind die maximal erreichten Kräfte dargestellt, die bei dorsaler Plattenlage beim Bruch des Implantats auftraten. Der maximale Wert beträgt 394,21 N, der minimale 7,89 N. Der Mittelwert liegt bei 133,37 N (SD 113,67 N). 


\section{Diskussion}

\subsection{Einleitung}

\subsubsection{Einschlusskriterien}

Das von uns untersuchte Patientenkollektiv ist mit Patientenkollektiven in der Literatur vergleichbar (Schaefer und Siebert 2000).

\subsubsection{Operative Versorgung}

Zur Vermeidung von Fremdkörperreaktionen wurden die resorbierbaren Platten von dorso - lateral an den frakturierten Mittelhandknochen fixiert, auch wenn dies nicht der maßgeblichen Zugrichtung entspricht (Fini et al. 1995, Fuchs et al. 1998, Fuchs et al. 2003, Litsky 1993, Massengill et al. 1979, Massengill et al. 1982, van der Elst 2000, Waris et al. 2002, Waris et al. 2004). Die Vermeidung von Fremdkörperreaktionen und eine suffiziente Weichteildeckung des Implantats standen im Vordergrund.

\subsection{Methodik}

\subsubsection{Methodik - klinische Untersuchungen}

\subsubsection{Funktionsprüfung}

Die Bewegungsuntersuchung stellt im klinischen Alltag ein wesentliches Instrument zur Beurteilung der Handfunktion dar. Die Untersuchung der Beugung in Bezug auf den Abstand der Fingerkuppe zur Hohlhandfurche und der Streckung in Bezug auf den Abstand der Fingerkuppe zur Handrückenebene lassen sich aufgrund der einfachen und standardisierten Untersuchung gut verwerten. Auf die Untersuchung der Fingerabduktion und - adduktion wurde weitgehend verzichtet (Haerle et al. 2002). Die klinische Untersuchung der Hand basiert zu großen Teilen auf der Erfahrung des Untersuchers, dennoch können sich große Unterschiede in Qualität und Interpretation der Untersuchung und deren Ergebnis ergeben, wenn mehrere Untersucher den gleichen Patienten untersuchen. Um diesen Effekt zu vermeiden, wurden alle Patienten von dem selben Handchirurgen untersucht. 


\subsubsection{Kraftmessungen}

\subsubsection{JAMAR® Hand Dynamometer}

Die MHK spielen als handstabilisierende Elemente eine zentrale Rolle bei der Kraftübertragung. Eine Beeinträchtigung der Handkraft ließ sich somit im Seitenvergleich gut untersuchen (Strauch et al. 1998). Die Beurteilung der Handkraft mit dem JAMAR® Hand Dynamometer ist als objektiv und gut reproduzierbar zu werten (Haerle et al. 2002). Einzige Besonderheit stellt die Einstellung auf die individuelle Handgröße dar. Im klinischen Verlauf wurde außerdem die Einstellung berücksichtigt, in der eine bestmögliche Kraftentfaltung möglich war. Erfreulicherweise hatten 12 von 13 Patienten annähernd gleiche Handgrößen, sodass eine Änderung der Grundeinstellung nur für eine Patientin notwendig war. Die postoperative Kraftentwicklung mit dem JAMAR® Hand Dynamometer lässt sich besonders für Frakturen der Mittelhandknochen gut beobachten (Haerle et al. 2002).

\subsubsection{B\&L Engineering PINCH GAUGE®}

Die Kraftmessung mit dem B\&L Engineering PINCH GAUGE® lieferte sehr ähnliche Ergebnisse wie die Untersuchung mit dem JAMAR® Hand Dynamometer. Die gemessenen Werte lagen durchweg oberhalb des Signifikanzniveaus von $p<0,05$. Die Messung mit dem B\&L Engineering PINCH GAUGE $®$ erlaubt eher eine Beurteilung der speziellen Greiffunktion, wie z.B. dem oben genannten Schlüsselgriff. Eine Untersuchung mit dem B\&L Engineering PINCH GAUGE® erscheint daher wenig spezifisch, ist aber als Ergänzungsuntersuchung einsetzbar (Haerle et al. 2002).

\subsubsection{Methodik - biomechanische Versuche}

Die biomechanische Untersuchung der lateral angebrachten resorbierbaren Platten sollte einen Vergleich zur Plattenosteosynthese von Mittelhandknochen mit Metallimplantaten liefern, die in der maßgeblichen dorsalen Lage fixiert wurden (Freeland und Jabaley 1998, Freeland und Orbay 2006, Freeland et al. 2001, Gautier et al. 2000). In der biomechanischen Studie von Bozic et al. wurde ein 4 -Punkt Versuchsaufbau mit einer Prüfgeschwindigkeit von $0,05 \mathrm{~mm} / \mathrm{s}$ verwendet (Bozic et al. 2001). 
Der hier verwendete 3 - Punkt - Versuchsaufbau hat folgende Nachteile:

- Bei Prüfung der dorsalen Plattenanlage wirkte die Kraft von oben senkrecht auf die Platte ein. Dabei entstand eine nach dorsal wirkende Gegenkraft, die nicht der physiologischen Hauptbelastungsrichtung entspricht. Diese richtet sich nach palmar (Bozic et al. 2001, Gautier et al. 2000).

- Die Epiphysen wurden nicht am Versuchsaufbau fixiert. Sie konnten sich auf der Versuchsplattform frei ausrichten. Dadurch wurde die von oben senkrecht wirkende Kraft nicht vollständig auf den osteosynthetisierten Knochen übertragen.

- Die sehr schmale dorsale Kante der Schweinemittelhandknochen ließ nur eine uneinheitliche Fixierung der dorsalen Platte zu.

\subsection{Ergebnisse - klinische Untersuchung}

\subsubsection{Bewegungsuntersuchung}

Die Bewegungsuntersuchung lieferte einheitliche Ergebnisse, d.h. im Heilungsverlauf waren zum Abschluss der Nachbehandlung bei Streckung in der Handrückenebene keine, und bei Beugung zur Hohlhandebene nur noch sehr diskrete Einschränkungen feststellbar.

\subsubsection{DASH - Score und VAS}

Der DASH - Score und die visuelle Analogskala (VAS) eignen sich gut zur Erfassung der postoperativen Einschränkungen nach standardisierten Kriterien (Germann et al. 1999). Die Zufriedenheit der Patienten kommt dadurch zum Ausdruck und wird gleichzeitig in numerischen Werten ausgedrückt (Germann et al. 1999, Jester et al. 2005).

In der vorgestellten Studie wurde der DASH - Score Fragebogen auf 30 Fragen reduziert, um lediglich die Einschränkung der Hand zu erfassen und Beeinträchtigungen der Schulter auszugrenzen. Auf den speziellen Teil zur besonderen Berücksichtigung von Sportlern und Musikern wurde verzichtet, da sich keine Sportler und Musiker in unserem Kollektiv befanden. Jester et al. beschrieben, dass zwischen den DASH - Score - Werten und der Bewegungseinschränkung (Fingerkuppenhohlhandabstand bei Flexion, Fingerkuppenhandrückenabstand bei Extension) keine signifiante Korrelation bestand. Eine umgekehrt proportionale signifikante 
Korrelation zwischen den DASH - Score - Werten und der Handkraft konnte jedoch nachgewiesen werden (Jester et al. 2005).

Die VAS wurde von den Patienten positiv angenommen. Sie eignet sich hervorragend als Verlaufskontrolle, da die Patienten nicht an numerische Werte gebunden sind (Scott und Huskisson 1976).

Die Reduktion des VAS - Wertes von 48,5 (2 Wochen) auf 23,8 (6 Wochen) stellt den signifikantesten Rückgang der Schmerzen dar $(p=0,013)$. Die weitere Verringerung auf 4,2 (12 Wochen) ist ebenfalls signifikant $(p=0,001)$. Die Patienten schilderten geringe Schmerzen im Bereich zwischen der 6. und 12. postoperativen Woche (32,3 nach 6 Wochen (SD 19,4), 11,8 nach 12 Wochen (SD 11,4), pVAS 6-12Wo $=0,01$. Die Ergebnisse nach 26 bzw. 52 Wochen liegen mit Werten von 5,6 (26 Wochen) und 2,96 (52 Wochen) über dem Signifikanzniveau von $p<0,05$, zeigen aber noch eine Tendenz in Richtung einer leichten Reduktion der Punktwerte.

26 bzw. 52 Wochen postoperativ liegen die Ergebnisse ebenfalls über dem Signifikanzniveau von $p<0,05$. Die Schmerzen verbessern sich nur noch wenig, aber die Schmerzen sind nach 26 und 52 Wochen äußerst gering bzw. die Patienten sind schmerzfrei.

\subsubsection{Kraftmessungen}

Diagramm 7 stellt die Kraftentwicklung der operierten Mittelhand im postoperativen Verlauf im Vergleich zur gesunden Gegenseite dar. Nicht berücksichtigt wurde Links - oder Rechtshändigkeit, Beruf, Alter und Geschlecht der Probanden.

\subsubsection{JAMAR® Hand Dynamometer}

Die 1993 in einer Studie von Crosby et al. (Hand Strength: Normative Values) erarbeiteten Normwerte für Kraftmessungen mit dem JAMARß Hand Dynamometer stellen eine gute Orientierung für die Beurteilung unserer Ergebnisse dar (Crosby et al. 1993). In der Studie von Crosby et al. wurden nur Patienten untersucht, die seit mehr als 5 Jahren keine Handverletzung erlitten hatten. Für einen Vergleich unserer Ergebnisse dürfen also nur die Abschlussergebnisse nach 52 Wochen herangezogen werden (Crosby et al. 1993).

Unsere Ergebnisse zeigen ähnliche Werte im Vergleich zur Studie von Crosby et al. 1993. Der mittlere Kraftwert der operierten Hand lag nach 52 Wochen bei $42 \mathrm{~kg}$ x ms (93 lb). In der Studie von Crosby et al. (1993) wurden im Mittel Werte von 45 
$\mathrm{kg} \times \mathrm{ms}(100 \mathrm{lb})$ bis $62 \mathrm{~kg} \times \mathrm{ms}(137 \mathrm{lb})$ erreicht. Allerdings wurde dort eine Aufteilung nach Geschlecht, Hobby, Beruf und dominanter Hand gemacht. 52 Wochen nach operativer Versorgung der Mittelhandfraktur sind fast normale Werte zu messen.

Die Kraftmessungen mit dem JAMARß Hand Dynamometer ergaben Kraftunterschiede von $28 \%$ nach 12 Wochen, $14 \%$ nach 26 Wochen und $7 \%$ nach 52 Wochen, jeweils im Vergleich zur gesunden Gegenseite (siehe 3.5.1). Die Absolutwerte für die operierte Hand sind wenig aussagekräftig (Crosby et al. 1993).

Diagramm 7 zeigt eine Abnahme des Kraftunterschiedes von der 12. bis zur 52. postoperativen Woche. Die Abnahme des Kraftunterschiedes 12 Wochen zu 26 Wochen ist mit $p=0,041(p<0,05)$ signifikant. Die Änderung des Kraftunterschiedes 26 zu 52 Wochen liegt oberhalb des Signifikanzniveaus von $p<0,05$. Insgesamt korreliert der Verlauf des Kraftunterschiedes gemessen mit dem JAMAR® Hand Dynamometer mit fortschreitender Heilung.

Die verbleibende Differenz beruht sehr wahrscheinlich auf dem physiologischen Kraftunterschied zwischen dominanter und nicht - dominanter Hand. Dieser Parameter floss nicht in diese Untersuchung ein, könnte aber bei weiterführenden Studien mit berücksichtigt werden.

\subsubsection{B\&L Engineering PINCH GAUGE ${ }^{\circledR}$}

In der o.g. Studie von Crosby et al. wurden am untersuchten Patientenkollektiv ebenfalls Messungen mit dem B\&L Engineering PINCH GAUGE $®$ durchgeführt. Auch hierbei entstanden vergleichbare Werte zu unseren Studienergebnissen (Crosby et al. 1993).

In unserer Studie liegt der Mittelwert der Kraft nach 52 Wochen beim Schlüsselgriff bei $10 \mathrm{~kg} \mathrm{x} \mathrm{ms} \mathrm{(22} \mathrm{lb),} \mathrm{die} \mathrm{Messungen} \mathrm{von} \mathrm{Crosby} \mathrm{et} \mathrm{al.} \mathrm{ergaben} \mathrm{ähnliche} \mathrm{Werte.}$ Die statistische Auswertung der B\&L Engineering PINCH GAUGE $®$-Messungen bestätigt die Literaturquellen (Crosby et al. 1993). Bei Betrachtung des Kraftunterschieds von operierter zu nicht - operierter Hand liegen die Signifikanzen über dem $\mathrm{p}=0,05$ - Niveau (Diagramm 7).

Bemerkung: Crosby et al. erhoben alle Werte in der Maßeinheit Pfund (lb). Daher wurden die Werte von $\mathrm{kg}$ x ms zu lb umgerechnet. 


\subsubsection{Radiologische Befunde}

\subsubsection{Knöcherne Durchbauung}

Wie bereits unter 3.1.6.1 beschrieben, war eine knöcherne Durchbauung ab der 6 . postoperativen Woche bei 11 von 15 Patienten zu erkennen. Die Beurteilung erfolgte durch einen erfahrenen Unfallchirurgen oder Handchirurgen.

\subsubsection{Kallus und Frakturspaltabgrenzung}

Bei den Patienten mit Kallusbildung im Verlauf muss von einer indirekten Knochenbruchheilung ausgegangen werden (Stürmer 1996). Dies ist jedoch nicht als Nachteil zu verstehen, denn bei Betrachtung der Kraftmessungen, DASH - Score und VAS lässt sich keine Einschränkungen feststellen. Mit einer Kallusbildung ist in den meisten Fällen sogar zu rechnen, da die resorbierbaren Implantate aus Poly L - Lactid deutlich flexibler und elastischer als Metallimplantate sind (Freeland und Jabaley 1998, Freeland und Orbay 2006, Freeland et al. 2001, Klaue et al. 2000). Es kommt zu relevanten Mikrobewegungen innerhalb des Frakturspalts, und damit zu Kallusbildung (Crosby et al. 1993, Stoffel et al. 2000, Stürmer 1996).

\subsection{Ergebnisse - biomechanische Untersuchung}

\subsubsection{Dorsale versus laterale Plattenanlage}

Im Falle einer Operationsindikation sollte die anatomische Wiederherstellung der Achse, Länge und Rotation erreicht und erhalten werden (Massengill et al. 1979, Massengill et al. 1982, Müller et al. 1969). Wie Bozic et al. feststellen, liefert das ideale biodegradierbare Implantat eine relativ konstante Stabilität bis zur knöchernen Konsolidierung (Bozic et al. 2001). Im Verlauf der Knochenbruchheilung übernimmtder Knochen wieder seine Funktion (Bozic et al. 2001, Pietrzak et al. 1996). Mehrere biomechanische Studien belegen, daß die dorsale Plattenanlage die stabilste Versorgung metakarpaler Frakturen darstellt, was die Apex -Belastung von dorsal betrifft (Fini et al. 1995, Fuchs et al. 1998, Low et al. 1995, Massengill et al. 1979, Massengill et al. 1982, van der Elst et al. 1998, van der Elst et al. 2000).

Die laterale Plattenanlage entspricht der von uns gewählten operativen Versorgung der Mittelhandfrakturen. Sie ist besonders im Hinblick auf die anteriore Apex - Belastung stabil (Gautier et al. 2000, Prevel et al. 1995, Prevel et al. 1996). 
Die gemessenen Mittelwerte von $122 \mathrm{~N}$ für die laterale und $133 \mathrm{~N}$ für die dorsale Platte belegen, daß die laterale Plattenanlage keinen nennenswerten Nachteil für die Nachhaltigkeit der operativen Versorgung birgt. Um das Risiko einer sekundären Dislokation möglichst minimieren zu können, bietet sich die unterstützende Schienung mit einer Mittelhandorthese an (Küntscher et

al. 2003, Schleikis 2006). Der Streckapparat mit seinen bradytrophen Eigenschaften kann die einzelnen Bestandteile der biodegradierbaren Implantate nicht gut aufnehmen (Bozic et al. 2001). Durch die Plattenanlage von lateral wird das Risiko einer Fremdkörperreaktion reduziert (Dumont et al. 2007).

Durch die laterale Plattenlage wird die direkte Nachbarschaft zum Extensorapparat vermieden (Bozic et al. 2001). Mechanische Irritationen treten seltener auf. Die Materialentfernung bei resorbierbaren Implantaten ist nicht notwendig (Bozic et al. 2001, Hughes 2006).

Die Kallusbildung beweist, daß relevante Mikrobewegungen im Frakturspalt stattfinden (Dumont et al. 2007, Stürmer 1996). Dies hat jedoch keinen Einfluss auf die Retention der Reposition (Klaue et al. 2000, Stürmer 1996).

\section{Zusammenfassung}

Mittelhandfrakturen sind häufige Verletzungen der oberen Extremität, die besonders junge Menschen betreffen (Abdon et al. 1984, Crosby et al. 1994, Prevel et al. 1995, Prevel et al. 1996, Prokop et al. 1999, Prokop et al. 2002, Schaefer und Siebert 2000). Etwa 10\% aller Frakturen sind Mittelhand- oder Fingerfrakturen (Barton 1984, De Jonge et al. 1994, Schaefer und Siebert 2000). Da bereits geringe Verkürzungen, Achs- und Rotationsabweichungen der Mittelhandknochen nach Fraktur deutliche Auswirkungen auf die Handfunktion haben, ist bei der Frakturbehandlung auf die Sicherstellung der Länge, Achse und Rotation der Mittelhandknochen zu achten (Birndorf et al. 1997, Low et al. 1995).

Es ist unbestritten, daß eine konservative Behandlung von extraartikulären, geschlossenen, nicht oder nur gering dislozierten Mittelhandfrakturen prinzipiell möglich ist (Küntscher et al. 2003, Schaefer und Siebert 2000, Tavassoli et al. 2005, Trevisan et al. 2004). Diese Frakturen stellen den überwiegenden Anteil der Mittelhandfrakturen dar (Barton 1984, Feehan und Bassett 2004, Freeland und Jabaley 1998, Freeland und Orbay 2006, Freeland et al. 2001). Sie können sicher und ef- 
fektiv mit Schienungsverfahren (Buddy - Tape, Mittelhandorthese, Gips) behandelt werden (Bain und Guy 2003, Freeland und Jabaley 1998, Freeland und Orbay 2006, Freeland et al. 2001, Schleikis 2006, Trevisan et al. 2004). Bei primärer oder sekundärer Dislokation, multiplen frakturierten Strahlen, offenen oder pathologischen Frakturen ist eine operative Behandlung indiziert (Freeland und Jabaley 1998, Freeland und Orbay 2006, Freeland et al. 2001, Schmidt und Lanz 1992).

Die Plattenosteosynthese im Rahmen der offenen operativen Verfahren liefert exzellente Stabilität im Frakturbereich (Eppley und Sadove 1995).

Beim Einsatz resorbierbarer Implantate stellt die Plattenanlage von lateral keinen Nachteil gegenüber einer dorsalen Anlage dar (Fini et al. 1995, Fuchs et al. 1998, Litsky 1993, Massengill et al. 1979, Massengill et al. 1982, van der Elst et al. 1998, van der Elst et al. 2000). Resorbierbare Implantate sind stabil und widerstandsfähig genug, ohne die Eigenschaften von Metallimplantaten zu erreichen (Fini et al. 1995, Fitoussi et al. 1998, Prevel et al. 1995, Prevel et al. 1996, Waris et al. 2002, Waris et al. 2004). Somit liefern resorbierbare Implantate die guten mechanischen Eigenschaften der Plattenosteosynthese. Der Folgeeingriff zur Implantatentfernung entfällt (Bozic et al. 2001, Eppley und Sadove 1995).

Durch die Plattenanlage von lateral wird das Auftreten einer unspezifischen Fremdkörperreaktion deutlich reduziert, die frühfunktionelle Nachbehandlung kann ungestört erfolgen. Allerdings kann durch den operativen Zugang eine überschießende Bindegewebsbildung während des Heilungsprozesses hervorgerufen werden, die ihrer-seits funktionelle Defizite hinterlassen kann (Duncan et al. 1993, Freeland und Jabaley 1998, Freeland und Orbay 2006, Freeland et al. 2001).

Es gilt in Zukunft zu untersuchen, ob die sekundären Komplikationen wie Refraktur, Bildung von sterilen Abszessen und fibrösen Abkapselungen nicht problematischer sind als Plattenirritation, „stress shielding“ und Sehnenadhäsion (Hughes 2006, Klaue et al. 2000, Stoffel et al. 2000). 


\section{Anhang}

\subsection{Jahss Manöver}

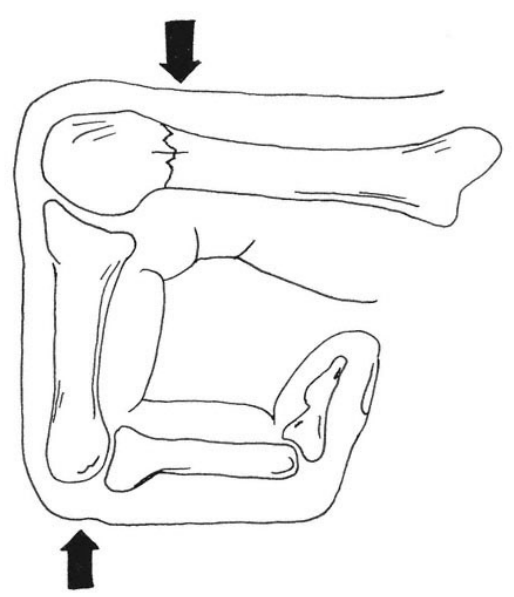

Abbildung 22: Repositionstechnik

\subsection{Visuelle Analogskala (VAS)}

\section{(-) Visuelle Analogskala (VAS)}

kein Schmerz

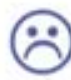

stärkster Schmerz

Abbildung 23: VAS 


\subsection{DASH - Score}

\section{Disabilities of the arm, shoulder and hand (DASH) (Germann et al 1999)}

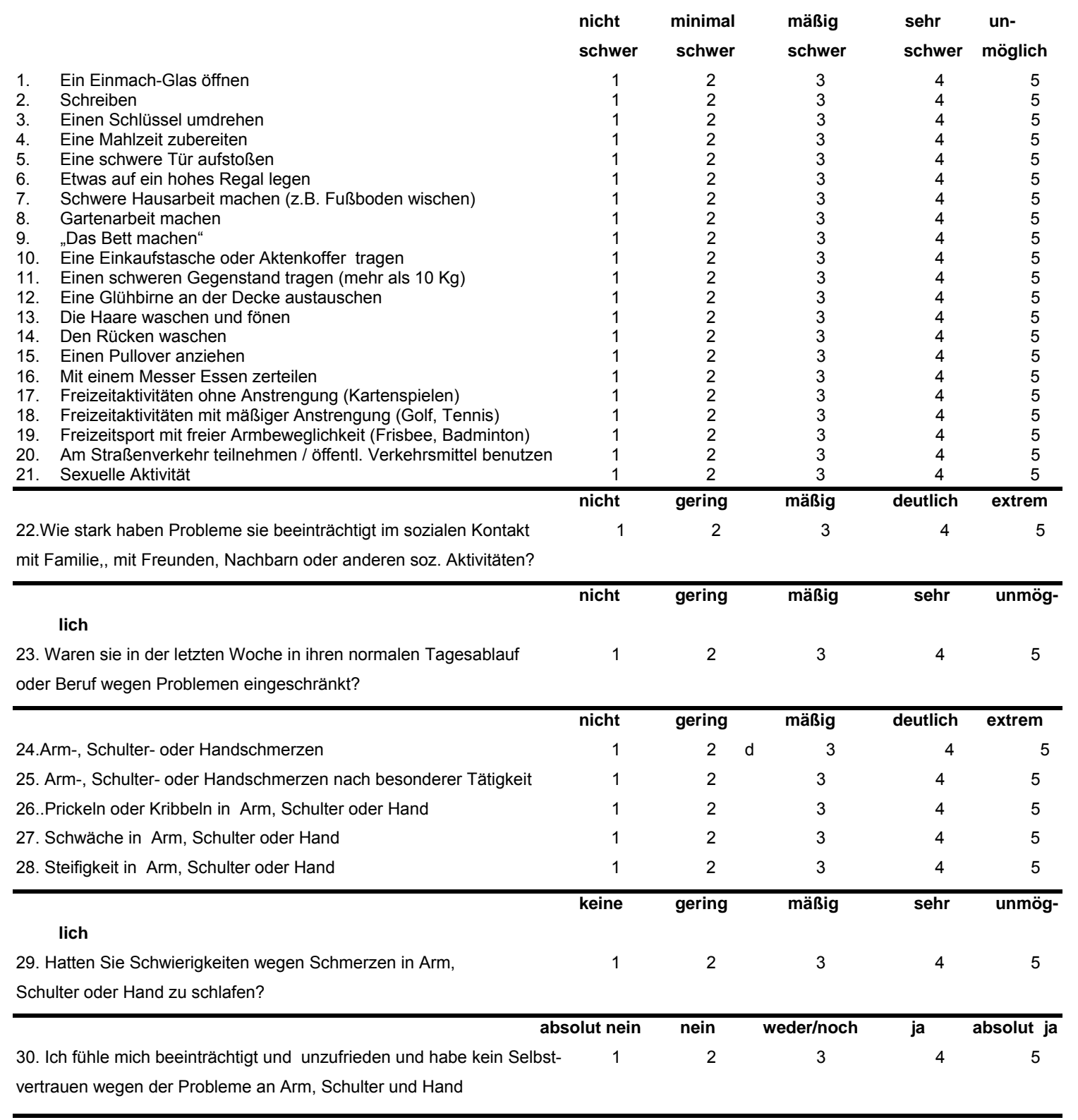




\subsection{Fallvorstellung - klinisch und radiologisch}

Bereits die erste klinische Nachuntersuchung des Patienten war sehr zufrieden stellend. Im Verlauf traten keine Bewegungseinsschränkungen auf. Exemplarisch wird eine Funktionsprüfung vorgestellt.
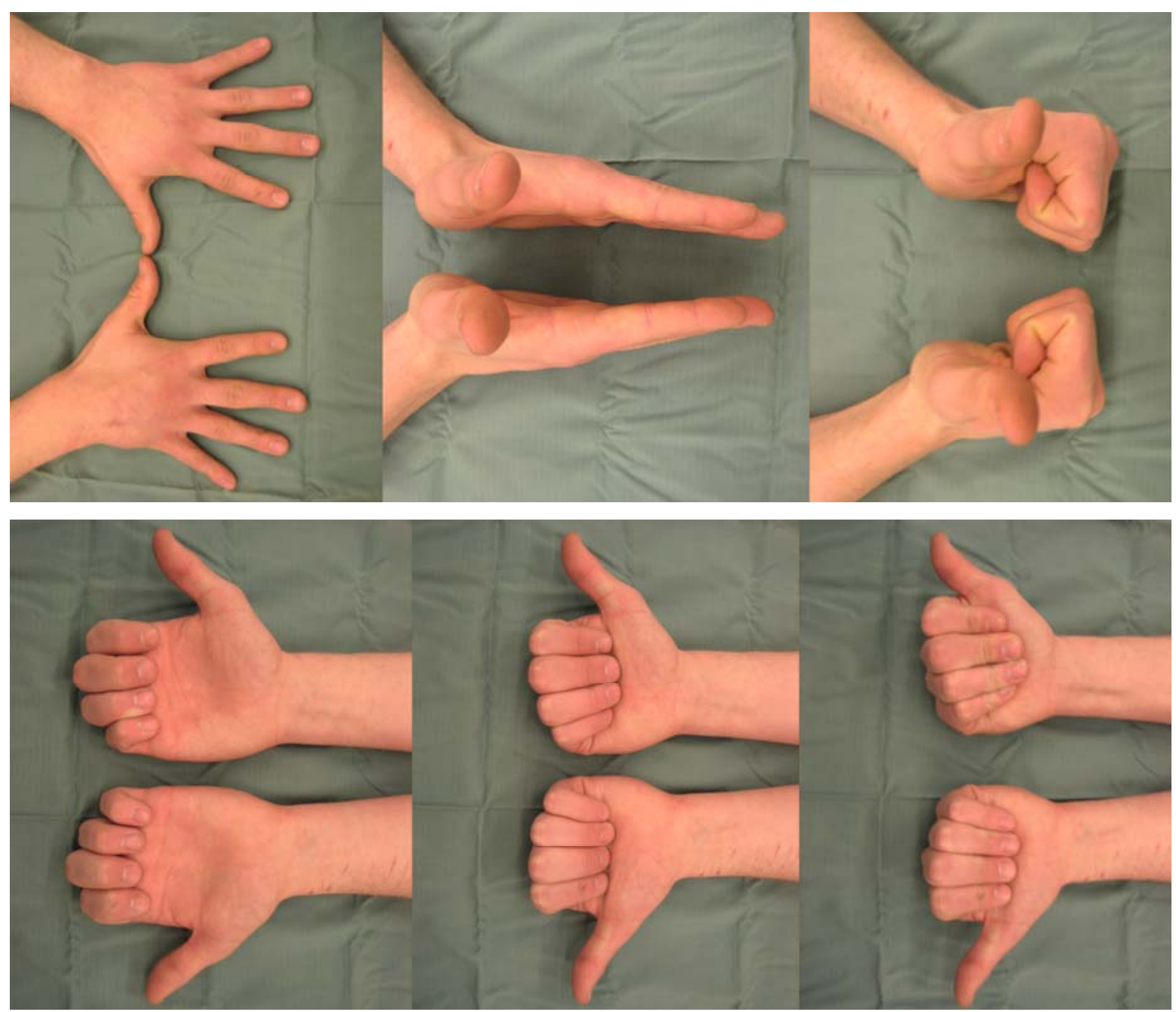

Abbildung 24: klinischer Untersuchungsgang bei einem Patienten mit Fraktur der MHK III und IV rechts. Beachten Sie den MHK V der rechten Hand mit Rotationsfehler nach konservativer Therapie. 


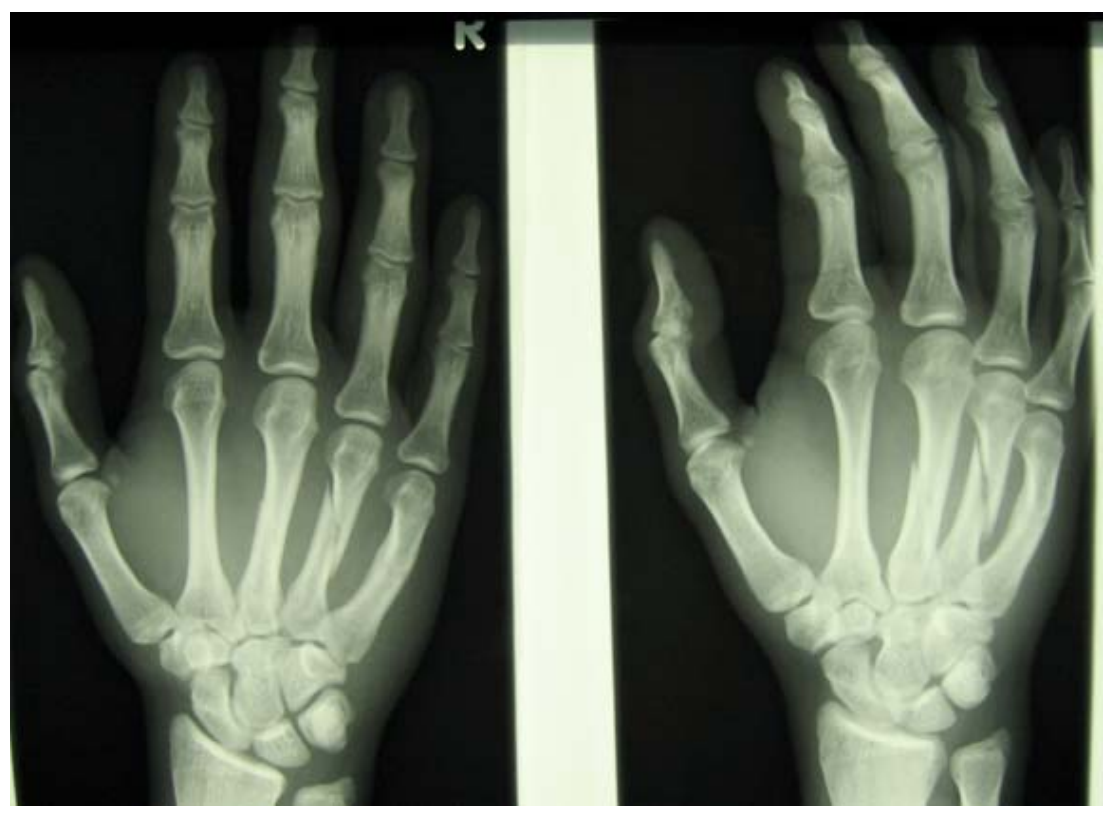

Abbildung 25: Unfallaufnahmen in 2 Ebenen mit dislozierter Fraktur der MHK III und IV, sowie verheilter Fraktur des MHK V rechts

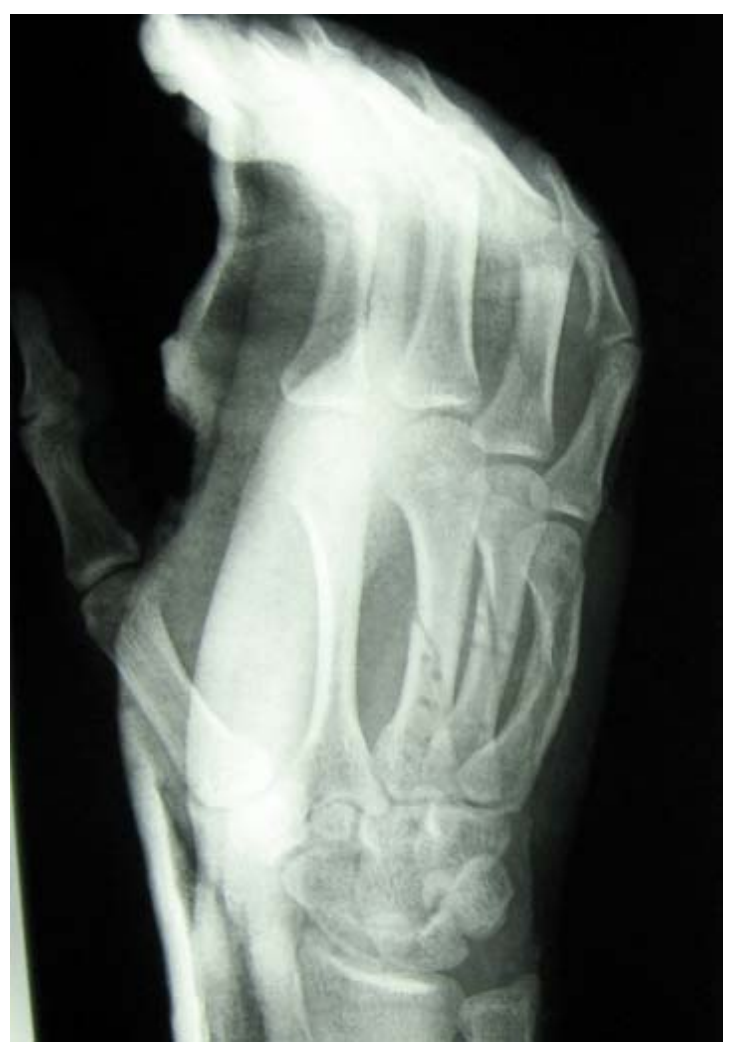

Abbildung 26: Röntgenkontrolle 2 Wochen postoperativ 

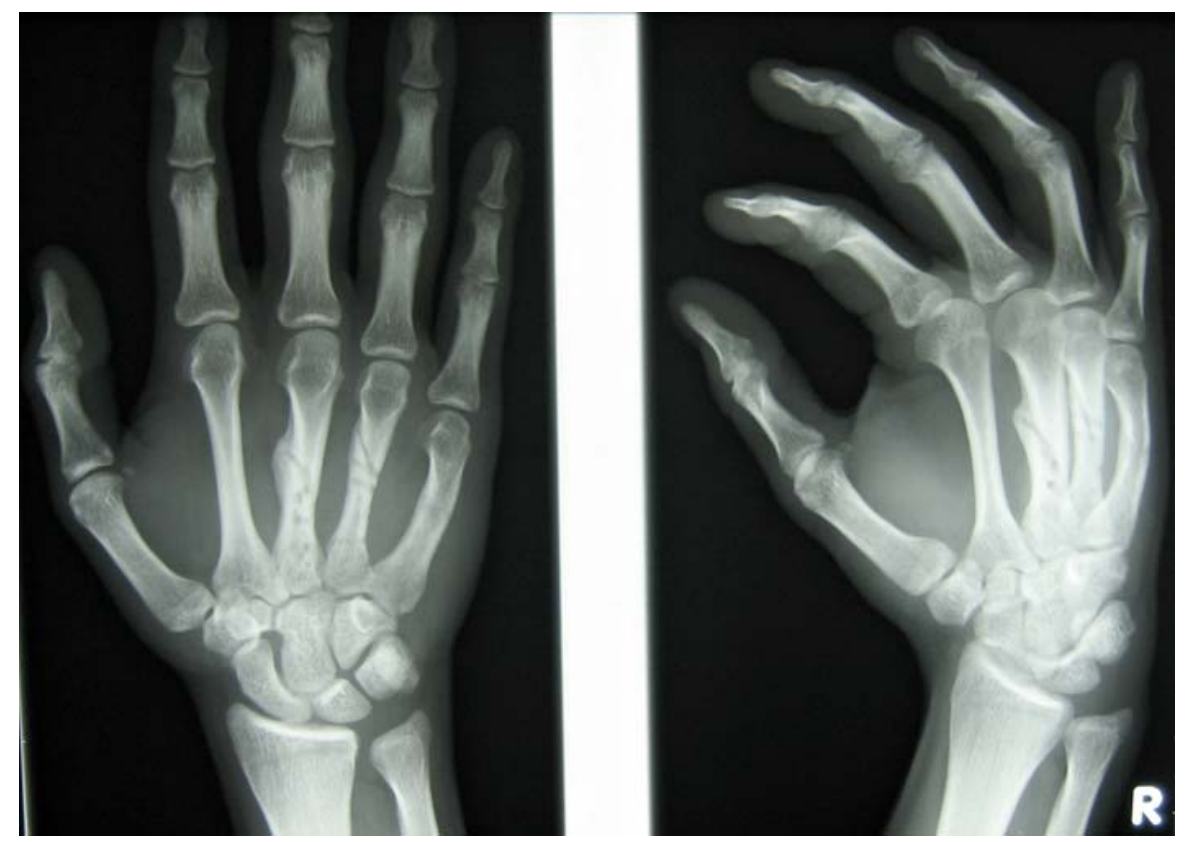

Abbildung 27: Röntgenkontrolle 6 Wochen postoperativ
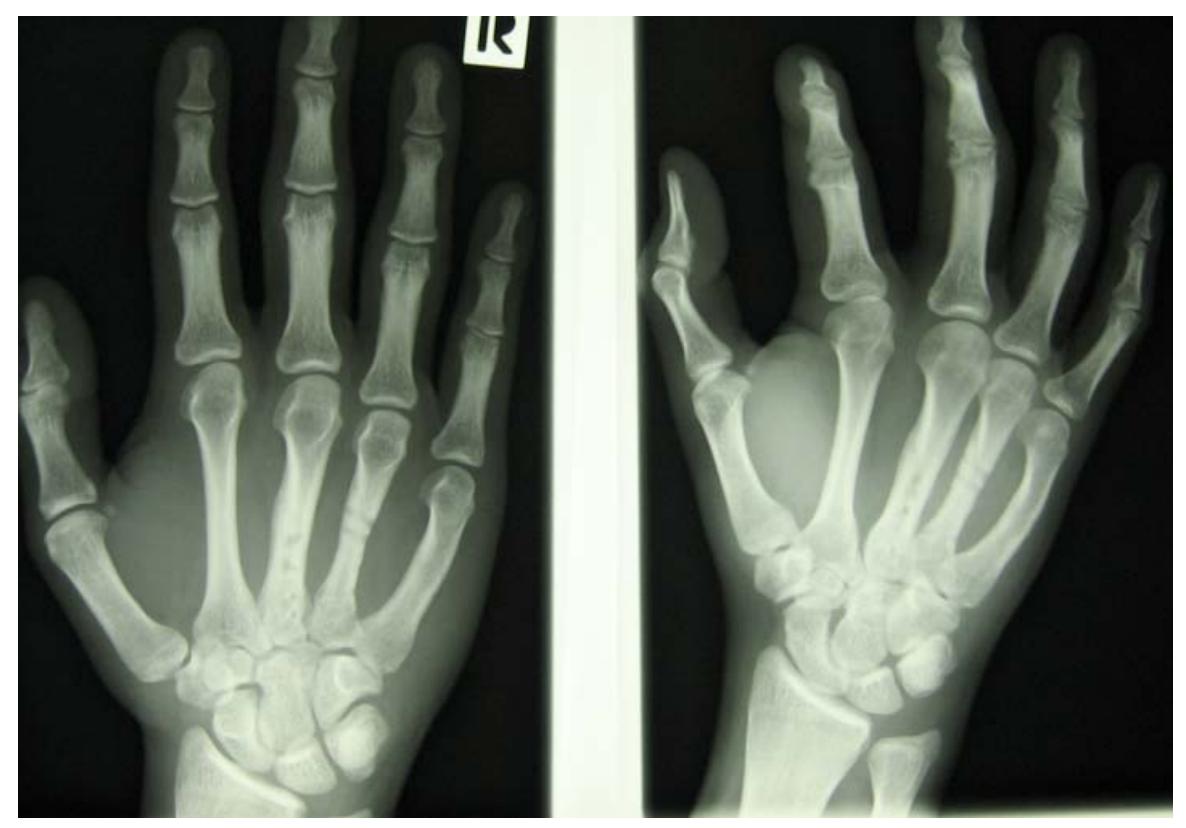

Abbildung 28: Röntgenkontrolle 26 Wochen postoperativ 


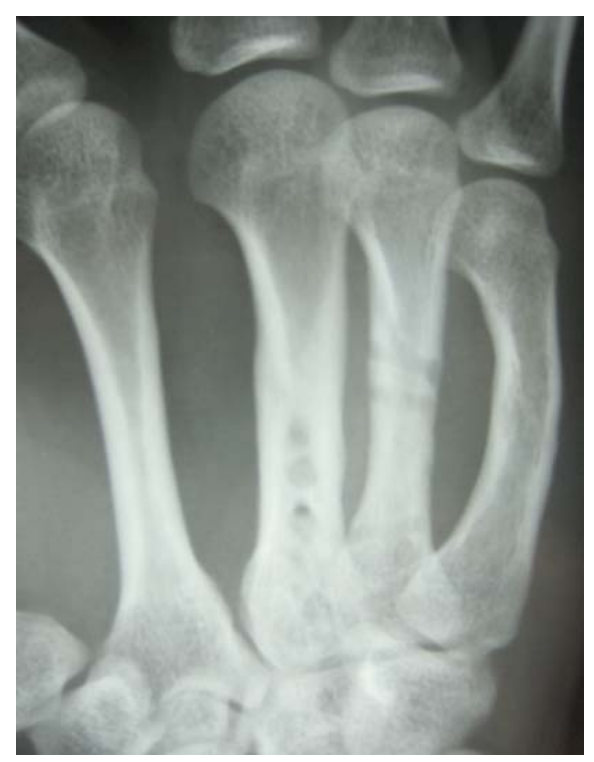

Abbildung 29: Abschlussröntgen 52 Wochen postoperativ

\section{Beurteilung:}

Insgesamt komplikationsloser Verlauf sowohl klinisch als auch radiologisch. Die beiden osteosynthetisch versorgten MHK III und IV verheilen ohne Längenminderung und Rotationsabweichung. 


\section{Literaturverzeichnis}

Abdon P, Mühlow A, Stigsson L (1984): Subcapital fractures of the fifth metacarpal bone. Arch Orthop Trauma Surg, 103,231-234

Aleksandrowicz R, Pagowksi S, Seyfried A: Anatomic - geometric and kinematic analysis of the metacarpo - phalangeal articulation of the third digit of the human hand. Fol Morphol 33 (1974) 353-361

Arbeitskreis "bildgebende Diagnostik in der Rheumatologie" des Regionalen Rheumazentrums Berlin e.V. (1997): Empfehlungen zur Röntgendiagnostk der Hände und Füße bei rheumatologischen Fragestellungen. Berliner Ärztebl 110, $771-772$

Bain GI, Guy SD (2003): Derotation taping for metacarpal and phalangeal fractures. Tech Hand Up Extrem Surg 7, 130 - 132

Barton NJ (1984): Fractures of the hand. J Bone Joint Surg 66, 159 - 167

Bechtol CO (1954): Grip test: Use of dynamometer with adjustable handle spacings. J Bone Joint Surg 36, 820 - 824, 832

Becker HJ, Wiltfang J, Merten HA, Luhr HG (1999): Biodegradierbare Miniplatten (LactoSorb ) bei Kranioosteoplastik - experimentelle Ergebnisse am schnell wachsenden Miniaturschwein. Mund Kiefer Gesichtschir 3, 275-278

Bergsma JE, De Bruijn WC, Rozema FR, Bos RRM, Boering G (1995): Late degradation tissue response to poly (L-lactide) bone plates and screws. Biomaterials $16,25-31$

Birndorf MS, Daley R, Greenwald DP (1997): Metacarpal fracture angulation decreases flexor mechanical efficiency in human hands. Plast Reconstr Surg 99, $1079-1083$

Bozic KJ, Perez LE, Wilson DR, Fitzgibbons PG, Jupiter JB (2001): Mechanical testing of bioresorbable implants for use in metacarpal fracture fixation. $\mathrm{J}$ Hand Surg 26A, 755-761

Böstmann O, Hirvensalo E, Mäkinen J, Rokkanen P (1990): Foreign-body reactions to fracture fixation of biodegradable synthetic polymers. J Bone Joint Surg

(Br) 72-B , $592-596$

Brewerton DA (1967): A tangential radiographic projection fot demonstrating involvement of metacarpal heads in rheumatic arthritis. Br J Radiol 40, 233

Brüser P, Krein R, Larkin G (1999): Fixation of metacarpal fractures using absorbable hemi-cerclage sutures. J Hand Surg (Br, Eur) 24-B, 6, 683-687

Crosby CA, Wehbe MA, Mawr B (1993): Hand strength: normative values. J Hand Surg (A) 19-A, 665 - 670

De Jonge JJ, Kingma J, Van Der Lei B, Klasen HJ (1994): Fractures of the 
metacarpals. A retroperspective analysis of the incidence and aetiology and a review of the English-language literature. Injury 25, 365 - 369

Dubousset JF: The digital joints. In: The Hand. Hrsg. Tubiana R. Vol. 1. Saunders WB, Philadelphia 1981

Dumont C, Fuchs M, Burchhardt H, Bohr S, Appelt D, Stürmer KM (2007): Clinical results of absorbable plates for displaced metacarpal fractures. J Hand Surg 32A, $491-496$

Duncan RW, Freeland AE, Jabaley ME, Meydrech EF (1993): Open hand fractures. An analysis of the recovery of active motion and complications. J Hand Surg 18, 387 - 394

Eppley BL, Sadove AM (1995): A comparison of resorbable and metallic fixation healing of calvarial bone grafts. Plast Reconstr Surg 96, 316 - 322

Feehan LM, Bassett K (2004): Is there evidence for early mobilization following extra - articular hand fracture? J Hand Ther 17, 300 - 308

Fess EE, Moran C (1981): Clinical assessment recommendations. American Society of Hand Therapists, Monograph, Indianapolis

Fini M, Gianni S, Giardino R, Giavaresi G, Grimladi M (1995): Resorbable device for fracture fixation: in vivo degradation and mechanical behaviour. J Artif Organs $18,772-776$

Fitoussi F, Lu W, Ip WY, Chow SP. (1998): Biomechanical properties of absorbable implants in finger fractures. J Hand Surg 23B, $79-83$

Freeland AE, Jabaley ME: Open reduction internal fixation: metacarpal farctures. In: Strickland JW, editor. The Hand. Master Techniques In Orthopaedic Surgery. Lippincott-Raven, Philadelphia 1998: 3 - 33

Freeland AE, Orbay JL (2006): Extraarticular hand fractures in adults. Clin Orthop Rel Res. 445,133 - 145

Freeland AE, Geissler W, Weiss AP (2001): Operative treatment of common displaced and unstable fractures of the hand. J Bone Joint Surg (A) 83A, 6, 928 - 945

Frohse F, Fränkel M: Die Muskeln des menschlichen Armes. In: von Bardelebens K: Handbuch der Anatomie des Menschen. Bd. 2, Abt. 2, Teil 2. G. Fischer, Jena 1908

Fuchs M, Vosshenrich R, Dumont C, Stürmer KM (2003): Refixation osteochondraler Fragmente mit resorbierbaren Implantaten. Chirurg 74, 554 - 561

Fuchs M, Köster G, Krause T, Merten HA, Schmid A (1998): Degradation of and interosseous reactions to bioderagdable poly-L-lactide screws. Arch Orthop Trauma Surg 118, 140 - 144 
Gautier E, Perren SM, Cordey J (2000): Effect on plate position relative to bending direction on the rigidity of a plate osteosynthesis. A theoretical analysis. Injury 31 , S-C14-20

Germann G, Wind G, Harth A (1999): Der DASH - Fragebogen - Ein neues Instrument zur Beurteilung von Behandlungsergebnissen an der oberen Extremität. Handchir Mikrochir Plast Chir 31, 149 - 152

Guillermo, Dillard F, Clelland N, Rashid R (2005): Comparison of strains produced by titanium and poly $\mathrm{D}$, L-lactide acid plating systems to in vitro forces. $\mathrm{J}$

Oral Maxillofac Surg 63, 968 - 972

Haerle M, Schmidt G, Schaller HE (2002): Messung von Bewegungsumfängen, Sensibilität, Volumen, Kraft und Funktion der Hand. Akt Traumatol 32, 129 - 136

Hanemann jr M, Simmons O, Jain S, Baratta R, Guerra AB, Metzinger SE (2005): A Comparison of Combinations of Titanium and Resorbable Plating Systems for Repair of Isolated Zygomatic Fractures in the Adult. Ann Plast Surg $54,402-408$

Heidemann W, Fischer JH, Koebke J, Bussmann C, Gerlach KL (2003): In-vivoUntersuchung zur Degradation von Poly-Laktid und Poly-Lactid-co-GlykolidOsteosynthesematerial. Mund Kiefer Gesichtschir 7, 283 - 288

Hofmann GO (1992) Biodegradable implants in orthopaedic surgery. Clin Mat $10,75-80$

Hofmann GO (1997): Biologisch abbaubare Knochenimplantate. Spektr Wiss, $46-50$

Hofmann GO, Wagner FD (1993): New Implant designs for bioresorbable devices in orthopaedic surgery. Clin Mat 14, 207 - 215

Hofmann GO, Liedtke H, Ruckdeschel G, Lob G (1990): The influence of bacterial contaminations on the biodegradation of PLA implants. Clin Mat 6, 137 - 150

Hofmann GO, Wagner FD, Keller A, Feist H (1996): Postoperative irradiation treatment and bioresorbable implants in orthopaedic surgery. Biomaterials (B) 17B, $1149-1153$

Hughes TB (2006): Bioabsorbable implants in the treatment of hand fractures. Clin Orthop Rel Res 445, 169 - 174

Jester A, Harth A, Wind G, Germann G, Sauerbier M (2005): Ersetzt der Disabbility of Arm, Shoulder and Hand Questionnaire (DASH - Fragebogen) die Erfassung von Bewegungsausmaß und Kraft bei der Bewertung von Ergebnissen? Handchir Mikrochir Plast Chir 37, 126 - 130

Kapandji IA: Anatomie fontionelle. In: Tubiana R: Traité de chirurgie de la main. 
Masson, Paris 1980, 55 - 90

Klaue K, Fengels I, Perren SM (2000): Long-term effects of plate osteosynthesis: Comparison of four different plates. Injury 31, S-B 51-62

Kulkarni RK, Pani KC, Neumann C (1966): Polylactic acid for surgical implants. Arch Surg 93, 839 - 843

Kujala S, Raatikainen T, Kaarela O, Ashammakhi N, Ryhänen J (2004): Succesful treatment of scaphoid fractures and nonunions using bioabsorbable screws. J Hand Surg (A) 29A, 68 - 73

Kunz E, Weckbach A, Rein S (1995): Resorbierbare Osteosynthesestifte. Unfallchirurgie $21,1-7$

Küntscher MV, Schäfer DJ, Germann G, Siebert HR (2003): Frakturen der Mittelhand. Chirurg 74, 1018 - 1025

Koebke J, Stümpel E: Untersuchungen zu einer Funktionsanalyse der Metacarpophalangealgelenke II - IV der menschlichen Hand. Verh Anat Ges 75 (1981) 275-276

Larkin G, Brüser P, Safi A (1997): Die Möglichkeiten und Grenzen der intramedullären Kirschner-Drahtosteosynthese zur Behandlung der Metakarpalfrakturen.

Handchir Mikrochir Plast Chir 29, 192 - 196

Landsmeer, JMF (1955): Anatomical and functional inverstigations on the articulations of the human fingers. Acta Anatom 25 (Supplement 24), 1-69

Lazar G, Schulter - Ellis FP (1980): Intramedullary structure of human metacarpals. J Hand Surg 5 (5), 477 - 481

Leenslag JW, Pennings AJ, Bos RRM, Rozema FR, Boering G (1987): Resorbable materials of poly (L-lactide). VI. Plates and screws for internal fracture fixation. Biomaterials $8,70-73$

Litsky AS (1993): Bioabsorbable Implants for Orthopaedic Fracture Fixation. J Appl Biomat 4, $109-111$

Lourie GM, Gaston RG, Freeland AE (2006): Collateral ligament injuries of the metacarpophalangeal joints of the fingers. Hand Clin 22, 357-364

Low CK, Wong HC, Low YP, Wong HP (1995): A cadaver study of the effects of dorsal angulation and shortening of the metacarpal shaft on the extension and flexion force ratios of the index and little fingers. J Hand Surg (Br) 20, 609 - 613

Massengill JB, Alexander H, Parson JR, Schecter MJ (1979): Mechanical analysis of Kirschner wire fixation in a phalangeal model. J Hand Surg 4-4, 351 $-356$

Massengill JB, Alexander H, Langrana N, Mylod A (1982): A phalangeal fracture 
model - quantitative analysis of rigidity and failure. J Hand Surg 7-3, 264 - 270

Mathiowetz V, Kashmann N, Volland G, Weber K, Dowe M, Rogers S (1985): Grip and pinch strength: normative values for adults. Arch Phys Med Rehabil $66,69-72$

Mollaoglu N, Cetiner S, Alpaslan C, Gültekin SE, Alpar R (2003): The early tissue response to titanium and LactoSorb screws. Dent Traumatol Denmark 19, $139-144$

Müller ME, Allgöwer M, Willenegger H: Manual der Osteosynthese. AO - Technik 1. Auflage, Springer Verlag, Berlin, Heidelberg, New York, 1969

Navarro M, Michiardi A, Castaño O, Planell JA (2008): Biomaterials in orthopaedics. J Interfac 5, 1137 - 1158

Nunley JA II, Joneschild E (2006): Metacarpal Fractures. Orthopaedic Care Online Textbook (www.orthopaediccare.net), 1 - 11

Pechlaner S, Kerschbaumer F, Hussl H: Operationsatlas Handchirurgie, Thieme Verlag Stuttgart, 1998, 310

Peter Brehm GmbH (2008): www.peter-brehm.de

Petracic B, Siebert H (1998): AO-Klassifikation der Frakturen des Handskeletts. Handchir Mikrochir Plast Chir 30, 40 - 44

Pietrzak WS, Sarver D, Verstynen M (1996): Bioresorbable implants. Bone 19, 109-119

Pietrzak WS, Sarver D, Verstynen M (1997): Bioabsorbable Polymer Science for the practicing surgeon. J Craniofac Surg 8, 87 - 91

Prevel CD, Eppley BL, Jackson JR, Moore K, McCarty M, Sood R, Wood R [corrected to Sood R] (1995): Mini and Micro Plating of Phalangeal and Metacarpal Fractures: A biomechanical study. J Hand Surg 20A, 44 - 49

Prevel CD, Morgan R, Molnar J, Eppley BL, Moore K (1996): Biomechanical Testing of Titanium Self-Tapping versus pretapped lag screw fixation of spiral metacarpal fractures. Ann Plast Surg 37, 34 - 40

Prokop A, Jubel A, Helling HJ, Kulus S, Rehm KE (2002): Behandlungsmaßnahmen bei Mittelhandfrakturen. Handchir Mikrochir Plast Chir 34, 328 - 331

Prokop A, Kulus S, Helling HJ, Burger C, Rehm KE (1999): Gibt es Richtlinien zur Behandlung von Mittelhandfrakturen? Unfallchirurg 1999; 102, 50 - 58

Rösler J, Harders H, Bäker M: Mechanisches Verhalten der Werkstoffe. GWV Fachverlage $\mathrm{GmbH}$, 3., durchgesehene und korrigierte Auflage, Springer Verlag, $2008,39-43$

Roos E, Maile K: Werkstoffkunde für Ingenieure. 3., neu bearbeitete Auflage 
Springer Verlag, 2008, 72-76

Royle SG (1990): Rotational deformity following metacarpal fracture. J Hand Surg (BR) 15B, 124-125

Schaefer M, Siebert HR (2000): Finger - und Mittelhandfrakturen. Operative und nicht operative Behandlungsverfahren, Teil 1 und Teil 2, Unfallchirurg 103, 482 - 494, 582 - 592

Schleikis A: Gips und synthetischer Stützverband. Steinkopff Darmstadt, 2006, $76-79$

Schmidt HM, Lanz U: Chirurgische Anatomie der Hand, Hippokrates Verlag, Stuttgart, 1992, 138 - 152

Scott J, Huskisson EC (1976): Graphic representation of pain. Pain 2, 175 - 184

Shyam Kumar AJ, Parmar V, Ahmed S, Kar S, Harper WM (2008): A study of grip endurance and strength in different elbow positions. J Orthopaed Traumatol $9,209-211$

Stoffel K, Klaue K, Perren SM (2000): Functional load of plates in fracture fixation in vivo and its correlate in bone healing. Injury 31 , S-B $37-50$

Strauch RJ, Rosenwasser MP, Lunt JG (1998): Metacarpal shaft fractures: the effect of shortening on the extensor tendon mechanism. J Hand Surg (A) 23A, $519-523$

Stürmer KM (1996): Die elastische Plattenosteosynthese, ihre Biomechanik, Ihre indikation und Technik im Vergleich zur rigiden Osteosynthese. Unfallchirurg $99,816-829$

Taeger G, Podleska LE, Ruchholtz S, Sommerfeldt D, Nast - Kolb D (2007): Epiphysennahe Frakturen bei Kindern, Teil I: Obere Extremität. Unfallchirurg. 110, $867-883$

Tavassoli J, Ruland RT, Hogan CJ, Cannon DL (2005): Three cast techniques for the treatment of extra-articular metacarpal fractures. Comparison of shortterm outcome and final fracture alignement. J Bone Joint Surg 87 (10), 2196 2201

Trevisan C, Moraganti A, Casiraghi A, Marinoni EC (2004): Low-severity metacarpal and phalangeal fractures treated with miniature plates and screws. Arch Orthop Trauma Surg 124(10), 675 - 678

Van der Elst M (2000): Biologisch abbaubare Implantate in der Unfallchirurgie. Unfallchirurg 103, 177

Van der Elst M, Bramer J, Klein C (1998): Biodegradable interlocking nails for fracture fixation. Clin Orth Rel Res 357, 192 - 204

Van der Elst M, Patka P, Van Der Werken C, (2000): Resorbierbare Implantate 
für Frakturfixierungen. Unfallchirurg 103, 178 - 182

Waris E, Ashammakhi N, Raatikainen T, Törmälä P, Santavirta S, Konttinen Y (2002): Self-reinforced bioabsorbable versus metallic fixation systems for metacarpal and phalangeal fractures. J Hand Surg (A) 27A, 902 - 908

Waris E, Ninkovic M, Harpf C, Ashammakhi N (2004): Self-reinforced bioabsorbable miniplates for skeleton fixation in complex hand surgery. J Hand Surg (A) 29A, $452-457$

Weiler A, Helling HJ, Kirch U, Zirbes TK, Rehm K (1996): Foreign-body reaction and the course of osteolysis after polygycocolide implants for fracture fixation. $\mathrm{J}$ Bone Joint Surg (Br) 78B, $369-376$ 


\section{Abkürzungsverzeichnis}
ATP Adenosintriphosphat
CT Computertomographie
DASH Disabilities of the Arm, Shoulder and Hand
MCP Metakarpophalangealgelenk
MHK Mittelhandknochen
MRT Magnetresonanztomographie
VAS Visuelle Analogskala 


\section{Danksagung}

Herzlicher Dank gebührt Herrn Privatdozent Dr. med. Clemens Dumont, Oberarzt der Abteilung Unfallchirurgie, Plastische und Wiederherstellungschirurgie des Universitätsklinikums Göttingen, für die ausdauernde Unterstützung bei der Erstellung der Dissertation. Trotz seines eng bemessenen Terminkalenders nahm er sich Zeit, um Lösungsvorschläge zu unterbreiten.

Danken möchte ich Herrn Michael Heier, Fa. Biomet - Merck®, für die Bereitstellung der resorbierbaren Implantate zur Durchführung der biomechanischen Versuche.

Herrn Adolf Schleikis, Poliklinik der Abteilung Unfallchirurgie, Plastische und Wiederherstellungschirurgie des Universitätsklinikums Göttingen, danke ich herzlich für die Unterstützung bei der Erstellung der Fotografien.

Frau Claudia Stausberg danke ich herzlich für die professionelle Nachbearbeitung der Fotos. 


\section{Lebenslauf}

Am 04. Mai 1980 wurde ich, Daniel Uwe Mario Appelt, als erster Sohn des Berufsoffiziers Reinhard Appelt und der Industriekauffrau Doris Appelt, geborene Zilger, in München geboren. 1986 wurde ich in die erste Klasse der Grundschule in Boxberg bei Bad Mergentheim eingeschult, nachdem ein Umzug der Familie aus dienstlichen Gründen meines Vaters dorthin erfolgt war. Aufgrund einer weiteren Versetzung meines Vaters wechselte ich 1989 in die 4. Klasse der Grundschule in Hannover - Bemerode. Anschließend besuchte ich die 5. und 6. Klasse der Orientierungsstufe und ab 1993 für vier Jahre das Bismarck - Gymnasium in Hannover. Nach Abschluss der 10. Klasse nahm ich 1996 an einem einjährigen Schüleraustausch des Rotary - Club Hannover teil. In Cornwall, Ontario/Kanada besuchte ich die High School. Im Sommer 1997 kehrte ich nach Deutschland zurück, um kurz darauf von Hannover nach Bendorf bei Koblenz umzuziehen. Dort schloss ich 2000 am Wilhelm - Remy - Gymnasium meine Schulausbildung mit dem Abitur ab. Im Juli 2000 trat ich in die Bundeswehr ein. Dort absolvierte ich die allgemeine Grundausbildung sowie die sanitätsdienstliche Ausbildung. Im Januar 2001 erfolgte die Übernahme in die Laufbahn „Sanitätsoffizieranwärter der Bundeswehr." Es folgten mehrere militärische und sanitätsdienstliche Lehrgänge in Montabaur und München. Nach meiner Versetzung nach Göttingen begann ich im Herbst 2001 das Studium der Humanmedizin an der Georg - August - Universität. Zeitgerecht absolvierte ich im Sommer 2003 das Physikum und trat im Sommer 2006 mein praktisches Jahr an. Das praktische Jahr verbrachte ich zu jeweils 4 Monaten in Goslar (Innere Medizin), Basel (Orthopädie) und in Göttingen (Allgemeinchirurgie). Im Dezember 2007 schloss ich das Studium erfolgreich mit der Approbation als Arzt ab. Nach der Versetzung an das Bundeswehrzentralkrankenhaus Koblenz trat ich im Dezember 2007 meinen Dienst als Assistenzarzt der Neurochirurgie an. AnschlieBend wurde ich im Juli 2008 in die Abteilung Orthopädie und Unfallchirurgie übernommen. Im Januar 2009 trat ich aus der Bundeswehr aus und nahm eine Stelle als akademischer Rat des Universitätsklinikums Essen, Abteilung Unfallchirurgie an. Dort bin ich als Assistenzarzt in der Unfallchirurgie beschäftigt. 